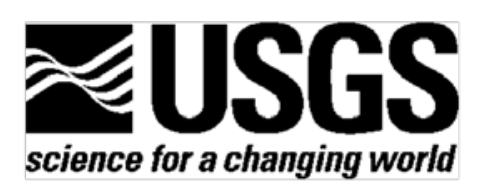

\title{
Should Ground-Motion Records be Rotated to Fault-Normal/Parallel or Maximum Direction for Response History Analysis of Buildings?
}

By Juan C. Reyes and Erol Kalkan

Open-File Report 2012-1261

U.S. Department of the Interior

U.S. Geological Survey 


\section{U.S. Department of the Interior \\ KEN SALAZAR, Secretary}

\section{U.S. Geological Survey \\ Marcia K. McNutt, Director}

U.S. Geological Survey, Reston, Virginia: 2012

For product and ordering information:

World Wide Web: http://www.usgs.gov/pubprod/

Telephone: 1-888-ASK-USGS

For more information on the USGS-the Federal source for science about the Earth, its natural and living resources, natural hazards, and the environment:

World Wide Web: http://www.usgs.gov/

Telephone: 1-888-ASK-USGS

Suggested citation: Reyes, J.C., and Kalkan, E., 2012, Should ground-motion records be rotated to fault-normal/parallel or maximum direction for response history analysis of buildings?: U.S. Geological Survey Open-File Report 2012-1261, 89 p.

Any use of trade, product, or firm names is for descriptive purposes only and does not imply endorsement by the U.S. Government.

Although this report is in the public domain, permission must be secured from the individual copyright owners to reproduce any copyrighted material contained within this report. 


\section{Contents}

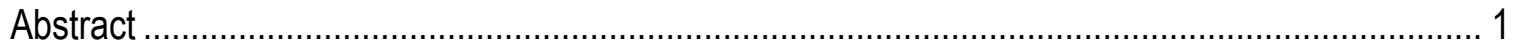

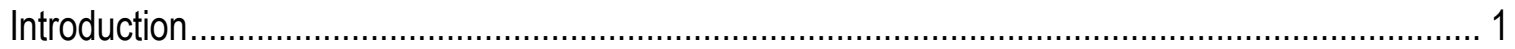

Ground Motions Selected .......................................................................................... 3

Description of Structural Systems and Computer Models .......................................................... 6

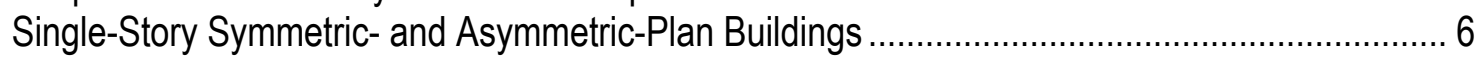

Multistory Symmetric- and Asymmetric-Plan Buildings ………….................................... 7

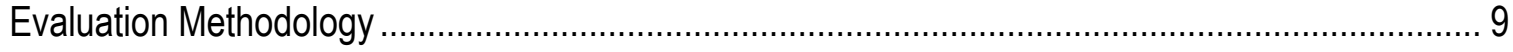

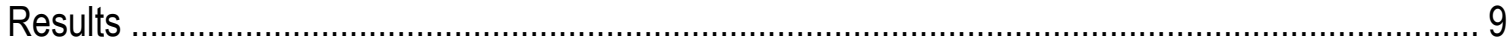

Single-Story Symmetric- and Asymmetric-Plan Buildings ………………………….......... 9

Multistory Symmetric- and Asymmetric-Plan Buildings .................................................... 11

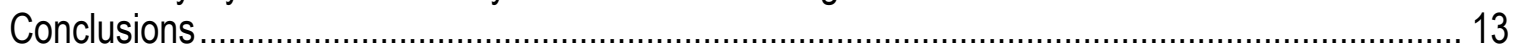

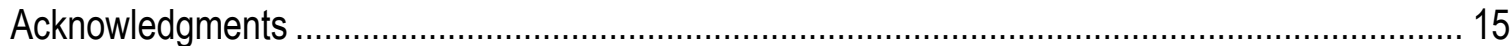

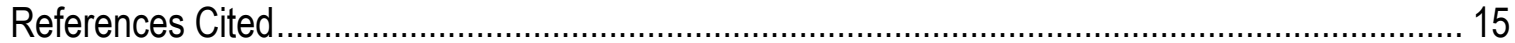

\section{Tables}

Table 1. Selected near-fault ground motion records.

Table 2. Natural periods of vibration obtained from the peak-picking (PP) method, the combined deterministic-stochastic subspace (DSS) method, and the computer model for the nine-story symmetric-plan building.

\section{Figures}

Figure 1. Distribution of magnitude $\left(M_{w}\right)$ and closest fault distance $\left(R_{r u p}\right)$ for the 30 near-fault ground-motion records selected.

Figure 2. A, Reference axes for the fault and the instrument with relevant angles noted. $B$, Reference axis for the building.

Figure 3. Response spectra of 30 near-fault ground motions and their geometric-mean used as design spectrum for single-story systems: fault parallel (strike parallel) components (left top panel), fault normal (strike normal) components (right top panel), comparison of median spectra of fault normal and fault parallel components (bottom panel). Damping ratio is 5 percent. $g$, acceleration due to gravity; sec, seconds.

Figure 4. Trace of deformation $(u)$ orbit of a two-degrees-of-freedom system (elastic vibration period, $T_{n}=1$ second) with direction-independent stiffness and damping subjected to the fault-normal/fault parallel (FN/FP) components of a ground motion.

Figure 5. For 30 near-fault ground-motion pairs listed in table 1, polar plots of spectral accelerations as a function of the rotation angle, $\theta_{\mathrm{x}}$, are shown for elastic single-degreeof-freedom (SDF) systems with vibration period $\left(T_{n}\right)$ equal to $0.2,1,2,3$, and 5 seconds (damping ratio 5 percent). The red lines represent the median spectral acceleration value $\left(A_{n}\right) \pm$ one standard deviation $\left(\sigma_{n}\right)$. The blue points correspond to pairs of maximum direction angle $\theta_{m}$ and spectral acceleration values $A_{m}$. The blue circles represent the median spectral acceleration value $\pm \sigma_{m}$ in the maximum direction. Note that except for short-period SDF systems ( $T_{n}=0.2$ seconds), $A_{n}$ values are generally polarized with faultnormal $\left(90^{\circ}\right)$ direction; however, $\theta_{m}$ shows large scattering with no correlation with fault- 
normal direction; also spectral acceleration values, $A_{m}$, corresponding to the maximum direction angle, $\theta_{m}$, are generally higher than median spectral acceleration value, $A_{n}$.

Figure 6. Polar plots of identified velocity pulse periods and spectral acceleration (acc.) values as a function of the rotation angle, $\theta_{x}$, for ground-motion (GM) pairs 1 to 15 (see table 1). The red dots show the directions in which velocity pulses are identified with their corresponding pulse periods. The filled gray area shows range of $\theta_{x}$ in which velocity pulses are observed. The dashed blue lines show spectral acceleration values for the maximum identified pulse period (damping ratio 5 percent). The blue line identifies the maximum direction. Numerical values for maximum pulse periods and maximum spectral accelerations are presented in the upper right corner of each subplot. Empty polar plot indicates that no velocity pulse is identified.

Figure 7. Polar plots of identified velocity pulse periods and spectral acceleration (acc) values as a function of the rotation angle, $\theta_{\mathrm{x}}$, for ground-motion (GM) pairs 16 to 30 (see table 1). The red dots show the directions in which velocity pulses are identified with their corresponding pulse periods. The filled gray area shows range of $\theta_{x}$ in which velocity pulses are observed. The dashed blue lines show spectral acceleration values for the maximum identified pulse period (damping ratio 5 percent). The blue line identifies the maximum direction. Numerical values for maximum pulse periods and maximum spectral accelerations are presented in the upper right corner of each subplot. Empty polar plot indicates that no velocity pulse is identified.

Figure 8. Schematic isometric and plan views of the selected single-story structural systems with three-degrees-of-freedom noted; buckling-restrained braced frames are highlighted..... 26

Figure 9. Constitutive model used for the buckling-restrained braces (BRBs).......................... 27

Figure 10. Periods and modes of vibration of the single-story three-degrees-of-freedom systems $\left(T_{n}=\right.$ elastic vibration period for the $n_{\text {th }}$ mode $)$.

Figure 11. Effective modal masses, $M_{n}{ }^{*}$, of the plan-A (symmetric-plan) and plan-B (asymmetric-plan) buildings. \%, percent.

Figure 12. Nine-story symmetric-plan building in Aliso Viejo, California. $A$, three-dimensional computer model. $B$, Photograph of the building looking to north elevation........................... 28

Figure 13. West elevation $(A)$ and typical floor plan $(B)$ of symmetric-plan building.................. 29

Figure 14. Locations of sensors shown with red arrows in symmetric-plan building. A, Plan view. $B$, south elevation.

Figure 15. Floor total accelerations recorded by the sensors shown in figure 14 during the 2008 magnitude 5.4 Chino Hills southern California earthquake......................................... 31

Figure 16. Comparison of normalized natural vibration modes identified by the deterministicstochastic subspace (DSS) method with modes of the computer model for symmetric-plan building ( ${ }^{*} \mathrm{WF}=$ west frame; ${ }^{* *} \mathrm{EW}=$ east frame).

Figure 17. First triplet of periods and modes of vibration of symmetric-plan building (only roof

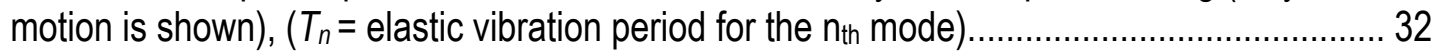

Figure 18. Effective modal masses, $M_{n}{ }^{*}$, for symmetric-plan building. \%, percent. ................... 33

Figure 19. Comparison of recorded and computed floor displacements at the sensor locations (fig. 14) of symmetric-plan building. Recorded data is from the 2008 magnitude 5.4 Chino Hills southern California earthquake.

Figure 20. Nine-story asymmetric-plan building. $A$, three-dimensional computer model. $B$, plan view. 
Figure 21. Trilinear force-deformation relations considering in-cycle strength deterioration (strength loss).

Figure 22. Krawinkler model for panel zones adapted from Computers and Structures, Inc. (2006).

Figure 23. First six natural periods and modes of vibration of asymmetric-plan building; $x$ lateral, $y$-lateral and torsional motions are shown in the three columns.

Figure 24. First triplet of periods and modes of vibration of asymmetric-plan building (only roof motion is shown), $\left(T_{n}=\right.$ elastic vibration period for the $n_{\text {th }}$ mode).

Figure 25. Effective modal masses, $M_{n}{ }^{*}$, for asymmetric-plan building. \%, percent 37

Figure 26. Displacement, $u_{x}$, at the center of mass (red line) as a function of the rotation angle, $\theta_{\mathrm{x}}$, for single-story symmetric-plan systems with elastic first-mode vibration period $\left(T_{n}\right)=2,3$, and 5 seconds subjected to ground motions (GM) with velocity pulse period close to $T_{n}$. The filled gray area shows values of $\theta_{x}$ in which velocity pulses are identified. Angles $\theta_{x}=0^{\circ}$ and $90^{\circ}$ correspond to the fault-parallel and fault-normal directions, respectively.

Figure 27. Floor total accelerations, $\ddot{u}_{x}^{t}$, at the center of mass (red line) as a function of the rotation angle, $\theta_{x}$, for single-story symmetric-plan systems with elastic first-mode vibration period $\left(T_{n}\right)=2,3$, and 5 seconds subjected to ground motions $(G M)$ with velocity pulse period close to $T_{n}$. The filled gray area shows values of $\theta_{x}$ in which velocity pulses are identified for each record. Angles $\theta_{x}=0^{\circ}$ and $90^{\circ}$ correspond to the fault-parallel and faultnormal directions, respectively.

Figure 28. Force in bracing $b 1$ (red line) as a function of the rotation angle, $\theta_{x}$, for singlestory symmetric-plan systems with elastic first-mode vibration period $\left(T_{n}\right)=2,3$, and 5 seconds subjected to ground motions (GM) with velocity pulse period close to $T_{n}$. The filled gray area shows values of $\theta_{x}$ in which velocity pulses are identified for each record. Angles $\theta_{x}=0^{\circ}$ and $90^{\circ}$ correspond to the fault-parallel and fault-normal directions, respectively.

Figure 29. Plastic deformation in bracing b1 (red line) as a function of the rotation angle, $\theta_{x}$, for single-story symmetric-plan systems with elastic first-mode vibration period $\left(T_{n}\right)=2,3$, and 5 seconds subjected to ground motions (GM) with velocity pulse period close to $T_{n}$. The filled gray area shows values of $\theta_{x}$ in which velocity pulses are identified for each record. Angles $\theta_{x}=0^{\circ}$ and $90^{\circ}$ correspond to the fault-parallel and fault-normal directions, respectively.

Figure 30. Displacement, $u_{x}$, at the center of mass (red line) as a function of the rotation angle, $\theta_{x}$, for single-story asymmetric-plan systems with elastic first-mode vibration period $\left(T_{n}\right)=2,3$, and 5 seconds subjected to ground motions $(G M)$ with velocity pulse period close to $T_{n}$. The filled gray area shows values of $\theta_{x}$ in which velocity pulses are identified for each record. Angles $\theta_{x}=0^{\circ}$ and $90^{\circ}$ correspond to the fault-parallel and fault-normal directions, respectively.

Figure 31. Displacement, $u_{x}$, at corner p2 (red line) as a function of the rotation angle, $\theta_{x}$, for single-story asymmetric-plan systems with elastic first-mode vibration period $\left(T_{n}\right)=2,3$, and 5 seconds subjected to ground motions (GM) with velocity pulse period close to $T_{n}$. The filled gray area shows values of $\theta_{x}$ in which velocity pulses are identified for each 
record. Angles $\theta_{x}=0^{\circ}$ and $90^{\circ}$ correspond to the fault-parallel and fault-normal directions, respectively.

Figure 32. Floor total accelerations, $\ddot{u}_{x}^{t}$, at corner $\mathrm{p} 2$ (red line) as a function of the rotation angle, $\theta_{x}$, for asymmetric-plan buildings with elastic first-mode vibration period $\left(T_{n}\right)=2,3$, and 5 seconds subjected to ground motions (GM) with velocity pulse period close to $T_{n}$. The filled gray area shows values of $\theta_{x}$ in which velocity pulses are identified for each record. Angles $\theta_{x}=0^{\circ}$ and $90^{\circ}$ correspond to the fault-parallel and fault-normal directions, respectively.

Figure 33. Force in bracing $b 3$ (red line) as a function of the rotation angle, $\theta_{x}$, for singlestory asymmetric-plan systems with elastic first-mode vibration period $\left(T_{n}\right)=2,3$, and 5 seconds subjected to ground motions (GM) with velocity pulse period close to $T_{n}$. The filled gray area shows values of $\theta_{x}$ in which velocity pulses are identified for each record. Angles $\theta_{x}=0^{\circ}$ and $90^{\circ}$ correspond to the fault-parallel and fault-normal directions, respectively.

Figure 34. Plastic deformation in bracing b3 (red line) as a function of the rotation angle, $\theta_{x}$, for single-story asymmetric-plan systems with elastic first-mode vibration period $\left(T_{n}\right)=2$, 3 , and 5 seconds subjected to ground motions (GM) with velocity pulse period close to $T_{n}$. The filled gray area shows values of $\theta_{x}$ in which velocity pulses are identified for each record. Angles $\theta_{x}=0^{\circ}$ and $90^{\circ}$ correspond to the fault-parallel and fault-normal directions, respectively.

Figure 35. Median displacements, $u_{x}$, at the center of mass as a function of the rotation angle, $\theta_{x}$, for single-story symmetric-plan systems with elastic first-mode vibration period $\left(T_{n}\right)=0.2,1,2,3$, and 5 seconds subjected to bidirectional loading. The red lines represent the median displacement $u_{x} \pm \sigma$. The blue circles represent the median displacement, $u_{x m} \pm \sigma$, for the systems subjected to bidirectional ground motions in the maximum direction. Solid lines are 50th and dashed lines are16th and 84th percentile engineering demand parameters (EDPs). MD, maximum direction.

Figure 36. Median total-accelerations, $\ddot{u}_{x}{ }$, at the center of mass as a function of the rotation angle, $\theta_{\mathrm{x}}$, for single-story symmetric-plan systems with elastic first-mode vibration period $\left(T_{n}\right)=0.2,1,2,3$, and 5 seconds subjected to bidirectional loading. The red lines represent the median displacement, $\ddot{u}_{x} \pm \sigma$. The blue circles represent the median totalacceleration, $\ddot{u}_{x m} \pm \sigma$, for the systems subjected to bidirectional ground motions in the maximum direction. Solid lines are 50th and dashed lines are16th and 84th percentile engineering demand parameters (EDPs). MD, maximum direction.

Figure 37. Median force $q_{1}$ in bracing b1 as a function of the rotation angle, $\theta_{x}$, for singlestory symmetric-plan systems elastic first-mode vibration period $\left(T_{n}\right)=0.2,1,2,3$, and 5 seconds subjected to bidirectional loading. The red lines represent the median force, $q_{1} \pm$ $\sigma$. The blue circles represent the median force, $q_{1} \pm \sigma$, for the systems subjected to bidirectional ground motions in the maximum direction. Solid lines are 50th and dashed lines are16th and 84th percentile engineering demand parameters (EDPs). MD, maximum direction

Figure 38. Median displacements, $u_{x}$, at the center of mass as a function of the rotation angle, $\theta_{x}$, for single-story asymmetric-plan systems with elastic first-mode vibration period $\left(T_{n}\right)=0.2,1,2,3$, and 5 seconds subjected to bidirectional loading. The red lines 
represent the median displacement $u_{x} \pm \sigma$. The blue circles represent the median displacement $u_{x m} \pm \sigma$ for the systems subjected to bidirectional ground motions in the maximum direction. Solid lines are 50th and dashed lines are16th and 84th percentile engineering demand parameters (EDPs). MD, maximum direction.

Figure 39. Median displacements, $u_{x}$, at corner $\mathrm{p} 2$ as a function of the rotation angle, $\theta_{x}$, for single-story asymmetric-plan systems with elastic first-mode vibration period $\left(T_{n}\right)=0.2,1$, 2,3 , and 5 seconds subjected to bidirectional loading. The red lines represent the median displacement, $u_{x} \pm \sigma$. The blue circles represent the median displacement, $u_{x m} \pm \sigma$, for the systems subjected to bidirectional ground motions in the maximum direction. Solid lines are 50th and dashed lines are16th and 84th percentile engineering demand parameters (EDPs). MD, maximum direction.

Figure 40. Median total-accelerations, $\ddot{u}_{x}^{t}$, at corner $\mathrm{p} 2$ as a function of the rotation angle, $\theta_{\mathrm{x}}$, for single-story asymmetric-plan systems with elastic first-mode vibration period $\left(T_{n}\right)=$ $0.2,1,2,3$, and 5 seconds subjected to bidirectional loading. The red lines represent the median displacement, $\ddot{u}_{x} \pm \sigma$. The blue circles represent the median total-acceleration, $\ddot{u} t_{x m} \pm \sigma$, for the systems subjected to bidirectional ground motions in the maximum direction. Solid lines are 50th and dashed lines are16th and 84th percentile engineering demand parameters (EDPs). MD, maximum direction.

Figure 41. Median force, $q_{1}$, in bracing b3 as a function of the rotation angle, $\theta_{x}$, for singlestory asymmetric-plan systems with elastic first-mode vibration period $\left(T_{n}\right)=0.2,1,2,3$, and 5 seconds subjected to bidirectional loading. The red lines represent the median force, $q_{1} \pm \sigma$. The blue circles represent the median force, $q_{1} \pm \sigma$, for the systems subjected to bidirectional ground motions in the maximum direction. Solid lines are 50th and dashed lines are16th and 84th percentile engineering demand parameters (EDPs). MD, maximum direction.

Figure 42. Story drifts, floor total accelerations, and internal forces as a function of the rotation angle, $\theta_{x}$, for the linear nine-story symmetric-plan building (first-mode elastic vibration period, $T_{1}=1.51$ seconds) subjected to ground-motion (GM) no. 9, which has maximum velocity pulse period of 1.9 seconds. The filled gray area shows values of $\theta_{x}$ in which velocity pulses are identified. Angles $\theta_{x}=0^{\circ}$ and $90^{\circ}$ correspond to the fault-parallel and fault-normal directions, respectively.

Figure 43. Height-wise distribution of median and dispersion values of story drift in the $x$ and $y$ directions for the linear nine-story symmetric-plan building. The gray, red, and blue lines show median and dispersion values of story drift due to bidirectional ground motions in arbitrary orientations in the fault-parallel and fault-normal directions (FN-FP) and in the maximum direction (MD), respectively. \%, percent; disp., displacement.

Figure 44. Height-wise distribution of median and dispersion values of floor total acceleration in the $x$ and $y$ directions for the linear nine-story symmetric-plan building. The gray, red, and blue lines show median and dispersion values of floor total accelerations due to bidirectional ground motions in arbitrary orientations in the fault-parallel and faultnormal directions (FN-FP) and in the maximum direction (MD), respectively. \%, percent; disp., displacement.

Figure 45. Height-wise distribution of median and dispersion values of moments at the beams highlighted in figure 13 for the linear nine-story symmetric-plan building. The gray, red, and blue lines show median and dispersion values of beam moments due to 
bidirectional ground motions in arbitrary orientations in the fault-parallel and fault-normal directions (FN-FP) and in the maximum direction (MD), respectively. \%, percent; disp., displacement.

Figure 46. Height-wise distribution of median and dispersion values of moments at the columns highlighted in figure 13 for the linear nine-story symmetric-plan building. The gray, red, and blue lines show median and dispersion values of beam moments due to bidirectional ground motions in arbitrary orientations in the fault-parallel and fault-normal directions (FN-FP) and in the maximum direction (MD), respectively. \%, percent; disp., displacement.

Figure 47. Median values of story drifts, floor total accelerations, and internal forces in the $x$ direction as a function of the rotation angle, $\theta_{x}$, for the linear nine-story symmetric-plan building subjected to bidirectional loading. The red lines represent the median engineering demand parameter (EDP) values $\pm \sigma$. The blue circles represent the median EDP values $\pm \sigma$ for the building subjected to bidirectional ground motions in the maximum direction. Solid lines are 50th and dashed lines are16th and 84th percentile EDPs.

Figure 48. Median values of story drifts, floor total accelerations, and internal forces in the $y$ direction as a function of the rotation angle, $\theta_{x}$, for the linear nine-story symmetric-plan building subjected to bidirectional loading. The red lines represent the median engineering demand parameter (EDP) values $\pm \sigma$. The blue circles represent the median EDP values $\pm \sigma$ for the building subjected to bidirectional ground motions in the maximum direction. Solid lines are 50th and dashed lines are16th and 84th percentile EDPs.

Figure 49. Story drifts, floor total accelerations, and internal forces as a function of the rotation angle, $\theta_{x}$, for the linear nine-story asymmetric-plan building (elastic first-mode vibration period, $T_{1}=2.5$ seconds) subjected to ground motion (GM) 2 , which has maximum velocity pulse period of 2.4 seconds. The filled gray area shows values of $\theta_{x}$ in which velocity pulses are identified. Angles $\theta_{\mathrm{x}}=0^{\circ}$ and $90^{\circ}$ correspond to the fault-parallel and fault-normal directions, respectively.

Figure 50. Height-wise distribution of median and dispersion values of story drift at the corner highlighted in figure 20 in the $x$ and $y$ directions for the linear nine-story asymmetric-plan building. The gray, red, and blue lines show median and dispersion values of story drift due to bidirectional ground motions in arbitrary orientations in the fault-parallel and fault-normal directions (FN-FP) and in the maximum direction (MD), respectively. \%, percent; disp., displacement.

Figure 51. Height-wise distribution of median and dispersion values of floor total acceleration in the $x$ and $y$ directions for the linear nine-story asymmetric-plan building. The gray, red, and blue lines show median and dispersion values of floor total accelerations due to bidirectional ground motions in arbitrary orientations in the faultparallel and fault-normal directions (FN-FP) and in the maximum direction (MD), respectively. \%, percent; disp., displacement.

Figure 52. Height-wise distribution of median and dispersion values of moments at the beams highlighted in figure 20 for the linear nine-story asymmetric-plan building. The gray, red, and blue lines show median and dispersion values of beam moments due to bidirectional ground motions in arbitrary orientations in the fault-parallel and fault-normal directions (FN-FP) and in the maximum direction (MD), respectively. \%, percent; disp., displacement. 
Figure 53. Height-wise distribution of median and dispersion values of moments at the columns highlighted in figure 20 for the linear nine-story asymmetric-plan building. The gray, red, and blue lines show median and dispersion values of beam moments due to bidirectional ground motions in arbitrary orientations in the fault-parallel and fault-normal directions (FN-FP) and in the maximum direction (MD), respectively. \%, percent; disp., displacement.

Figure 54. Median values of story drifts at the corner, floor total accelerations, and internal forces in the $x$ direction as a function of the rotation angle, $\theta_{x}$, for the linear nine-story asymmetric-plan building subjected to bidirectional loading. The red lines represent the median engineering demand parameter (EDP) values $\pm \sigma$. The blue circles represent the median EDP values $\pm \sigma$ for the building subjected to bidirectional ground motions in the maximum direction. Solid lines are Solid lines are $50^{\text {th }}$ and dashed lines are $16^{\text {th }}$ and $84^{\text {th }}$ percentile EDPs.

Figure 55. Median values of story drifts, floor total accelerations, and internal forces in the $y$ direction as a function of the rotation angle, $\theta_{x}$, for the linear nine-story asymmetric-plan building subjected to bidirectional loading. The red lines represent the median engineering demand parameter (EDP) values $\pm \sigma$. The blue circles represent the median EDP values $\pm \sigma$ for the building subjected to bidirectional ground motions in the maximum-direction. Solid lines are $50^{\text {th }}$ and dashed lines are $16^{\text {th }}$ and $84^{\text {th }}$ percentile EDPs.

Figure 56. Story drifts, total chord rotations, and internal forces as a function of the rotation angle, $\theta_{x}$, for the nonlinear nine-story symmetric-plan building (first-mode elastic vibration period, $T_{1}=1.51$ seconds) subjected to ground motion (GM) 9 , which has maximum velocity pulse period of 1.9 seconds. The filled gray area shows values of $\theta_{\mathrm{x}}$ in which velocity pulses are identified. Angles $\theta_{\mathrm{x}}=0^{\circ}$ and $90^{\circ}$ correspond to the fault-parallel and fault-normal directions, respectively.

Figure 57. Height-wise distribution of median $+\sigma$ and dispersion values of story drift in the $x$ and $y$ directions for the nonlinear nine-story symmetric-plan building. The gray, red, and blue lines show median $+\sigma$ and dispersion values of story drift due to bidirectional ground motions in arbitrary orientations in the fault-parallel and fault-normal directions (FN-FP) and in the maximum direction (MD), respectively. \%, percent; disp., displacement.

Figure 58. Height-wise distribution of median $+\sigma$ and dispersion values of total chord rotation in the $x$ and $y$ directions for the nonlinear nine-story symmetric-plan building. The gray, red, and blue lines show median $+\sigma$ and dispersion values of floor total accelerations due to bidirectional ground motions in arbitrary orientations in the fault-parallel and faultnormal directions (FN-FP) and in the maximum direction (MD), respectively. \%, percent; disp., displacement.

Figure 59. Height-wise distribution of median $+\sigma$ and dispersion values of moments at the beams highlighted in figure 13 for the nonlinear nine-story symmetric-plan building. The gray, red, and blue lines show median $+\sigma$ and dispersion values of beam moments due to bidirectional ground motions in arbitrary orientations in the fault-parallel and fault-normal directions (FN-FP) and in the maximum direction (MD), respectively. \%, percent; disp., displacement. 
Figure 60. Height-wise distribution of median $+\sigma$ and dispersion values of moments at the columns highlighted in figure 13 for the nonlinear nine-story symmetric-plan building. The gray, red, and blue lines show median $+\sigma$ and dispersion values of beam moments due to bidirectional ground motions in arbitrary orientations in the fault-parallel and fault-normal directions (FN-FP) and in the maximum direction (MD), respectively. \%, percent; disp., displacement.

Figure 61. Median values of story drifts, total chord rotations, and internal forces in the $x$ direction as a function of the rotation angle, $\theta_{x}$, for the nonlinear nine-story symmetricplan building subjected to bidirectional loading. The red lines represent the median engineering demand parameter (EDP) values $\pm \sigma$. The blue circles represent the median EDP values $\pm \sigma$ for the building subjected to bidirectional ground motions in the maximum direction. Solid lines are $50^{\text {th }}$ and dashed lines are $16^{\text {th }}$ and $84^{\text {th }}$ percentile EDPs.

Figure 62. Median values of story drifts, total chord rotations, and internal forces in the $y$ direction as a function of the rotation angle, $\theta_{\mathrm{x}}$, for the nonlinear nine-story symmetricplan building subjected to bidirectional loading. The red lines represent the median engineering demand parameter (EDP) values $\pm \sigma$. The blue circles represent the median EDP values $\pm \sigma$ for the building subjected to bidirectional ground motions in the maximum direction. Solid lines are $50^{\text {th }}$ and dashed lines are $16^{\text {th }}$ and $84^{\text {th }}$ percentile EDPs.

Figure 63. Story drifts, total chord rotations, and internal forces as a function of the rotation angle, $\theta_{\mathrm{x}}$, for the nonlinear nine-story asymmetric-plan building $\left(T_{1}=2.5 \mathrm{~s}\right)$ subjected to ground motion 2, which has maximum velocity pulse period of 2.4 seconds. The filled gray area shows values of $\theta_{\mathrm{x}}$ in which velocity pulses are identified. Angles $\theta_{\mathrm{x}}=0^{\circ}$ and $90^{\circ}$ correspond to the fault-parallel and fault-normal directions, respectively.

Figure 64. Height-wise distribution of median $+\sigma$ and dispersion values of story drift at the corner highlighted in figure 20 in the $x$ and $y$ directions for the nonlinear nine-story asymmetric-plan building. The gray, red, and blue lines show median $+\sigma$ and dispersion values of story drift due to bidirectional ground motions in arbitrary orientations in the fault-parallel and fault-normal directions (FN-FP) and in the maximum direction (MD), respectively. \%, percent; disp., displacement.

Figure 65. Height-wise distribution of median $+\sigma$ and dispersion values of total chord rotation in the $x$ and $y$ directions for the nonlinear nine-story asymmetric-plan building. The gray, red, and blue lines show median $+\sigma$ and dispersion values of floor total accelerations due to bidirectional ground motions in arbitrary orientations in the fault-parallel and faultnormal directions (FN-FP) and in the maximum direction (MD), respectively. \%, percent; disp., displacement.

Figure 66. Height-wise distribution of median+ $\sigma$ and dispersion values of moments at the beams highlighted in figure 20 for the nonlinear nine-story asymmetric-plan building. The gray, red, and blue lines show median $+\sigma$ and dispersion values of beam moments due to bidirectional ground motions in arbitrary orientations in the fault-parallel and fault-normal directions (FN-FP) and in the maximum direction (MD), respectively. \%, percent; disp., displacement.

Figure 67. Height-wise distribution of median $+\sigma$ and dispersion values of moments at the columns highlighted in figure 20 for the nonlinear nine-story asymmetric-plan building. The gray, red, and blue lines show median $+\sigma$ and dispersion values of beam moments 
due to bidirectional ground motions in arbitrary orientations in the fault-parallel and faultnormal directions (FN-FP) and in the maximum direction (MD), respectively. \%, percent; disp., displacement.

Figure 68. Median values of story drifts at the corner, total chord rotations, and internal forces in the $x$ direction as a function of the rotation angle, $\theta_{x}$, for the nonlinear nine-story asymmetric-plan building subjected to bidirectional loading. The red lines represent the median engineering demand parameter (EDP) values $\pm \sigma$. The blue circles represent the median EDP values $\pm \sigma$ for the building subjected to bidirectional ground motions in the maximum direction. Solid lines are $50^{\text {th }}$ and dashed lines are $16^{\text {th }}$ and $84^{\text {th }}$ percentile EDPs.

Figure 69. Median values of story drifts, total chord rotations, and internal forces in the $y$ direction as a function of the rotation angle, $\theta_{x}$, for the nonlinear nine-story asymmetricplan building subjected to bidirectional loading. The red lines represent the median-EDP values $\pm \sigma$. The blue circles represent the median-EDP values $\pm \sigma$ for the building subjected to bidirectional ground motions in the maximum direction. Solid lines are $50^{\text {th }}$ and dashed lines are $16^{\text {th }}$ and $84^{\text {th }}$ percentile EDPs. 
This page intentionally left blank 


\title{
Should Ground-Motion Records be Rotated to Fault- Normal/Parallel or Maximum Direction for Response History Analysis of Buildings?
}

\author{
By Juan C. Reyes ${ }^{1}$ and Erol Kalkan ${ }^{2}$
}

\begin{abstract}
In the United States, regulatory seismic codes (for example, California Building Code) require at least two sets of horizontal ground-motion components for three-dimensional (3D) response history analysis (RHA) of building structures. For sites within 5 kilometers (3.1 miles) of an active fault, these records should be rotated to fault-normal and fault-parallel (FN/FP) directions, and two RHAs should be performed separately — when FN and then FP direction are aligned with transverse direction of the building axes. This approach is assumed to lead to two sets of responses that envelope the range of possible responses over all nonredundant rotation angles. The validity of this assumption is examined here using 3D computer models of singlestory structures having symmetric (torsionally stiff) and asymmetric (torsionally flexible) layouts subjected to an ensemble of near-fault ground motions with and without apparent velocity pulses. In this parametric study, the elastic vibration period is varied from 0.2 to 5 seconds, and yieldstrength reduction factors, $R$, are varied from a value that leads to linear-elastic design to 3 and 5 . Further validations are performed using 3D computer models of 9-story structures having symmetric and asymmetric layouts subjected to the same ground-motion set. The influence of the ground-motion rotation angle on several engineering demand parameters (EDPs) is examined in both linear-elastic and nonlinear-inelastic domains to form benchmarks for evaluating the use of the FN/FP directions and also the maximum direction (MD). The MD ground motion is a new definition for horizontal ground motions for use in site-specific ground-motion procedures for seismic design according to provisions of the American Society of Civil Engineers/Seismic Engineering Institute (ASCE/SEI) 7-10. The results of this study have important implications for current practice, suggesting that ground motions rotated to MD or FN/FP directions do not necessarily provide the most critical EDPs in nonlinear-inelastic domain; however, they tend to produce larger EDPs than as-recorded (arbitrarily oriented) motions.
\end{abstract}

\section{Introduction}

In the United States, both the International Building Code (ICBO, 2009) and the California Building Code (CBC2010) (ICBO, 2010) refer to American Society of Civil Engineers/Seismic Engineering Institute (ASCE/SEI) 7-05 chapter 16 (ASCE, 2006) when response history analysis (RHA) is required for design verification of building structures. These

\footnotetext{
${ }^{1}$ Universidad de los Andes, Bogota, Colombia.

${ }^{2}$ U.S. Geological Survey, Menlo Park, California.
} 
guidelines require at least two sets of horizontal ground-motion components for threedimensional (3D) RHA of structures. According to section 1615A.1.25 of the California Building Code, at sites within 5 kilometers (km) (3.1 miles, mi) from an active fault that dominates the earthquake hazard, each pair of ground-motion components shall be rotated to the fault-normal and fault-parallel (FN/FP) directions (also called the strike-normal and strikeparallel directions) for 3D RHAs. It is believed that the angle corresponding to the FN/FP directions will lead to the most critical structural response. This assumption is based on the fact that, in the proximity of an active fault system, ground motions are significantly affected by the faulting mechanism, direction of rupture propagation relative to the site, as well as the possible static deformation of the ground surface associated with fling-step effects (Bray and RodriguezMarek, 2004; Kalkan and Kunnath, 2006), and these near-source effects cause most of the seismic energy from the rupture to arrive in a single coherent long-period pulse of motion in the FN/FP directions (Mavroeidis and Papageorgiou, 2003; Kalkan and Kunnath, 2007, 2008). Thus, rotating ground-motion pairs to FN/FP directions is assumed to be a conservative approach, appropriate for design verification of new structures.

The provision for rotating ground-motion records to FN/FP directions in the CBC2010 is absent in the ASCE/SEI 7-05 guidelines. However, this modification is now included in the most recent ASCE/SEI 7-10 (ASCE, 2010) standards, which have additional proposed changes to be incorporated in the new generation of the building codes. Two of the changes are the use of maximum direction (MD) ground motion and a revised definition of horizontal ground motions used in site-specific ground-motion procedures for seismic design (chapter 21 of ASCE/SEI 710). The MD is the direction of the rotated ground-motion pair that results in maximum linearelastic response quantity of a single lumped mass oscillator (free to vibrate in both horizontal directions) over exhaustive record rotation. The assumptions behind the MD ground motions are that the structural properties including stiffness and strength are identical in all directions, and the azimuth of the MD ground motion coincides with the structure's principal axes (Singh and others, 2011). Although the first assumption may be true for purely symmetric-plan structures (such as silos, oil tanks, communication poles, elevated water tanks, and guyed towers), it may not be valid for other systems whose response is dominated by modes of vibration along specific axes. The second assumption on the other hand refers to ground motions with a lower probability of occurrence-it is very unlikely that ground-motion incidence angle (angle of attack) with respect to the building's transverse direction is same as its MD. In chapter 21 of the ASCE/SEI 7-10, the concept of MD is used to develop a MD response spectrum to be used for seismic design. In the MD spectrum, spectral ordinates at each period can be in a different orientation because the maximum motion varies with the period of the oscillator. Because of these issues, use of MD ground motions for seismic design is found to be controversial, and it is argued that it would result in 10 to 30 percent overestimation of design ground-motion level (Stewart and others, 2011).

The idea of rotating ground-motion pairs to certain axes, critical for response, is not new; it has been studied previously in various contexts. Penzien and Watabe (1975) defined the principal axes of a pair of ground motions as the angle or axis along which the two horizontal components are uncorrelated. The principal axis is independent of the vibration period (Goda, 2012) and is not correlated with the MD (Hong and Goda, 2010). Using this idea of principal axis, the effects of seismic rotation angle, defined as the angle between the principal axes of the ground-motion pair and the structural axes along which structural response was studied (Fernandez-Davilla and others, 2000; MacRae and Matteis, 2000; Tezcan and Alhan, 2001; 
Khoshnoudian and Poursha, 2004; Rigato and Medina, 2007; Lagaros, 2010; Goda, 2012), previous studies demonstrate that the rotation angle of ground motions influences the structural response significantly and that the angle that yields the peak response over all possible nonredundant angles, called $\theta_{\text {critical }}$ (or $\theta_{c r}$ ) depends on the seismic excitation level and character of shaking. A formula for deriving $\theta_{c r}$ was proposed by Wilson (1995). Other researchers have improved on the closed-form solution of Wilson (1995) by accounting for the statistical correlation of horizontal components of ground motion in an explicit way (Lopez and Tores, 1997; Lopez and others, 2000). However, the Wilson (1995) formula is based on concepts from response spectrum analysis - an approximate procedure used to estimate structural responses in the linear-elastic domain. Focusing on linear-elastic multi-degree-of-freedom symmetric- and asymmetric-plan structures, Athanatopoulou (2004) investigated the effect of the rotation angle on structural response using linear RHAs and provided formulas for determining the maximum response over all rotation angles given the linear-elastic response histories for two orthogonal orientations. The analysis results have shown that, for the records used, the critical value of an EDP can be as much as 80 percent larger than the usual response produced when the groundmotion components are applied along the structural axes. Athanatopoulou (2004) also concluded that the critical angle corresponding to peak response over all angles varies not only with the ground-motion pair under consideration but also with the response quantity of interest. These findings are confirmed in Kalkan and Kwong (2012 and in press) where the impacts of groundmotion rotation angle including FN/FP directions on several different EDPs have been examined using a linear-elastic 3D computer model of a multistory building.

Previous studies investigated response behavior of either linear-elastic multi-degree-offreedom buildings or nonlinear-inelastic single-degree-of-freedom systems. Because there is still a lack of research addressing bidirectional nonlinear response of multi-degrees-of-freedom systems considering ground-motion directionality effects, this study systematically evaluates whether ground motions rotated to MD or FN/FP directions lead to the most critical estimates of EDPs from RHAs. For this purpose, 3D computer models of single and multistory structures having symmetric (torsionally stiff) and asymmetric (torsionally flexible) layouts are subjected to an ensemble of bidirectional near-fault ground motions with and without apparent velocity pulses. Also investigated are the rotation angle of an apparent velocity pulse and its correlation with the MD and FN/FP directions. At the end, this study provides recommendations regarding the use of MD and FN/FP directions to rotate ground-motion records for RHA of building structures.

\section{Ground Motions Selected}

For this investigation, 30 near-fault strong-motion pairs of records, listed in table 1, were selected from nine shallow crustal earthquakes (see fig. 1) compatible with the following scenario:

- $\quad$ Moment magnitude: $M_{\mathrm{w}}=6.7 \pm 0.2$

- Closest-fault distance ${ }^{3}: 0.1 \leq R_{\text {rup }} \leq 15 \mathrm{~km}^{4}$

- Record highest usable period ${ }^{5} \geq 6$ seconds

\footnotetext{
${ }^{3} R_{\text {rup }}=$ closest fault distance from a site to a coseismic rupture plane.

${ }^{4}$ Although code requires ground motions to be rotated for sites within $5 \mathrm{~km}$ of fault only, due to lack of available records within this distance range, we selected records from as far as15 km.
} 
These ground motions were rotated to fault-normal (FN) and fault-parallel (FP) orientations using the following transformation equations:

$$
\begin{aligned}
& \ddot{u}_{F P}=\ddot{u}_{1} \cos \left(\beta_{1}\right)+\ddot{u}_{2} \cos \left(\beta_{2}\right) \\
& \ddot{u}_{F N}=\ddot{u}_{1} \sin \left(\beta_{1}\right)+\ddot{u}_{2} \sin \left(\beta_{2}\right),
\end{aligned}
$$

where $\beta_{1}=\alpha_{\text {strike }}-\alpha_{1}, \beta_{2}=\alpha_{\text {strike }}-\alpha_{2}, \alpha_{\text {strike }}$ is the strike of the fault, $\alpha_{1}$ and $\alpha_{2}$ are the azimuths of the instrument axes as shown in figure $2 A$. Shown in figure 3 are the 5-percent-damped geometric-mean (termed "median" here after) response spectra for the FN and FP components of the ground motions. Figure 3 also shows the response spectra of the records. As expected, the ordinates of median spectra of FN components are larger than those of FP components, because the FN components of near-fault ground motions are generally stronger. The median spectrum of $30 \mathrm{FN}$ records is taken as the target spectrum for design of single-story symmetric and asymmetric structures to be used in a parametric study.

The ground motions (acceleration time series) were additionally rotated $\theta_{x}$ away from the $\mathrm{FP}$ axis as shown in figure $2 \mathrm{~B}$. The angle $\theta_{x}$ varies from $5^{\circ}$ to $360^{\circ}$ every $5^{\circ}$ in the clockwise direction. These rotations were conducted using equations (1) and (2) with the following modifications: (1) $\alpha_{1}$ and $\alpha_{2}$ were changed by $\theta_{x}$ and $\theta_{y}$, respectively, and (2) $\beta_{1}$ and $\beta_{2}$ were redefined as $\beta_{1}=\alpha_{\text {strike }}-\alpha_{1}-\theta_{x}$ and $\beta_{2}=\alpha_{\text {strike }}-\alpha_{2}-\theta_{y}$. The $\mathrm{x}$ - and $\mathrm{y}$-axis as well as the angles $\theta_{x}$ and $\theta_{y}$ are shown in figure $2 B$.

Figure 4 shows the response of a two-degrees-of-freedom system with equal stiffness and damping ratio in the $x$ and $y$ axes subjected to the FN/FP components of a ground motion (that is, $\theta_{x}=0$ ). The maximum deformation of this system occurs at an angle $\theta_{m}$ away from the FP axis. This new orientation for the response quantity of interest will be called in this research maximum direction (MD).

In the near-field of an active fault, seismic waves from the earthquake source are affected by forward-directivity and fling-step effects. These near-source effects cause most of the seismic energy from the rupture to arrive in a single coherent long-period velocity pulse. Ground motions having such a distinct pulse-like character arise in general at the beginning of the seismogram, and their effects tend to increase the spectral acceleration in the long-period part of the spectrum (Golesorkhi and Gouchon, 2002). Baker (2007) developed a numerical procedure to identify and characterize velocity pulses in ground-motion time series. We use his procedure to identify velocity pulses in rotated motions whose rotation angle is varied from $5^{\circ}$ to $360^{\circ}$ at an interval of $5^{\circ}$ in the clockwise direction. Results of this analysis are shown in figures 5 thru 7 . For 30 nearfault ground-motion pairs, figure 5 shows the polar plots of spectral acceleration values as a function of the rotation angle $\left(\theta_{\mathrm{x}}\right)$ for elastic single-degree-of-freedom (SDF) systems with vibration period $\left(T_{n}\right)$ equal to $0.2,1,2,3$, and 5 seconds (s). In this figure, the red lines represent the median spectral acceleration value $\pm \sigma_{\mathrm{n}}$ (that is, 16th, 50th, and 84th percentile), and the blue points correspond to pairs of maximum direction angle $\left(\theta_{m}\right)$ and spectral acceleration values $\left(A_{m}\right)$. The blue circles represent the median spectral acceleration value $\pm \sigma_{\mathrm{m}}$ in the MD. Except

\footnotetext{
${ }^{5}$ Low-cut corner frequency of the Butterworth filter applied; because the highest usable period is greater than 6 seconds, records in table 1 have enough long-period content to compute their spectra reliably up to 5 seconds.
} 
for short-period system ( $T_{n}=0.2$ seconds), median spectral acceleration values $\left(A_{n}\right)$ (red lines) are polarized with the fault-normal $\left(\theta_{x}=90^{\circ}\right)$ direction. Spectral acceleration values $\left(A_{m}\right)$, corresponding to the maximum direction angle $\left(\theta_{m}\right.$, ) are generally higher than median spectral acceleration value $\left(A_{n}\right)$, although they get closer as the period $\left(T_{n}\right)$ increases.

Studies of ground-motion directionality have shown that the azimuth of the MD ground motion is arbitrary for fault distances $\left(R_{\text {rup }}\right)$ larger than approximately 3-5 km (Campbell and Bozorgnia, 2007, Watson-Lamprey and Boore, 2007). At closer fault distances $\left(R_{r u p}<3-5 \mathrm{~km}\right)$, however, the azimuth of the maximum direction motion tends to align with the strike-normal direction (Watson-Lamprey and Boore, 2007; Huang and others, 2009). In contrast, $\theta_{m}$ in figure 5 shows large scattering with no visible correlation with the FN direction for records taken within $5 \mathrm{~km}$ of the fault. This finding indicates that the azimuth of the maximum direction motion does not necessarily align with the strike-normal direction even at closer fault distances $\left(R_{\text {rup }}<3-5 \mathrm{~km}\right)$.

The next set of figures (figs. 6 and 7) show the polar plot of identified velocity pulse periods and spectral accelerations as a function of the rotation angle, $\theta_{\mathrm{x}}$, for 30 ground-motion pairs in two groups (first 15 records are shown in fig. 6, and the remaining 15 records are shown in fig. 7). In these plots the red dots indicate (1) pulse periods scaled in polar coordinates and (2) directions in which the velocity pulses are identified. The filled gray area shows the range of $\theta_{x}$ with velocity pulses. The dashed blue lines show spectral acceleration values computed for SDF system with $T_{n}$ equal to the maximum pulse period of the ground-motion (GM) pair at 5 percent damping ratio (for example, for GM1 dashed blue lines correspond to spectral accelerations computed for SDF system with $T_{n}=4.9$ seconds under bidirectional excitation). The light blue line identifies the maximum direction angle, $\theta_{m}$. The numerical values for maximum pulse periods and maximum spectral accelerations are presented in the upper right corner of each subplot. An empty polar plot indicates that no velocity pulse is identified for a given groundmotion pair.

These two figures present important findings. First, let us consider the GM1 pair (fig. 6). This polar plot indicates that rotated motions have a maximum pulse period of 4.9 seconds. The apparent pulse is identified for $\theta_{x}$ in between $40^{\circ}-80^{\circ}$ and $130^{\circ}-170^{\circ}$, and it disappears at other angles including $90^{\circ}$ (fault-normal direction). For this record, maximum direction angle, $\theta_{m}$, is at $45^{\circ}$ and $135^{\circ}$, and the velocity pulse is also identified. Lastly, the maximum spectral acceleration of $0.2 \mathrm{~g}$ ( $\mathrm{g}=$ gravity) is observed at $\theta_{\mathrm{m}}$. In the FN direction, maximum spectral acceleration is decreased by 30 percent. Examinations of polar plots of all records permit the following observations:

1. Among 30 near-fault ground-motion records, velocity pulses are identified only for 75 percent of the records. For records with identified velocity pulses, one third of them have no pulses in the FN direction, indicating that FN direction does not always have an apparent velocity pulse.

2. For almost all GM pairs (except for GM 29), maximum direction angle, $\theta_{m}$, is in the direction where the velocity pulse is identified. This strong correlation shows that the maximum spectral acceleration almost always occurs in the direction at which the velocity pulse is observed.

3. FN direction and maximum direction angle, $\theta_{m}$, coincide (within $\pm 5^{\circ}$ ) for 40 percent of the records having velocity pulses. For records with apparent velocity pulses, this finding 
indicates that 60 percent of the time, maximum spectral acceleration takes place in directions other than the FN direction.

4. For a given GM pair, rotation angle, $\theta_{x}$, may alter the maximum pulse period significantly (for example GM 6); showing that the pulse period of rotated components varies with $\theta_{x}$.

\section{Description of Structural Systems and Computer Models}

The test-bed systems used are the 3D computer models of single-story and multistory symmetric- and asymmetric-plan buildings. Descriptions of their structural systems and computer models are discussed below.

\section{Single-Story Symmetric- and Asymmetric-Plan Buildings}

The first set of test-bed structures are 30 single-story buildings with three-degrees-offreedom. Their vibration periods, $T_{n}$, are equal to $0.2,1,2,3$, and 5 seconds. The yield strength reduction factors, $R$, are equal to 3,5 , and a value that leads to linear-elastic design. The lateralload resisting system of the buildings consists of buckling-restrained braced frames with nonmoment-resisting beam-column connections. The plan shapes and bracing layouts are shown in figure 8 . The buildings are identified by the letters A and B depending on the plan shape; plan A is rectangular with two axes of symmetry (torsionally stiff), whereas plan B is asymmetric (torsionally flexible) about both the $\mathrm{x}$ and $\mathrm{y}$ axes. The design spectrum was taken as the geometric mean of the 5 percent damped spectral acceleration response spectra of the FN components of the records (fig. 3). The earthquake design forces were determined by bidirectional linear response spectrum analysis (RSA) of the building with the design spectrum reduced by a response modification factor $R$.

The constitutive model used for the buckling restrained braces (BRBs) is the simplified trilinear model shown in figure 9. This model was obtained based on experimental results (Merritt and others, 2003). The parameters $k$ and $q_{y}$ are the same for all BRBs of a building. Plots of mode shapes and effective modal masses presented in figures 10 and 11 permit the following observations:

- Lateral displacements dominate motion of the A-plan (symmetric-plan) buildings in modes 1 and 2, whereas torsion dominates motion in the third mode. This indicates weak coupling between lateral and torsional components of motion. Additionally, the period of the dominantly torsional mode is much shorter than the period of the dominantly lateral modes, a property representative of buildings with lateral-load resisting systems located along the perimeter of the plan.

- Coupled lateral-torsional motions occur in the first and third modes of the B-plan (asymmetric plan) buildings, whereas lateral displacements dominate motion in the second mode. According to the ASCE/SEI 7-05 (ASCE, 2006), plan B presents an extreme torsional irregularity on the basis of difference between the center of gravity and center of rigidity on plan.

- The higher-mode contributions to response are expected to be significant for the B-plan buildings because the effective mass of the first lateral modes is less than 40 percent of the total mass. 


\section{Multistory Symmetric- and Asymmetric-Plan Buildings}

The second set of test-bed structures is nine-story symmetric- and asymmetric-plan buildings. The symmetric-plan structure is an existing steel building, located in Aliso Viejo, California (fig. 12). Its west elevation and the plan of floors 3 to 8 are shown in figure 13. The lateral load resisting system consists of two ductile steel moment frames in the longitudinal and transverse directions (fig. 13B); all structural members are standard I-sections, and the typical floors are made-up of 3 inch metal deck with 3-1/4 inch thick light weight concrete fill. The building façade consists of concrete panels and glass (fig. 12B), and there is a heliport on the roof (fig. 13A). Designed as an office building according to 2001 California Building Code for seismic zone 4 and National Earthquake Hazard Reduction Program (NEHRP) soil profile D, the earthquake forces were determined by linear RSA for the code design spectrum reduced by a response modification factor of 8.5.

Analyzed by the PERFORM-3D computer program (Computers and Structures, Inc. (CSI), 2006), the building was modeled as follows:

- Beams and columns were modeled by a linear element with trilinear plastic hinges at the ends of the elements that can include in-cycle strength deterioration but not cyclic stiffness degradation; the beam stiffness was modified to include the effect of the slab, and the axial load-moment interaction for the columns was based on plasticity theory;

- The braces below the heliport were modeled using fiber sections to model buckling behavior;

- Panel zones were modeled as four rigid links hinged at the corners with a rotational spring that represents the strength and stiffness of the connection;

- The tab connections were modeled using rigid perfectly plastic hinges that can include incycle and cyclic degradation;

- The contribution of nonstructural elements was modeled by adding four shear columns located close to the perimeter of the building, with their properties obtained from simplified models of the façade and partitions; nonlinear behavior of these elements was represented using rigid-plastic shear hinges;

- Ductility capacities of girders, columns, and panel zones were specified according to the ASCE/SEI 41-06 (ASCE, 2007) standard;

- Columns of moment resisting frames and the gravity columns were assumed to be clamped at the base; and

- Effects of geometric nonlinearity were approximated by a standard $\mathrm{P}-\Delta$ formulation.

Figure 14 shows the location and orientation of the 15 motion sensors (accelerometers)

installed in the building. The 2008 magnitude $\left(M_{w}\right) 5.4$ Chino-Hills earthquake-centered at a distance of $40 \mathrm{~km}$ - did not cause any observable damage, and reliable data were recorded by all eight sensors in the $y$ direction, but only by sensors 9 and 13 in the $x$ direction. The acceleration records indicate that the peak acceleration of $0.026 \mathrm{~g}$ at the ground was amplified to $0.042 \mathrm{~g}$ at the roof of the building (fig. 15).

Vibration properties — natural periods, natural modes and modal damping ratios—of the building were determined from recorded motions shown in figure 15 by applying two systemidentification methods. These are the combined deterministic-stochastic subspace (DSS) method (Van Overshee and De Morre, 1996) and the peak-picking (PP) method. Remarkably close agreement between the calculated (from the computer model) and identified values of vibration periods and modes from the recorded motions was achieved. Table 2 demonstrates the periods identified by both methods, and the natural vibration modes identified by the DSS method are 
presented in figure 16. As expected, the translational and torsional motions of this symmetricplan building are uncoupled (figs.17 and 18). The DSS method also led to the modal damping ratios of 4.3, 3.3, 3.96, and 3.22 percent for the first, third, fourth, and sixth modes of vibration. Based on these data, energy dissipation in the building was modeled by Rayleigh damping, with its two constants selected to give 4.3 percent and 3.96 percent damping ratio at the vibration periods of the first and fourth modes, respectively. The resulting damping ratios for the first nine vibration modes of the buildings ranged from 3.7 percent to 7.4 percent.

The computer model accurately represented the properties of the building as demonstrated by the excellent agreement between the computed and recorded responses. RHA of the computer model subjected to the recorded ground motion led to the displacements (relative to the ground) at floors 2, 5, 6, and 9 shown in figure 19, where these computed responses were compared with the motions recorded by sensors $6,8,10,11,14$, and 15 (see fig. 14); the agreement between the two is very good.

The asymmetric-plan structure considered is a nine-story steel building with ductile frames designed according to the 1985 Uniform Building Code (UBC85), which allows for significant plan irregularity. The plan shape and frame layout are shown in figure 20, where moment resisting frames are highlighted in the plans. The building has a span length of 30 feet and story height 13 feet with a plan asymmetry about both $x$ and $y$ axes. Design code forces for the building, assumed to be located in Bell, California $\left(33.996^{\circ} \mathrm{N}, 118.162^{\circ} \mathrm{W}\right)$, were determined, but their member sizes were governed by drift instead of strength requirements.

The building was modeled for nonlinear RHA, implemented in the 3D computer program, with the following features:

- Beams and columns were modeled by a linear element with trilinear plastic hinges at the ends of the elements that include in-cycle strength deterioration but not cyclic stiffness degradation (fig. 21). Axial load-moment interaction in columns is represented by plasticity theory;

- Panel zones were modeled as four rigid links hinged at the corners with a rotational spring that represents the strength and stiffness of the connection (fig. 22) (Krawinkler, 1978);

- Ductility capacities of girders, columns, and panel zones were specified according to the ASCE/SEI 41-06 standard (ASCE, 2007);

- At the base level, columns of moment resisting frames were assumed to be fixed, whereas gravity columns were considered pinned;

- The geometric nonlinear effects were considered by a standard $\mathrm{P}-\Delta$ formulation for both moment and gravity frames; and

- Damping was modeled by Rayleigh damping, with its two constants selected to give 2percent damping ratio at the fundamental period of vibration, $T_{1}$, and a period of $0.2 T_{1}$. The damping ratios for the first nine vibration modes range from 1.5 to 2.5 percent.

Figure 23 shows the natural periods and modes of vibration; $x_{c m}$ denotes the distance from the center of mass (C.M.) to a corner of the building. Figure 24 shows the motion of the roof in plan. The effective modal masses for the first nine modes of vibration are presented in figure 25. These figures indicate that torsional rotations dominate motion in the first and fourth modes, whereas lateral displacements dominate motion in the second and fifth modes, indicating weak coupling between lateral and torsional motions for these two sets of modes. Because the periods of the dominantly torsional modes are longer than those of the dominantly lateral modes, this system is said to be "torsionally flexible." Also, the higher-mode contributions to response are 
expected to be significant because the effective mass of the first lateral mode is less than 50 percent of the total mass.

\section{Evaluation Methodology}

The following steps were implemented for evaluating the significance of the groundmotion rotation angle on linear-elastic and nonlinear inelastic behavior of single-story and multistory buildings with symmetric- and asymmetric-plan located in near fault sites:

1. For each of the 30 records selected for this investigation, calculate rotated ground-motion components by varying $\theta_{x}$ from $0^{\circ}$ to $360^{\circ}$ at every $5^{\circ}$ in the clockwise direction (fig. $2 B$ ). The motions for $\theta_{x}=0^{\circ}$ and $90^{\circ}$ correspond to the FP and FN components of the record, respectively. In addition, calculate rotated ground-motion components for $\theta_{x}=\theta_{m}$ and $\theta_{x}=\theta_{m}+90^{\circ}$. For estimating maximum direction angle $\theta_{m}$ for single-story systems, use periods equal to $0.2,1,2,3$, and $5 \mathrm{~s}$, and for multistory structures, use fundamental periods of the buildings.

2. Calculate the 5-percent damped response spectrum $\mathrm{A}(T)$ for the $\mathrm{FN}$-component of the 30 records at 300 logarithmically spaced periods $T$ over the period range from 0.001 to 6 seconds.

3. Implement an iterative procedure for designing the 30 single-story systems described previously using the median spectrum of 30 FN components of step 2 as the design spectrum. At the end of this step, values for the parameters $k$ and $q_{y}$ are obtained for each $\mathrm{BRB}$. Recall that the single-story systems have vibration periods, $T_{n}$, equal to $0.2,1,2,3$, and 5 seconds and yield strength reduction factors $R$ equal to 3, 5, and a value that leads to linear-elastic design.

4. Conduct linear and nonlinear RHAs of the 30 single-story symmetric- and asymetric-plan systems subjected to bidirectional rotated components of ground motion obtained in step 1. For each RHA, obtain floor displacements, floor total accelerations, BRB plastic deformations, and BRB forces. This step involves more than 34,000 RHAs.

5. Conduct linear and nonlinear RHAs of the multistory buildings subjected to bidirectional, rotated components of ground motions obtained in step 1. For each RHA, obtain floor displacements, floor total accelerations, total chord rotations, and moments of beams and columns. This step involves 2,400 RHAs.

\section{Results}

Linear and nonlinear RHAs were implemented for the single-story and multistory systems of this investigation subjected to two horizontal components of ground motions following the aforementioned procedure. First, the results for the single-story systems will be presented.

\section{Single-Story Symmetric- and Asymmetric-Plan Buildings}

Selected EDPs for single-story systems are displacement $\left(u_{x},\right)$ and floor total acceleration $\left(\ddot{u}_{x}^{t}\right)$ at the center of mass, member force, and plastic deformation of selected BRBs. Figure 26 shows roof displacement, $u_{x}$, (red line) as a function of the rotation angle, $\theta_{x}$, for symmetric-plan buildings with $T_{n}=2,3$, and 5 seconds subjected to ground motions from table 1 with velocity 
pulse period close to $T_{n}$. The filled gray area shows values of $\theta_{x}$ in which the velocity pulses are identified for each record. Note that angles $\theta_{x}=0^{\circ}$ and $90^{\circ}$ correspond to the FP and FN directions, respectively. Figure 27 displays $\ddot{u}_{x}^{t}$ at the roof level. The member force and plasticdeformation at bracing b1 (fig. 8) are shown in figures 28 and 29, respectively. For asymmetricplan systems, roof displacements, $u_{x}$, at the center of mass and at the corner c2 (fig. 8) as a function of the rotation angle, $\theta_{x}$, are shown in figures 30 and 31, respectively. Figure 32 displays the roof total acceleration, $\ddot{u}_{x}^{t}$, at corner c2. The member forces for bracing b3 (fig. 8) as a function of $\theta_{x}$ are shown in figures 33 and 34, respectively. These figures permit the following observations:

- Velocity pulses may appear in directions different than the FN or FP direction.

- For symmetric-plan systems, the maximum displacement, $u_{x}$, and floor total acceleration, $\ddot{u}_{x}^{t}$, over all nonredundant orientations are generally polarized in the direction in which an apparent velocity pulse with a period close to $T_{n}$ is observed; although this polarization is almost perfect for linear-elastic systems, it vanishes for nonlinear-inelastic systems, and leading maximum displacement $u_{x}$ also occurs in the direction different from that of the velocity pulse (white areas in figs. 26-27 and 30-31); this is attributed to period elongation as a result of inelastic action. For asymmetric-plan systems, however, no strong correlation is observed between the orientation leading to maximum $u_{x}$ and $\ddot{u}_{x}^{t}$ and the velocity pulse direction even for linear-elastic response.

- Only for linear-elastic symmetric-plan systems, the maximum force in selected BRBs is polarized in the direction in which the pulse is identified. Whereas for all nonlinear-inelastic systems, BRB reaches its ultimate capacity quickly without being influenced by the rotation angle (fig. 28).

- For symmetric-plan systems, maximum values of EDPs almost always occur in the direction at which the velocity pulse is identified. For the asymmetric-plan systems, however, maximum values of EDPs also take place in directions without the velocity pulse (fig. 29).

- EDPs may be underestimated by more than 50 percent if a building is subjected to only FN/FP components of a pulse-like ground motion. This is valid for both symmetric- and asymmetric-plan systems.

- There is no unique orientation for a given structure; the rotation angle that leads to maximum EDPs varies not only with the ground-motion pair selected but also with the period, $R$, value used in the design process of the building and EDP of interest.

For a selected earthquake scenario, it is commonly assumed that EDPs are log-normally distributed (Cornell and others, 2002). For this reason, it is more appropriate to represent the "mean" structural response by the median; a conclusion that is widely accepted. Because the geometric mean and median of a random variable having a lognormal distribution are the same, we decided to employ the term "median" instead of geometric mean, as is commonly done. Figure 35 shows median displacements, $u_{x}$, at the center of mass as a function of the rotation angle, $\theta_{x}$, for symmetric-plan buildings with $T_{n}=0.2,1,2,3$, and $5 \mathrm{sec}$, and with $R=3$, 5 , and a value that leads to linear-elastic design subjected to 30 bidirectional ground motions. The red lines represent the median displacement, $u_{x}$, plus or minus one standard deviation $(\sigma)$ computed based on peak response values resulting from each ground-motion pair at each nonredundant rotation angle. In these figures, the blue circles represent the median MD displacement, $u_{m x} \pm \sigma$, (16th and 84th percentile values are compute as $u_{m x} \exp ( \pm \sigma)$ ) for the systems subjected to ground motions only in the MD. Recall that MD stands for maximum direction. Note that for a given ground-motion pair, MD changes with period. In figure 35, although the MD-displacement 
$u_{m x} \pm \sigma$ values correspond to a single value for each system, it is visualized as a full circle to facilitate direct comparisons with median displacements, $u_{x} \pm \sigma$, which is a function of the rotation angle, $\theta_{x}$. Similarly, median values of other EDPs are shown in figure 36 thru figure 37 for the symmetric-plan systems. For the asymmetric-plan systems, similar plots are depicted in figures 38 thru 41. These figures provide an overall statistical examination to generalize the observations previously made based on individual records in figures 26 thru 34. These general observations are:

- For short period ( $T_{n}=0.2$ seconds) linear-elastic symmetric- and asymmetric-plan systems, maximum median-displacement values (red lines) are independent of the ground-motion rotation angle. At longer periods, however, maximum median displacements are influenced by the rotation angle, and they are generally polarized with the FN direction. This is more pronounced for symmetric-plan systems. For $R$ values of 3 and 5, the effect of the rotation angle on displacement is significant for all systems.

- Median values of floor total accelerations and member forces are generally not influenced by the ground-motion rotation angle in both linear-elastic and nonlinear-inelastic range for both symmetric- and asymmetric-plan buildings.

- For all systems, it is clear that the $R$ value used in the design process affects the difference between the median MD displacement and the maximum median displacement over all nonredundant orientations. For linear-elastic systems, maximum values of EDPs are almost always smaller than median MD-EDPs—a conclusion drawn by Huang and others, (2008). However, for nonlinear-inelastic systems, maximum median-EDPs may be equal or larger than MD-EDPs. This is an important finding because it demonstrates that use of MD ground motions does not necessarily provide the most critical (or over conservative ${ }^{6}$ ) EDPs for systems responding in nonlinear-inelastic range in particular for asymmetric-plan structures.

- It is evident that conducting nonlinear RHA for ground motions oriented in the FN/FP directions does not always lead to the peak value of median-displacement over all nonredundant rotation angles. However, displacements are not underestimated substantially (less than 20 percent) if the system is subjected to only the FN/FP components of a large set of ground motions. The underestimation could be as much as 50 percent if a single groundmotion record (not a suite of records) is used for RHA.

\section{Multistory Symmetric- and Asymmetric-Plan Buildings}

Similar to the single-story systems, linear and nonlinear RHAs were implemented for the multistory structures subjected to bidirectional excitations following the aforementioned evaluation procedure. Figure 42 shows linear-elastic response quantities (story drifts, floor total accelerations, and beam and column moments) in $x$ direction at the 1st, 3rd, 5th, 7th, and 9th floors for the 9-story symmetric-plan building ( $T_{1}=1.51$ seconds) as a function of the rotation angle $\theta_{x}$ subjected to ground motion no. 9 in table 1 , which has a maximum velocity pulse period of 1.9 seconds. The filled gray area shows values of $\theta_{x}$ in which the velocity pulses are identified. The angles $\theta_{x}=0^{\circ}$ and $90^{\circ}$ correspond to the FP and FN directions, respectively. The record with pulse period close to the fundamental period of the building is selected intentionally, because such records impose sudden and intense energy input associated with the velocity pulse that should be dissipated within a short period of time. This causes amplified deformation demands in structures (Kalkan and Kunnath, 2006, 2007). Figure 42 indicates that maximum

\footnotetext{
${ }^{6}$ The term “conservative” is used here for either peak or close to peak EDP values.
} 
values of EDPs generally take place in the same direction, different than the FN direction, with the exception of the 9th floor $x$-column moment. Also, the maximum EDPs are observed in the direction in which the velocity pulse is identified. For this particular record, FN direction doesn't contain an apparent velocity pulse, and the EDPs in the FN direction are as much as 20 percent less than their maxima.

For a given response quantity of interest and record pair, the FN/FP directions will correspond to two values. By comparing these two values with the responses at all other possible rotation angles, one can evaluate the level of conservatism in such directions; for example, whether the FN/FP rotated ground motions provide an envelope of EDP. If obvious systematic benefits of the FN/FP orientations existed, they should be observable by repeating such comparisons for several EDPs and record pairs. To do this, figures 43 thru 46 show height-wise distribution of the median and dispersion values of EDPs plotted separately in $x$ and $y$ directions. In these plots, grey lines represent GMs rotated by $10^{\circ}$ increments. The continuous red line is for FN direction and the dashed red line is for FP direction. The blue line represents the components oriented to MD. Note that each line corresponds to either median or dispersion of RHA results of 30 ground-motion pairs rotated by $\theta_{x}$.

These figures present interesting results. While the ground motions rotated to the FN direction yield the largest median EDPs in the $x$ direction, the motions oriented to FP direction yield the largest median-EDPs in the $y$ direction, thus EDPs due to the FN/FP rotated ground motions serve as envelopes for all other nonredundant rotation angles. Note that the $x$ direction of the building coincides with $0^{\circ}$ (FP direction). As opposed to the linear-elastic results based on single-story systems, ground motions rotated to MD produce smaller median-EDPs as compared those due to FN/FP directions. Dispersions of EDPs are also larger in the FN/FP directions than in the MD.

The results plotted in figures 43 thru 46 are consolidated and depicted as a function of $\theta_{x}$ in figures 47 and 48 for $x$ and $y$ directions of the building, respectively. Viewing the response as a function of rotation angle enables us to better understand how the critical angle, $\theta_{c r}$, defined as the angle corresponding to the largest response over all angles, varies with both EDP and ground-motion pair. For this building $\theta_{c r}=0^{\circ}$ and $90^{\circ}$ because the maximum median EDPs along the $x$ direction are polarized in the $\mathrm{FN}$ direction (that is, $90^{\circ}$ ), and the maximum median EDPs along the $y$ direction are polarized in the FP direction (that is, $0^{\circ}$ ). It is evident that maximum median EDPs are generally larger than the median MD-EDPs in both $x$ and $y$ directions indicating that MD ground motions do not necessarily provide the most critical (or over conservative) EDPs for systems responding in linear-elastic range.

Same response quantities are plotted in figures 49 thru 55 for the linear 9-story asymmetric-plan building ( $T_{1}=2.5$ seconds). As opposed to the symmetric-plan building, it is

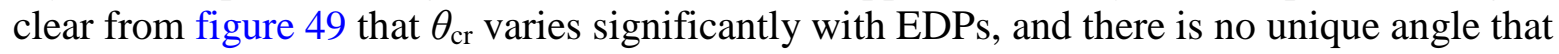
leads to the peak values for all EDPs simultaneously. Examination of height-wise distributions of median and dispersion of EDPs in figures 50 thru 53 demonstrate that neither the FN/FP directions nor the MD rotated ground motion produce the maximum median drift and internal forces in the $x$ direction. In the $y$ direction, however, ground motions rotated to FN/FP direction led to the maximum median drift and floor acceleration. For other EDPs in the same direction, arbitrary orientations resulted in maximum median values. For almost all EDPs, the maximum median values that corresponded to the MD were smaller than those corresponded to the FN/FP direction. This finding is also evident in figure 54 and figure 55, where the median $\pm \sigma$ values of EDPs are plotted as a function of $\theta_{x}$. These figures once again demonstrate that the maximum 
median EDPs (solid red line) are dependent on the rotation angle of the ground motion and that it varies with EDP. For the asymmetric-plan building, maximum median-EDPs resulting from the MD rotated ground motions are generally smaller than those resulting from the ground motion rotated to the FN/FP direction. This is more pronounced for EDPs in the $y$ direction. Similar to the symmetric-plan building, the MD rotated ground motions do not necessarily provide the most critical results for the asymmetric-plan building.

So far linear-elastic response quantities were examined for the symmetric- and asymmetric-plan 9-story buildings. In the next set of figures, we will examine the nonlinearinelastic response quantities. Nonlinear results for the symmetric-plan building are shown first in figures 56 thru 62. Similar plots for the asymmetric-plan building are depicted in figures 63 thru 69. It is evident that $\theta_{\text {cr }}$ leading to maximum response varies significantly with EDPs for nonlinear - systems. While the FN/FP direction rotated ground motions yield the largest value for certain EDPs, there is no single $\theta_{\text {cr }}$ that lead to the peak values for all EDPs. Note that the same conclusion drawn for linear-elastic systems. Unlike the symmetric-plan building, peak values of EDPs also take place in the direction in which no apparent pulse is identified (for example, $x$ column moment at 9th floor). Examination of height-wise distributions of median and dispersion values of EDPs also demonstrates important findings. The ground motions rotated to the MD result in the maximum median EDPs in the $x$ direction, whereas same records surprisingly produce the minimum median EDPs in the $y$ direction, in which the ground motions oriented to the FP direction yield the largest median EDPs. This observation is consistent for all EDPs investigated for both symmetric- and asymmetric-plan buildings.

Ground motions rotated to the FN direction produce the second largest median EDPs following the results associated with the MD. Therefore, for nonlinear-inelastic response, the MD and FP direction rotated ground motions serve as envelopes for all other nonredundant rotation angles. These observations are also evident in figures 61-62 and 68-69, where the median $\pm \sigma$ values of EDPs are plotted as a function of $\theta_{x}$. For nonlinear-inelastic systems, these figures prove that the maximum median EDPs (solid red lines) are dependent on the rotation angle of the ground motion only for certain EDPs; for example, member forces and plastic rotations are not affected by the rotation angle as much as the drift does. For both symmetricand asymmetric-plan buildings, the maximum median EDPs resulting from the MD rotated ground motions yield conservative results only for the $x$ direction of the buildings, whereas in the other direction ground motions oriented to FP direction provide the most conservative results. Thus, no consistency in over conservatism of MD rotated ground motions is detected. These findings are in close agreement with those derived based on the linear-elastic systems.

\section{Conclusions}

Current seismic design practice in the United States requires as-recorded pairs of ground motions to be rotated to fault-normal and fault-parallel (FN/FP) directions before they are used as input for three-dimensional (3D) response history analyses (RHAs) of building structures within $5 \mathrm{~km}$ of the active fault. It is assumed that this approach will lead to two sets of responses that envelope the range of possible responses over all nonredundant rotation angles. Thus, it is considered to be a conservative method appropriate for design verification of new structures. Additionally, the site-specific ground-motion procedure, according to the ASCE/SEI 7-10, requires that the ground motion to be rotated to the maximum direction (MD) (that is, direction of rotated ground-motion pair resulting in peak linear-elastic response quantity of a single lumped mass oscillator) when site-response analysis is performed; this new approach has been 
found to be controversial (Stewart and others, 2011). Currently, there is a lack of research addressing bidirectional nonlinear response of structures considering ground-motion directionality effects. In this study, the influence that the rotation angle of the ground motion has on several engineering demand parameters (EDPs) has been examined systematically in linearelastic and nonlinear-inelastic domains using a suite of 3D computer models of symmetric- and asymmetric-plan single-story and multistory buildings subjected to 30 bidirectional near-fault ground motions. Also investigated are the rotation angle of an apparent velocity pulse and its correlation with the MD and FN/FP directions.

This investigation has led to the following conclusions:

- Velocity pulses in near-fault records may appear in directions different than the MD or FN/FP directions. For the near-fault records examined, MD shows large scattering with no visible correlation with the FN/FP directions; this is valid even for motions recorded within 5 $\mathrm{km}$ of the fault.

- For linear-elastic systems, the maximum displacement occurs when in the direction where apparent velocity pulse with a period close to the fundamental period of the structure is observed. This strong polarization vanishes for nonlinear-inelastic systems due to period elongation. For other EDPs (for example, member forces), their linear and nonlinear peak values are generally independent of the ground-motion rotation angle. These observations are valid for both symmetric- and asymmetric-plan single-story and multistory buildings investigated.

- There is no unique orientation for a given structure maximizing all EDPs simultaneously; maximum EDP can happen in any direction different than the direction of the velocity pulse. The critical angle $\theta_{c r}$ corresponding to the largest response over all possible rotation angles varies with the ground-motion pair selected, $\mathrm{R}$ value used in the design process and the response quantity (EDP) of interest. Therefore, it is difficult to determine a "unique" building orientation that maximizes demands for all EDPs before conducting a RHA.

- For a given record, the rotation angle leading to maximum elastic response is different than that resulting in maximum inelastic response; therefore, there is no single rotation angle that operates effectively in both linear and nonlinear domains.

- For a given ground-motion pair, MD is not unique; it changes with period and $R$ value of the system, as a result, the MD response spectrum becomes an envelope of the maximum response spectral accelerations of the ground-motion pair at all possible rotation angles and periods. It is therefore argued that the use of MD ground motion for design is an overly conservative approach. Although it can be true for linear-elastic systems, conducting nonlinear RHA for ground motions oriented in the MD does not always lead to maximum EDPs over all orientations in particular for asymmetric-plan buildings.

- The use of MD or FN/FP directions applied along the structural axes of the building almost never guarantees that the maximum response over all possible angles will be obtained. Even though this approach may lead to a maximum value for one EDP, it will simultaneously be nonconservative for other EDPs. Therefore, if the performance assessment and design verification will be conducted against worst-case scenarios, then bidirectional ground motions should be applied at various angles with respect to the structural axes to cover all possible responses. Although this might not be a practical solution, it could still be worth conducting for certain projects.

- Treating the as-recorded direction as a randomly chosen direction, it is observed that there is more than a 50 percent chance for the larger response among the FN and FP values to exceed 
the response corresponding to an arbitrary orientation. The latter observation is valid for most but not all of the record pairs and response quantities considered. Therefore, compared to no rotation at all, use of the larger response of the two values corresponding to the MD or FN/FP directions is still warranted.

The results presented herein have important implications for current earthquake engineering practice, suggesting that ground motions rotated to MD or FN/FP directions do not necessarily provide the most critical estimates of EDPs in nonlinear-inelastic range. For a given record, rotation angle leading to maximum elastic response is different than that for maximum inelastic response, thus any conclusions drawn based on linear-elastic system will not be applicable for nonlinear-inelastic systems.

\section{Acknowledgments}

Juan C. Reyes would like to acknowledge the generous support of Universidad de los Andes (Colombia) and the U.S. Geological Survey for providing him the financial support for conducting this research. Finally, thanks to Andrew Whittaker, Yahya Kurama, David Boore, Charlie Kircher, C.B. Crouse, Katsuichiro Goda, and Sanaz Rezaeian for their critical reviews on the material presented herein and offering valuable comments and suggestions, which helped in improving the technical quality of this report.

\section{References Cited}

American Society of Civil Engineers, 2010, Minimum design loads for buildings and other structures, ASCE/SEI 7-10: Reston, Va., American Society of Civil Engineers, 650 p.

American Society of Civil Engineers, 2006, Minimum Design loads for buildings and other structures, ASCE Standard 7-05: Reston, Va., American Society of Civil Engineers, 424 p.

American Society of Civil Engineers, 2007, Seismic rehabilitation of existing buildings: Reston, Va., American Society of Civil Engineers, report no. 41-6, 411 p.

Athanatopoulou, A.M., 2004, Critical orientation of three correlated seismic components: Engineering Structures, v. 27, p. 301-312.

Baker, J.W., 2007, Quantitative classification of near-fault ground motions using wavelet analysis: Bulletin of the Seismological Society of America, v. 97, no. 5, p. 1486-1501.

Bray, J., and Rodriguez-Marek, A., 2004, Characterization of forward-directivity ground motions in the near-fault region: Soil Dynamics and Earthquake Engineering, v. 24, p. 815-828.

Campbell, K.W., and Bozorgnia, Y., 2007, Campbell-Bozorgnia NGA ground motion relations for the geometric mean horizontal component of peak and spectral ground motion parameters: Pacific Earthquake Engineering Research Center, University of California, Berkeley, report no. 2007-02, 238 p.

Computers and Structures, Inc., 2006, PERFORM 3D, user guide v4, nonlinear analysis and performance assessment for 3D structures: Berkeley, Calif., Computers and Structures, Inc.

Cornell, C.A., Jalayer, F., Hamburger, R.O., and Foutch, D.A., 2002, Probabilistic basis for 2000 SAC Federal Emergency Management Agency steel moment frame guidelines: Journal of Structural Engineering, v. 128, no. 4, p. 526-533.

Fernandez-Davila, I., Comeinetti, S., and Cruz, E.F., 2000, Considering the bidirectional effects and the seismic angle variations in building design: Proceedings of the 12th World Conference on Earthquake Engineering, Auckland, New Zealand.

Goda, K., 2012, Comparison of peak ductility demand of inelastic SDOF systems in maximum elastic response and major principal directions: Earthquake Spectra, v. 28, p. 385-399. 
Golesorkhi, R., and Gouchon, J., 2002, Near-source effects and correlation to recent recorded data: Proceedings of the 7th U.S. National Conference on Earthquake Engineering, Boston, Mass.

Hong, H.P., and Goda, K., 2010. Characteristics of horizontal ground motion measures along principal directions: Earthquake Engineering and Engineering Vibrations, v. 9, p. 9-22.

Huang, Y., Whittaker, A.S., and Luco, N., 2008, Maximum spectral demands in the near-fault region: Earthquake Spectra, v. 24, no.1, p. 319-34.

International Conference of Building Officials, 2009, International Building Code: Whittier, CA. International Conference of Building Officials, 2010, Whittier, Calif., California Building Code.

Kalkan, E., and Kunnath, S.K., 2006, Effects of fling-step and forward directivity on the seismic response of buildings: Earthquake Spectra, v. 22, no. 2, p. 367-390.

Kalkan, E., and Kunnath, S.K., 2007, Effective cyclic energy as a measure of seismic demand: Journal of Earthquake Engineering, v. 11, no. 5, p. 725-751.

Kalkan, E., and Kunnath, S.K., 2008, Relevance of absolute and relative energy content in seismic evaluation of structures: Advances in Structural Engineering, v. 11, no. 1, p. 17-34.

Kalkan, E., and Kwong, N.S., 2012, Evaluation of fault-normal/fault-parallel directions rotated ground motions for response history analysis of an instrumented six-story building: U.S. Geological Survey Open-File Report 2012-1058, 30 p., available at http://pubs.usgs.gov/of/2012/1058/.

Kalkan, E., and Kwong, N.S., in press, Pros and cons of rotating ground motion records to faultnormal/parallel directions for response history analysis of buildings: Journal of Structural Engineering.

Khoshnoudian, F., and Poursha, M., 2004, Responses of three dimensional buildings under bidirectional and unidirectional seismic excitations: Proceedings of the 13th World Conference on Earthquake Engineering, Vancouver, Canada.

Krawinkler, H., 1978, Shear in beam-column joints in seismic design of frames: Engineering Journal, v. 15, no. 3, p. 82-91.

Lagaros, N.D., 2010, Multicomponent incremental dynamic analysis considering variable incident angle: Structure and Infrastructure Engeering, v. 6, p. 77-94.

Lopez, O.A., and Torres, R., 1997, The critical angle of seismic rotation and structural response: Earthquake Engineering and Structural Dynamics, v. 26, p. 881-894.

Lopez, O.A., Chopra, A.K., and Hernandez, J.J., 2000, Critical response of structures to multicomponent earthquake excitation: Earthquake Engineering and Structural Dynamics, v. 29, p.1759-1778.

MacRae, G.A., and Mattheis, J., 2000, Three-dimensional steel building response to near-fault motions: Journal of Structural Engineering, v. 126, no.1, p.117-126.

Mavroeidis, G.P., and A.S. Papageorgiou, 2003, A mathematical representation of near-fault ground motions: Bulletin of the Seismological Society of America, v. 93, no. 3, p. 1099-1131.

Merritt S., Uang, C.M., and Benzoni, G., 2003, Subassemblage Testing of star seismic buckling restrained braces,report no. TR-2003/04; Final report to Star Seismic, LLC: University of California, San Diego.

Penzien, J., and Watabe, M., 1975, Characteristics of 3-dimensional earthquake ground motions: Earthquake Engineering and Structural Dynamics, v. 3, p. 365-373.

Rigato, A., and Medina, R.A., 2007, Influence of angle of rotation on the seismic demands for inelastic single-storey structures subjected to bidirectional ground motions: Engineering Structures, v. 29, no.10, p. 2593-2601. 
Singh, J.P., Porter L.D., and Zafir, Z., 2011, A practitioner's perspective of ASCE/SEI 7-10 maximum direction ground motions: Proceedings of the Annual Convention of Structural Engineers Association of California.

Stewart, J.P., Abrahamson, N.A., Atkinson, G.M., Baker, J., Boore, D.M., Bozorgnia, Y., Campbell, K.W., Comartin, C.D., Idriss, I.M., Lew, M., Mehrain, M., Moehle, J.P., Naeim, F., and Sabol, T.A., 2011, Representation of bidirectional ground motions for design spectra in building codes: Earthquake Spectra, v. 27, no. 3, p. 927-937.

Tezcan, S.S., and Alhan, C., 2001, Parametric analysis of irregular structures under seismic loading according to the new Turkish earthquake code: Engineering Structures, v. 23, p. 600609.

Van Overschee, P., and De Moor, B., 1996, Subspace identification for linear systems: Boston, London, and Dordrecht, Kluwer Academic Publishers.

Watson-Lamprey, J., and Boore, D.M., 2007, Beyond SaGMRotI—Conversion to SaArb, SaSN, and SaMaxRot: Bulletin of the Seismological Society of America, v. 97, p. 1511-1524.

Wilson, E.L., and Suharwardy, I., 1995, A clarification of the orthogonal effects in a threedimensional seismic analysis: Earthquake Spectra, v. 11, no.4, p. 659-666. 
Table 1. Selected near-fault ground-motion records

\begin{tabular}{|c|c|c|c|c|c|c|}
\hline $\begin{array}{l}\text { Record } \\
\text { sequence } \\
\text { number }\end{array}$ & Earthquake name & Year & Station name & $\begin{array}{l}\text { Earthquake } \\
\text { magnitude } \\
\left(M_{w}\right)\end{array}$ & $\begin{array}{l}\text { Style of } \\
\text { Faulting }\end{array}$ & $\begin{array}{l}\text { Closest fault } \\
\text { distance } \\
\text { (kilometers) }\end{array}$ \\
\hline 1 & Gazli, USSR & 1976 & Karakyr & 6.8 & Thrust & 5.5 \\
\hline 2 & Imperial Valley-06 & 1979 & Aeropuerto Mexicali & 6.5 & Strike-slip & 0.3 \\
\hline 3 & Imperial Valley-06 & 1979 & Agrarias & 6.5 & Strike-slip & 0.7 \\
\hline 4 & Imperial Valley-06 & 1979 & Bonds Corner & 6.5 & Strike-slip & 2.7 \\
\hline 5 & Imperial Valley-06 & 1979 & EC Meloland Overpass FF & 6.5 & Strike-slip & 0.1 \\
\hline 6 & Imperial Valley-06 & 1979 & El Centro Array \#6 & 6.5 & Strike-slip & 1.4 \\
\hline 7 & Imperial Valley-06 & 1979 & El Centro Array \#7 & 6.5 & Strike-slip & 0.6 \\
\hline 8 & Irpinia, Italy-01 & 180 & Auletta & 6.9 & Normal & 9.6 \\
\hline 9 & Irpinia, Italy-01 & 1980 & Bagnoli Irpinio & 6.9 & Normal & 8.2 \\
\hline 10 & Irpinia, Italy-01 & 1980 & Sturno & 6.9 & Normal & 10.8 \\
\hline 11 & Nahanni, Canada & 1985 & Site 1 & 6.8 & Thrust & 9.6 \\
\hline 12 & Nahanni, Canada & 1985 & Site 2 & 6.8 & Thrust & 4.9 \\
\hline 13 & Nahanni, Canada & 1985 & Site 3 & 6.8 & Thrust & 5.3 \\
\hline 14 & Superstition Hills-02 & 1987 & Parachute Test Site & 6.5 & Strike-slip & 1.0 \\
\hline 15 & Superstition Hills-02 & 1987 & Westmorland Fire Sta & 6.5 & Strike-slip & 13.0 \\
\hline 16 & Loma Prieta & 1989 & BRAN & 6.9 & Reverse & 10.7 \\
\hline 17 & Loma Prieta & 1989 & Gilroy Array \#3 & 6.9 & Reverse & 12.8 \\
\hline 18 & Loma Prieta & 1989 & LGPC & 6.9 & Reverse & 3.9 \\
\hline 19 & Loma Prieta & 1989 & San Jose - Santa Teresa Hills & 6.9 & Reverse & 14.7 \\
\hline 20 & Loma Prieta & 1989 & Saratoga - Aloha Ave & 6.9 & Reverse & 8.5 \\
\hline 21 & Loma Prieta & 1989 & Saratoga - W Valley Coll. & 6.9 & Reverse & 9.3 \\
\hline 22 & Erzican, Turkey & 1992 & Erzincan & 6.7 & Strike-slip & 4.4 \\
\hline 23 & Northridge-01 & 1994 & Jensen Filter Plant Generator & 6.7 & Reverse & 5.4 \\
\hline 24 & Northridge-01 & 1994 & Newhall - Fire Sta & 6.7 & Reverse & 5.9 \\
\hline 25 & Northridge-01 & 1994 & Newhall - W Pico Canyon Rd. & 6.7 & Reverse & 5.5 \\
\hline 26 & Northridge-01 & 1994 & Pacoima Dam (downstr) & 6.7 & Reverse & 7.0 \\
\hline 27 & Northridge-01 & 1994 & Rinaldi Receiving Sta & 6.7 & Reverse & 6.5 \\
\hline 28 & Northridge-01 & 1994 & Sylmar - Olive View Med FF & 6.7 & Reverse & 5.3 \\
\hline 29 & Kobe, Japan & 1995 & KJMA & 6.9 & Reverse & 1.0 \\
\hline 30 & Kobe, Japan & 1995 & Nishi-Akashi & 6.9 & Reverse & 7.1 \\
\hline
\end{tabular}


Table 2. Natural periods of vibration obtained from the peak-picking (PP) method, the combined deterministic-stochastic subspace (DSS) method, and the computer model for the ninestory symmetric-plan building.

$[-$, no data $]$

\begin{tabular}{|c|c|c|c|c|}
\hline \multirow{2}{*}{ Mode } & \multirow{2}{*}{ Direction } & \multicolumn{2}{|c|}{ Identified Period [sec] } & Computer Model \\
\cline { 3 - 4 } & & PP method & DSS method & \\
\hline 1 & translational $y$ & 1.58 & 1.53 & 1.51 \\
\hline 2 & translational $x$ & 1.46 & - & 1.45 \\
\hline 3 & torsional & 1.08 & 1.07 & 1.01 \\
\hline 4 & translational $y$ & 0.55 & 0.49 & 0.54 \\
\hline 5 & translational $x$ & 0.50 & - & 0.50 \\
\hline 6 & torsional & 0.38 & 0.36 & 0.36 \\
\hline
\end{tabular}

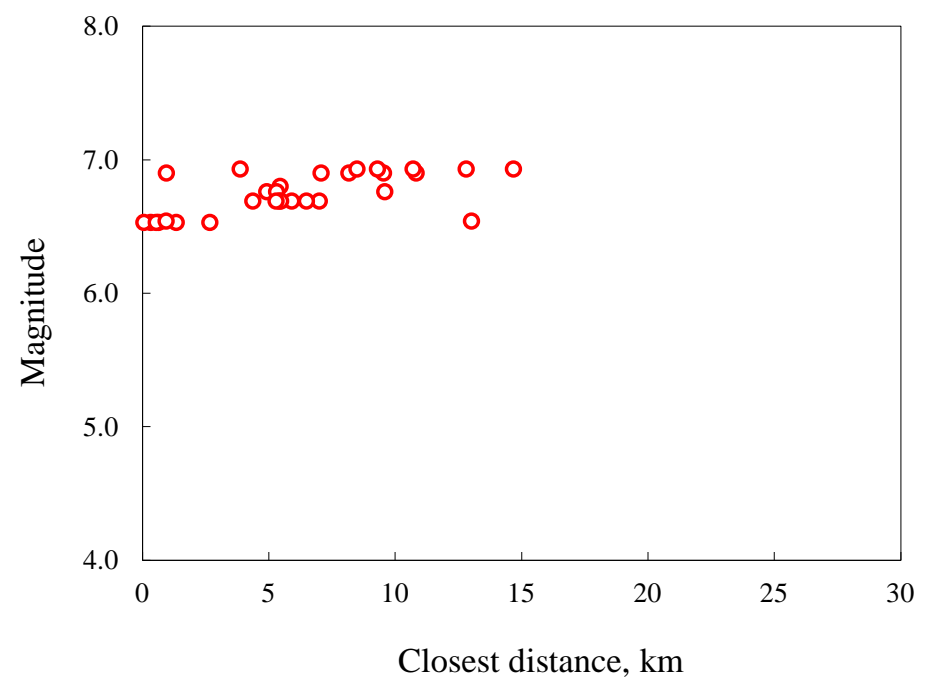

Figure 1. Distribution of magnitude $\left(M_{w}\right)$ and closest fault distance $\left(R_{\text {rup }}\right)$ for the 30 near-fault groundmotion records selected. 


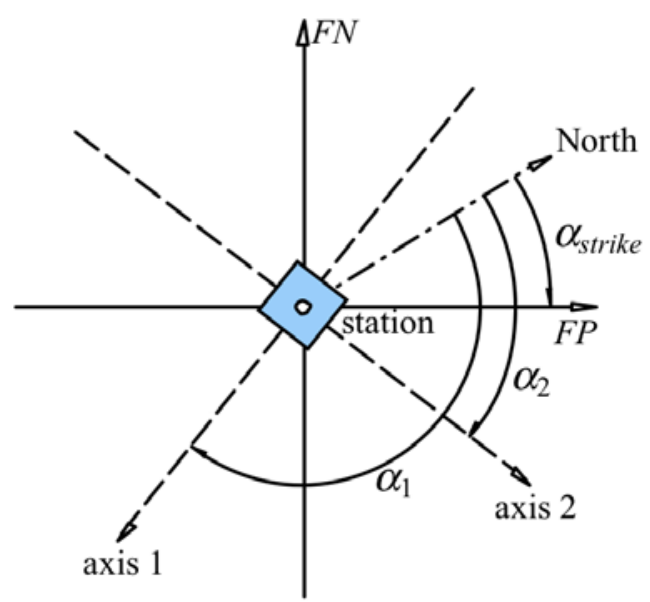

(A)

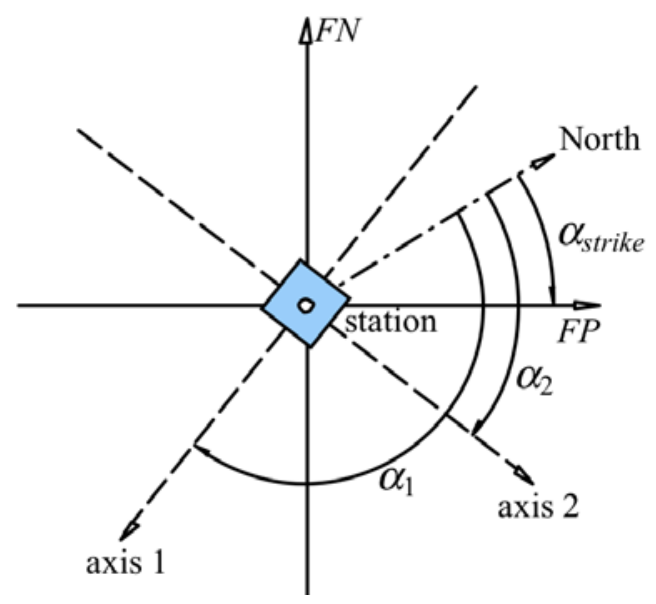

(A)

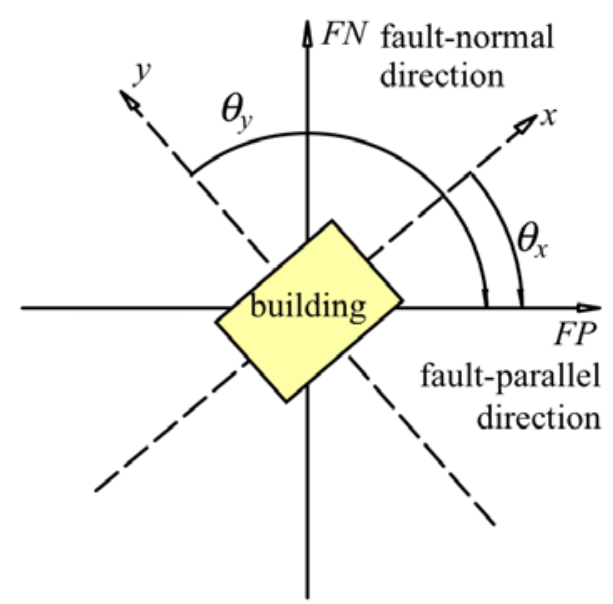

(B)

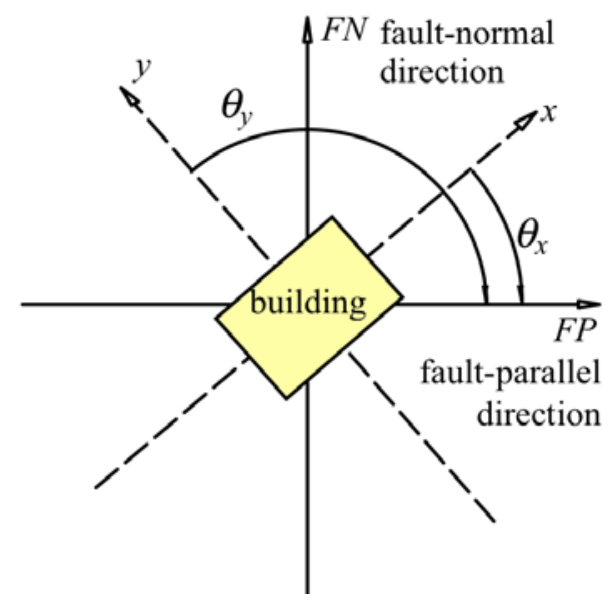

(B)

Figure 2. A, Reference axes for the fault and the instrument with relevant angles noted. $B$, Reference axis for the building. 

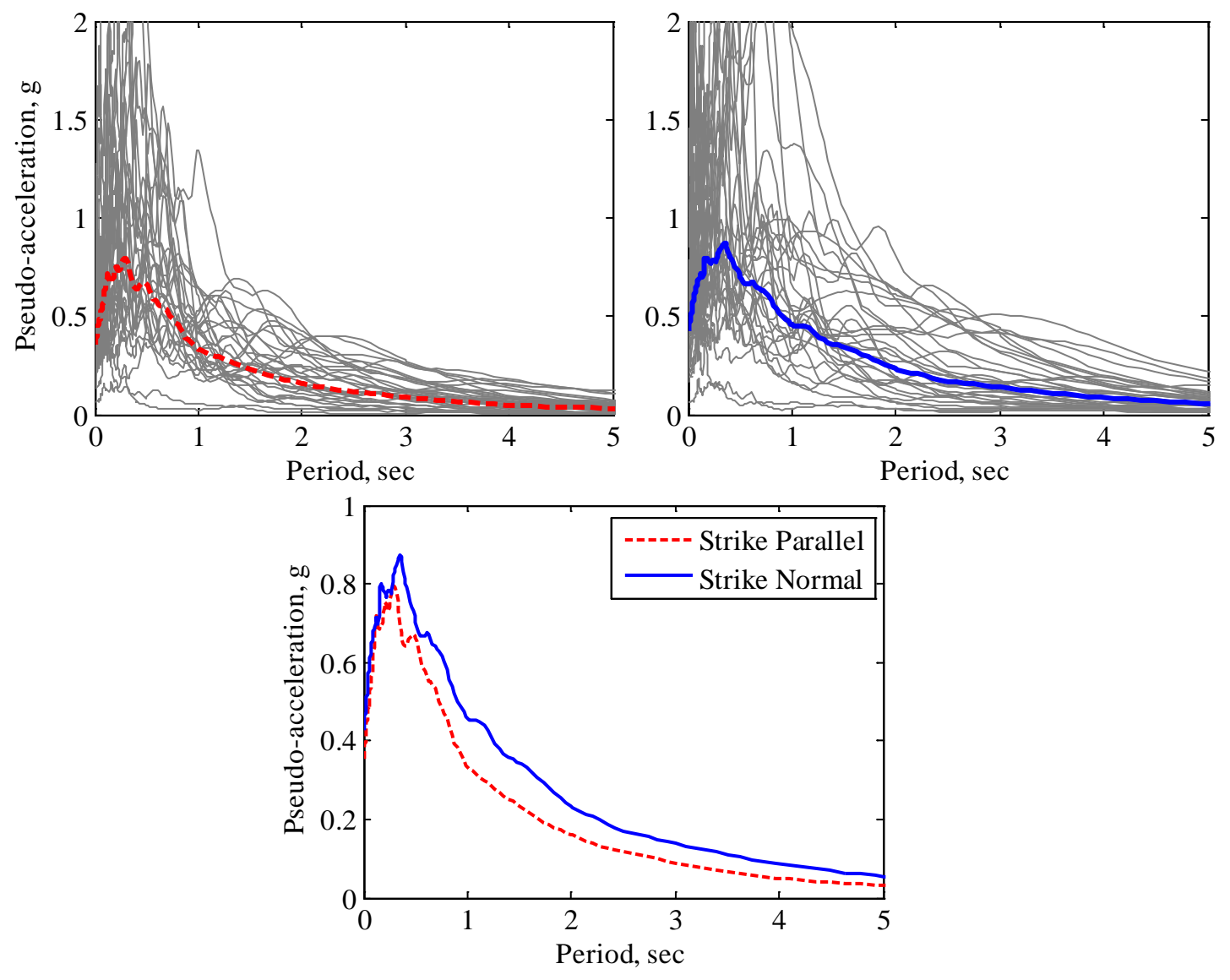

Figure 3. Response spectra of 30 near-fault ground motions and their geometric-mean used as design spectrum for single-story systems: fault parallel (strike parallel) components (left top panel), fault normal (strike normal) components (right top panel), comparison of median spectra of fault normal and fault parallel components (bottom panel). Damping ratio is 5 percent. $g$, acceleration due to gravity; sec, seconds. 


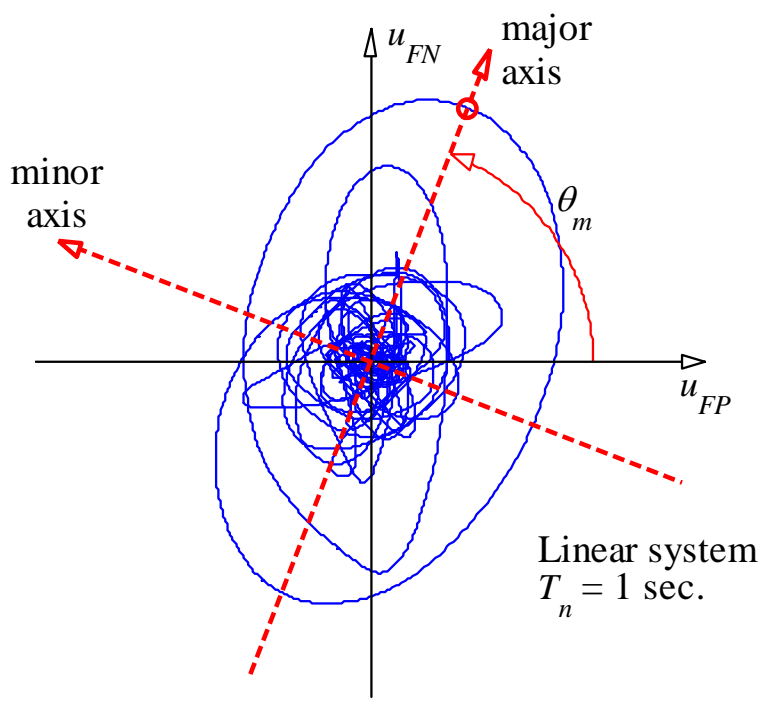

Figure 4. Trace of deformation $(u)$ orbit of a two-degrees-of-freedom system (elastic vibration period, $T_{n}=$ 1 second) with direction-independent stiffness and damping subjected to the fault-normal/fault parallel (FN/FP) components of a ground motion. 

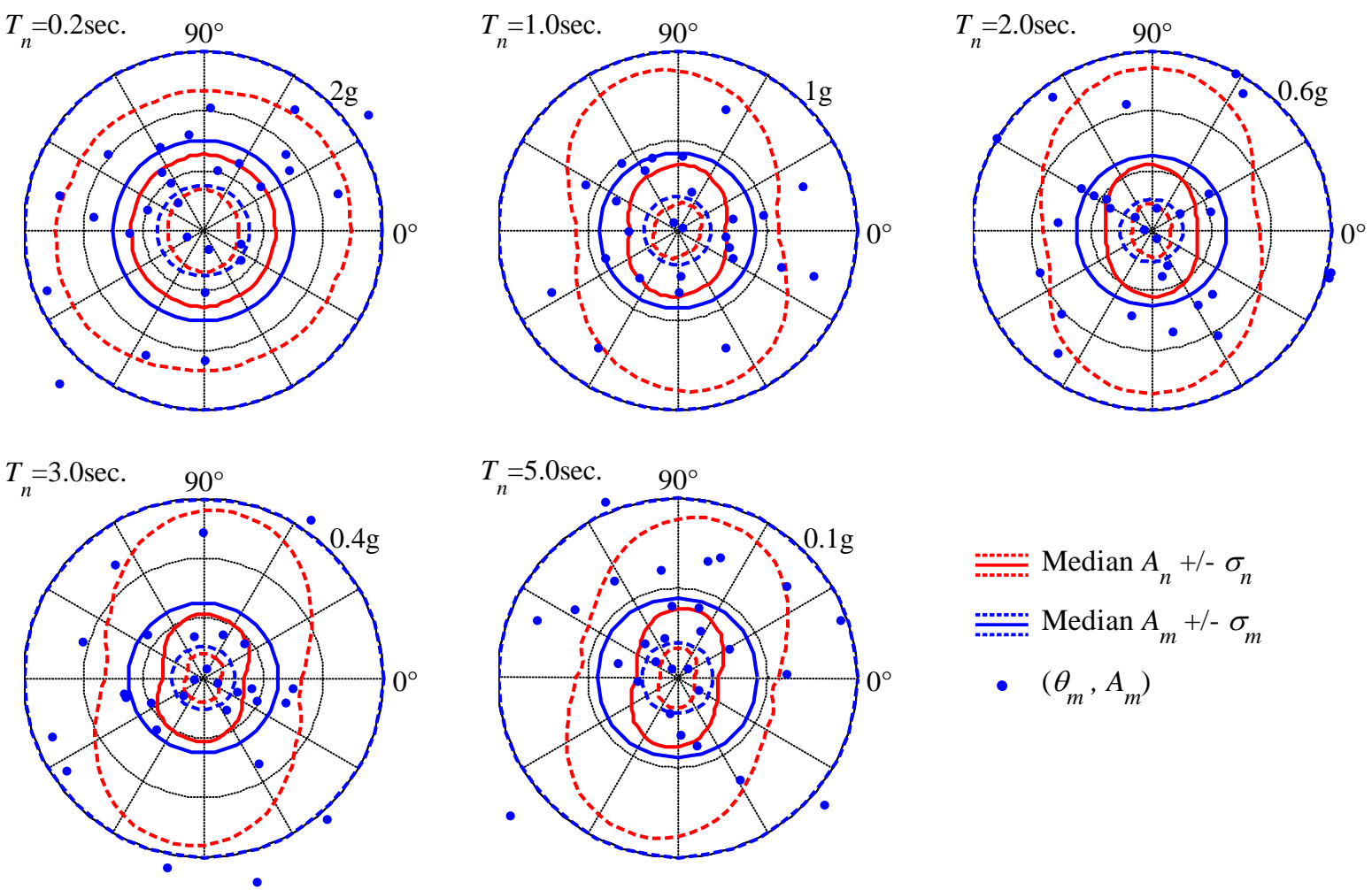

$$
\begin{aligned}
& \text {-..--.- Median } A_{n}+/-\sigma_{n} \\
& \text {-....... Median } A_{m}+/-\sigma_{m} \\
& \text { - }\left(\theta_{m}, A_{m}\right)
\end{aligned}
$$

Figure 5. For 30 near-fault ground-motion pairs listed in table 1, polar plots of spectral accelerations as a function of the rotation angle, $\theta_{x}$, are shown for elastic single-degree-of-freedom (SDF) systems with vibration period $\left(T_{n}\right)$ equal to $0.2,1,2,3$, and 5 seconds (damping ratio 5 percent). The red lines represent the median spectral acceleration value $\left(A_{n}\right) \pm$ one standard deviation $\left(\sigma_{n}\right)$. The blue points correspond to pairs of maximum direction angle $\theta_{m}$ and spectral acceleration values $A_{m}$. The blue circles represent the median spectral acceleration value $\pm \sigma_{m}$ in the maximum direction. Note that except for short-period SDF systems $\left(T_{n}=0.2\right.$ seconds), $A_{n}$ values are generally polarized with faultnormal $\left(90^{\circ}\right)$ direction; however, $\theta_{m}$ shows large scattering with no correlation with fault-normal direction; also spectral acceleration values, $A_{m}$, corresponding to the maximum direction angle, $\theta_{m}$, are generally higher than median spectral acceleration value, $A_{n}$. 

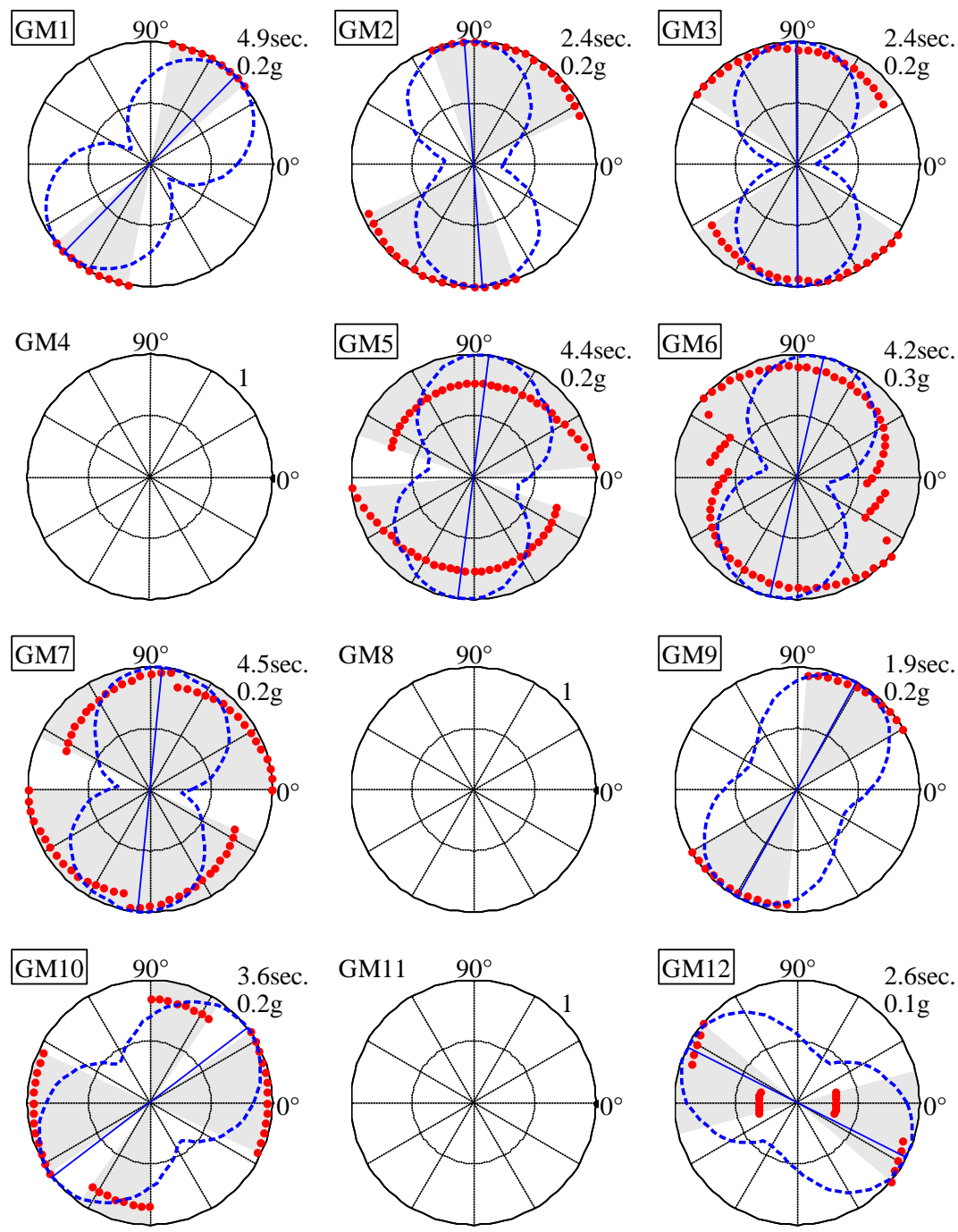

Direction and

- period of identified pulse-like motion

.... Spectral acc. for maximum pulse-period

Maximum-direction for maximum pulse-period
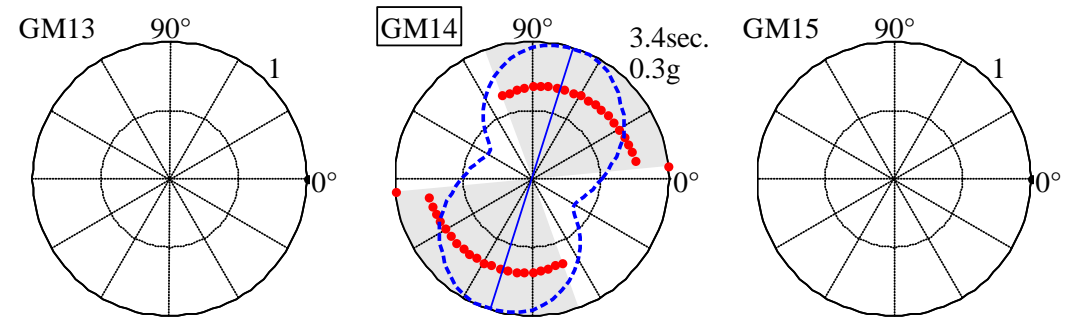

Figure 6. Polar plots of identified velocity pulse periods and spectral acceleration (acc.) values as a function of the rotation angle, $\theta_{x}$, for ground-motion (GM) pairs 1 to 15 (see table 1). The red dots show the directions in which velocity pulses are identified with their corresponding pulse periods. The filled gray area shows range of $\theta_{x}$ in which velocity pulses are observed. The dashed blue lines show spectral acceleration values for the maximum identified pulse period (damping ratio 5 percent). The blue line identifies the maximum direction. Numerical values for maximum pulse periods and maximum spectral accelerations are presented in the upper right corner of each subplot. Empty polar plot indicates that no velocity pulse is identified. 

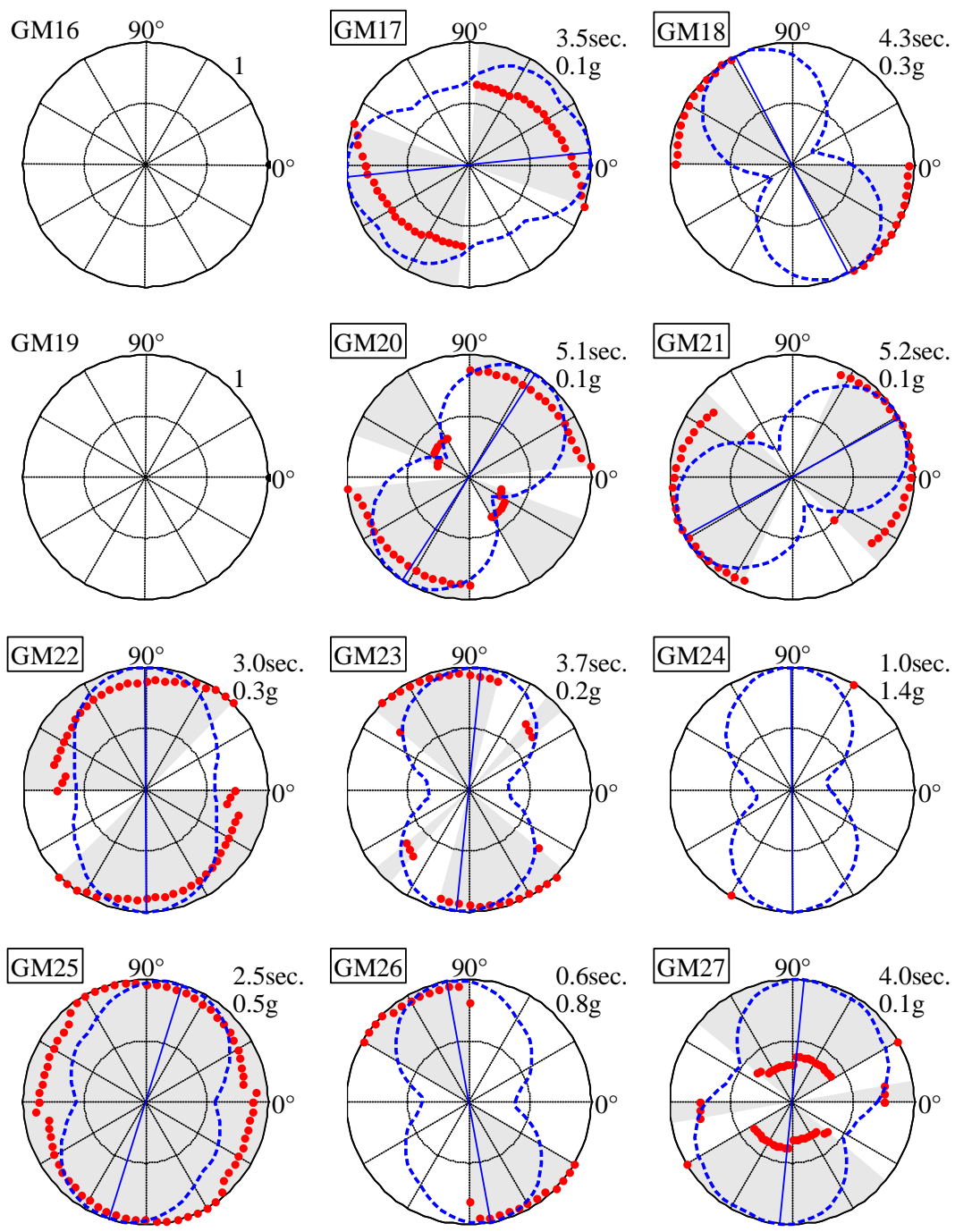

Direction and

- period of identified pulse-like motion

.... Spectral acc. for maximum pulse-period

$-\begin{aligned} & \text { Maximum-direction for } \\ & \text { maximum pulse-period }\end{aligned}$
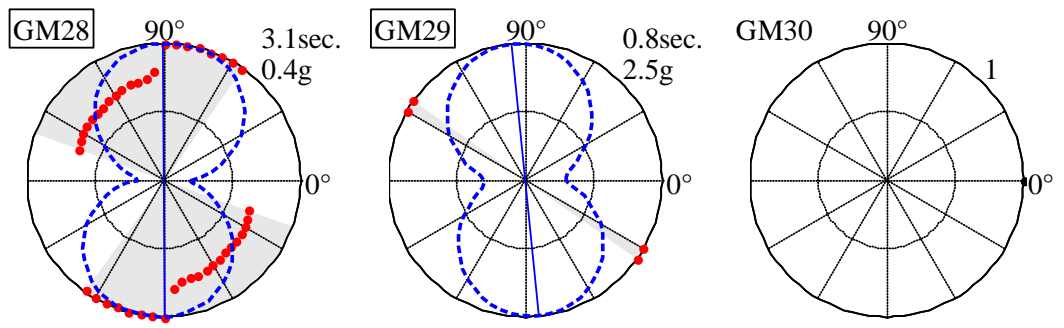

Figure 7. Polar plots of identified velocity pulse periods and spectral acceleration (acc) values as a function of the rotation angle, $\theta_{x}$, for ground-motion (GM) pairs 16 to 30 (see table 1). The red dots show the directions in which velocity pulses are identified with their corresponding pulse periods. The filled gray area shows range of $\theta_{x}$ in which velocity pulses are observed. The dashed blue lines show spectral acceleration values for the maximum identified pulse period (damping ratio 5 percent). The blue line identifies the maximum direction. Numerical values for maximum pulse periods and maximum spectral accelerations are presented in the upper right corner of each subplot. Empty polar plot indicates that no velocity pulse is identified. 
Plan A: torsionally-stiff buildings
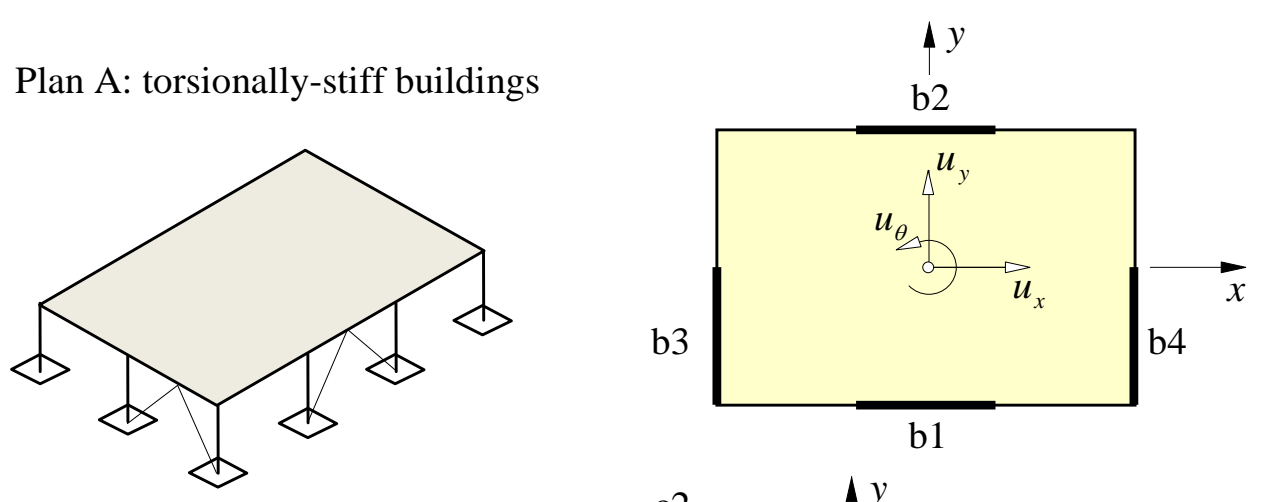

Plan B: torsionally-flexible buildings
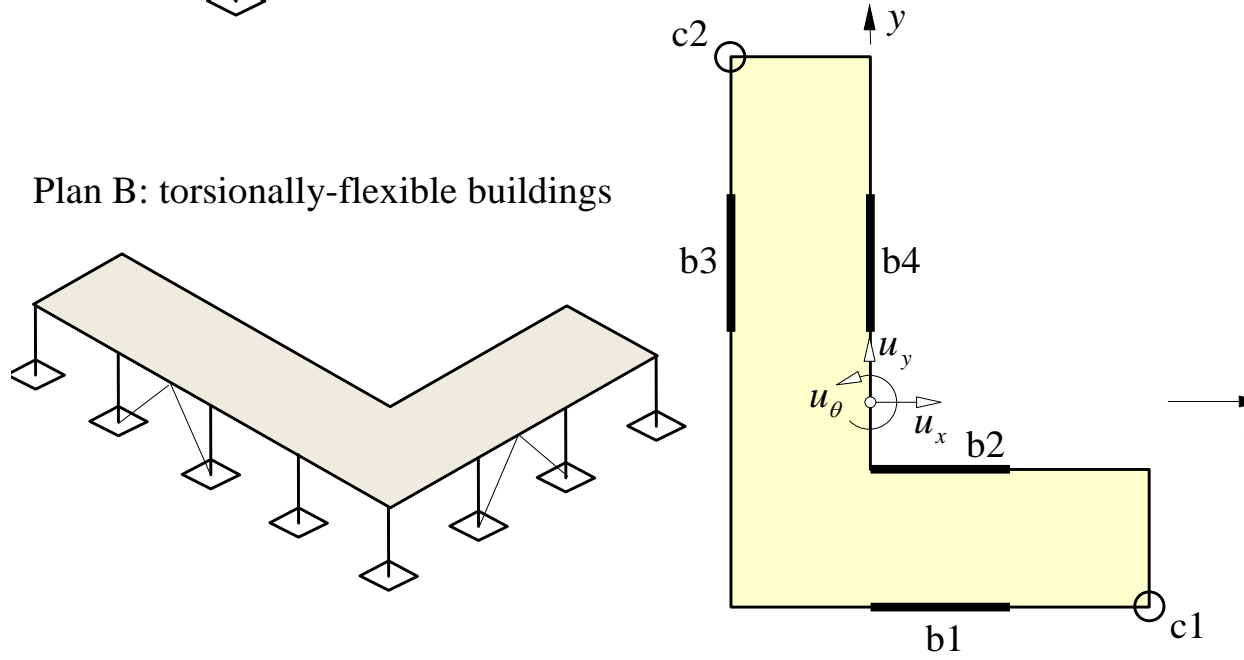

Figure 8. Schematic isometric and plan views of the selected single-story structural systems with threedegrees-of-freedom noted; buckling-restrained braced frames are highlighted. 


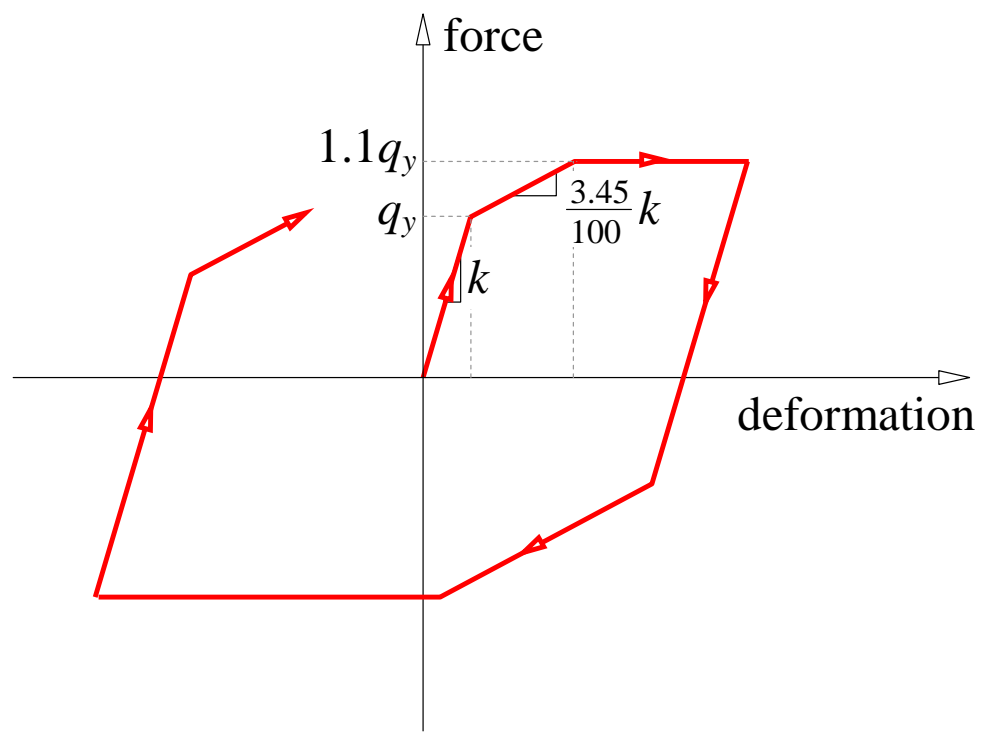

Figure 9. Constitutive model used for the buckling-restrained braces (BRBs).

Mode 1
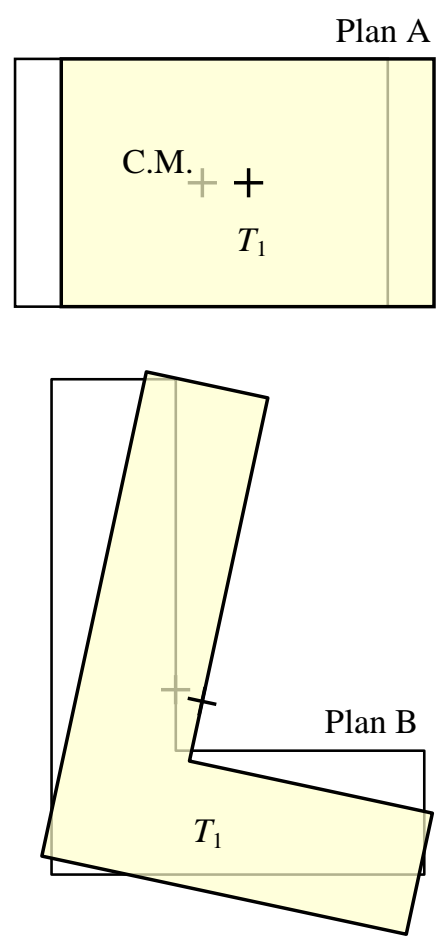

Mode 2
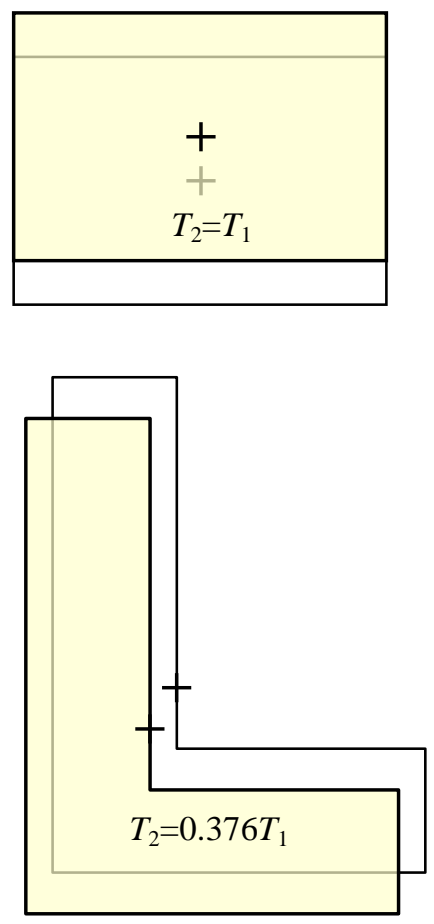

Mode 3
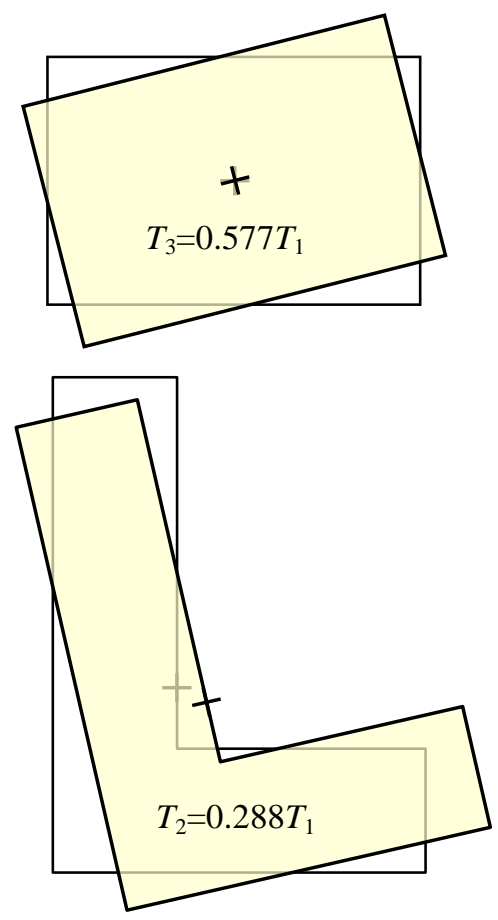

Figure 10. Periods and modes of vibration of the single-story three-degrees-of-freedom systems $\left(T_{n}=\right.$ elastic vibration period for the $n_{\text {th }}$ mode). 


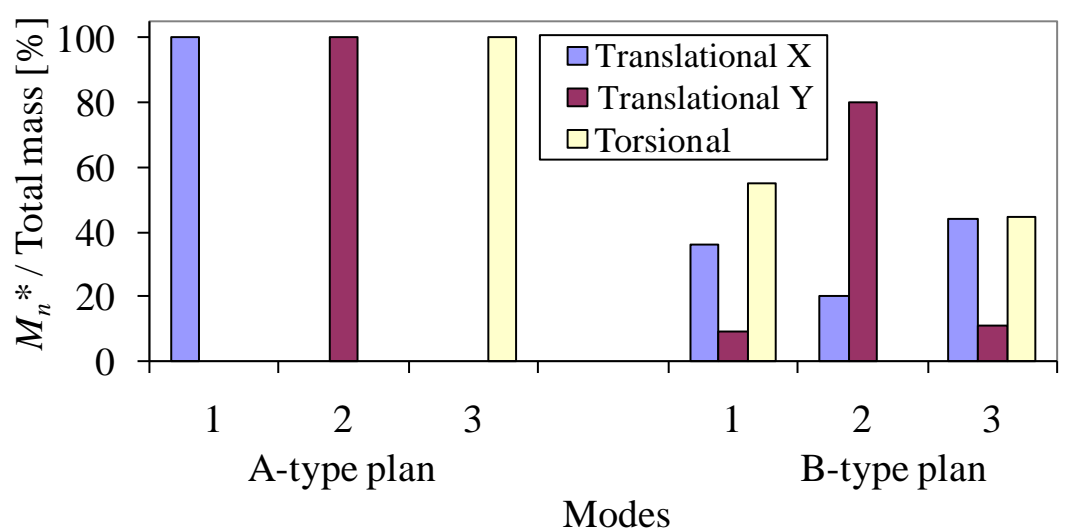

Figure 11. Effective modal masses, $M_{n}{ }^{*}$, of the plan-A (symmetric-plan) and plan-B (asymmetric-plan) buildings. \%, percent.

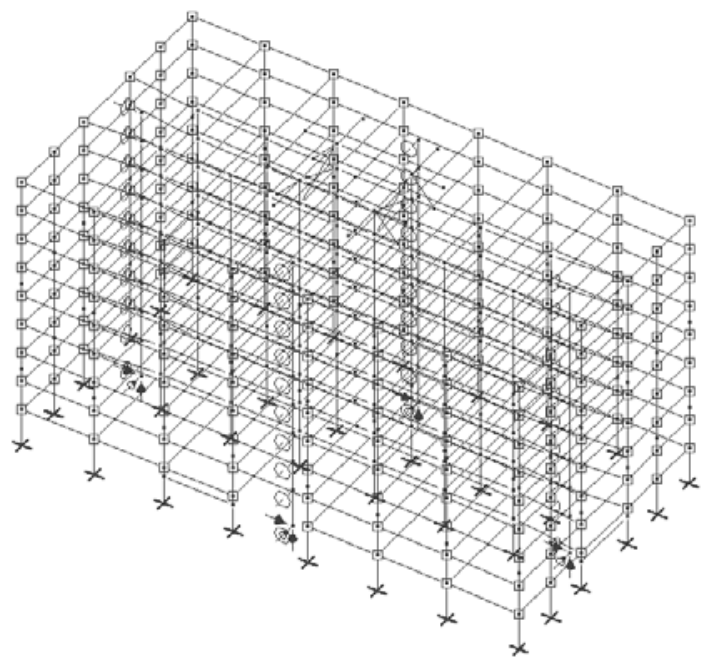

$A$

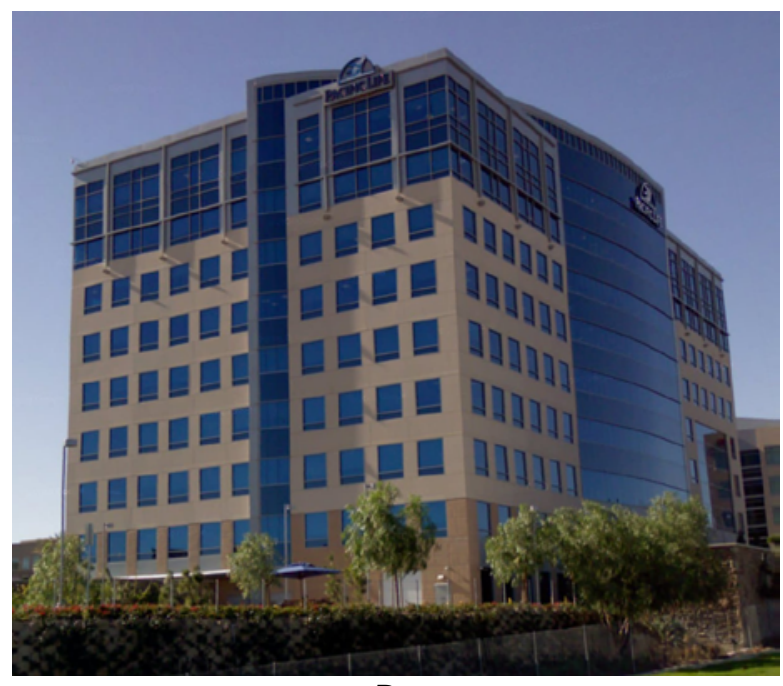

$B$

Figure 12. Nine-story symmetric-plan building in Aliso Viejo, California. A, three-dimensional computer model. $B$, Photograph of the building looking to north elevation. 

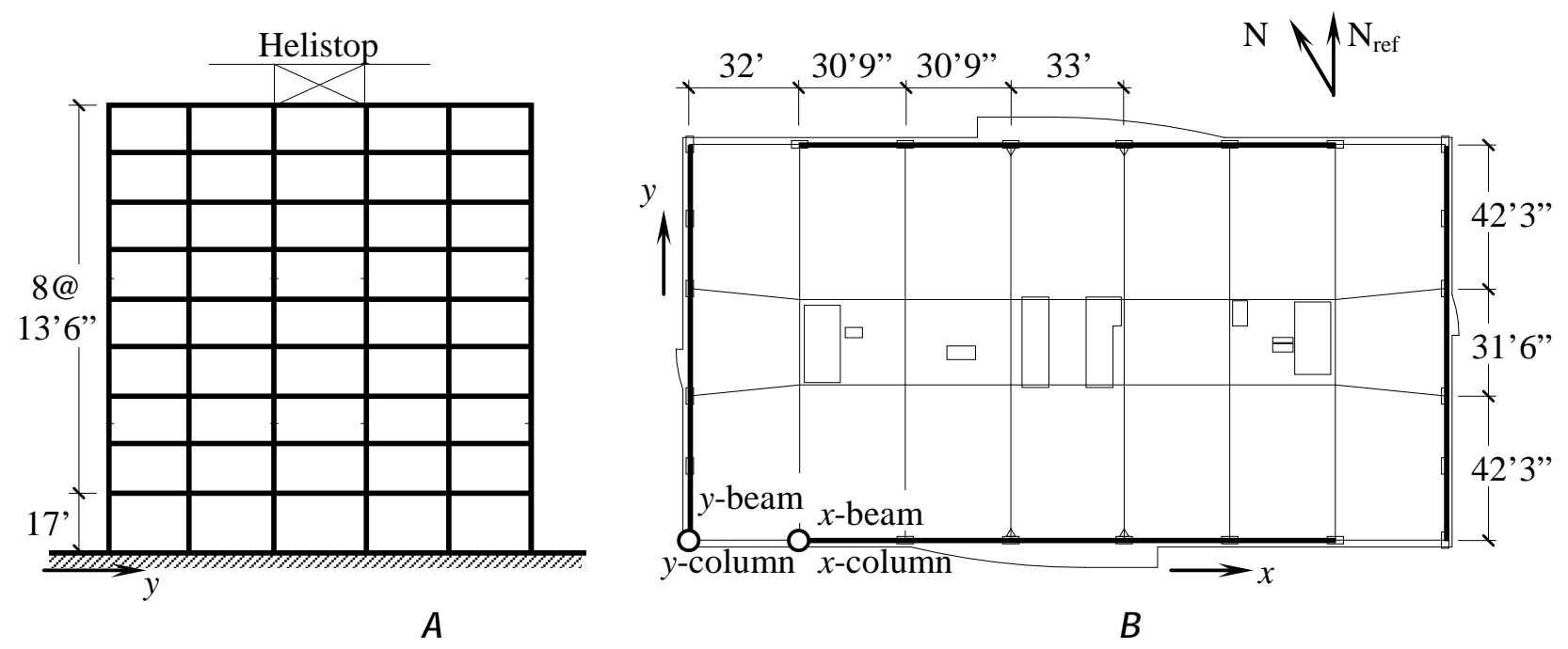

Figure 13. West elevation $(A)$ and typical floor plan $(B)$ of symmetric-plan building. 


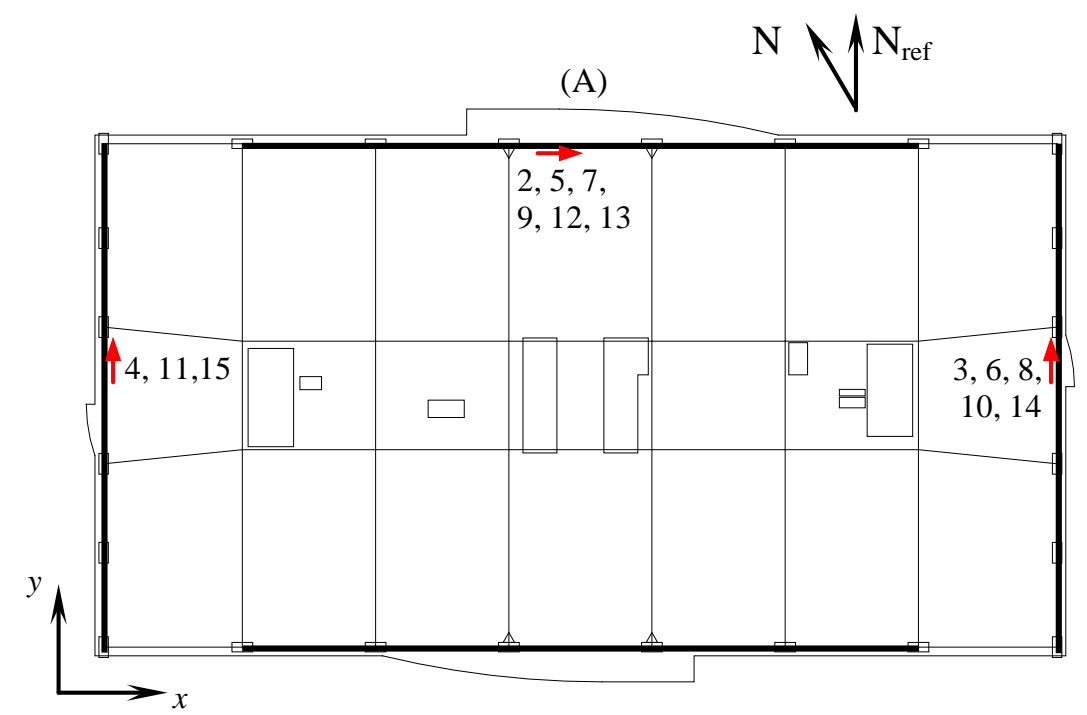

(B)

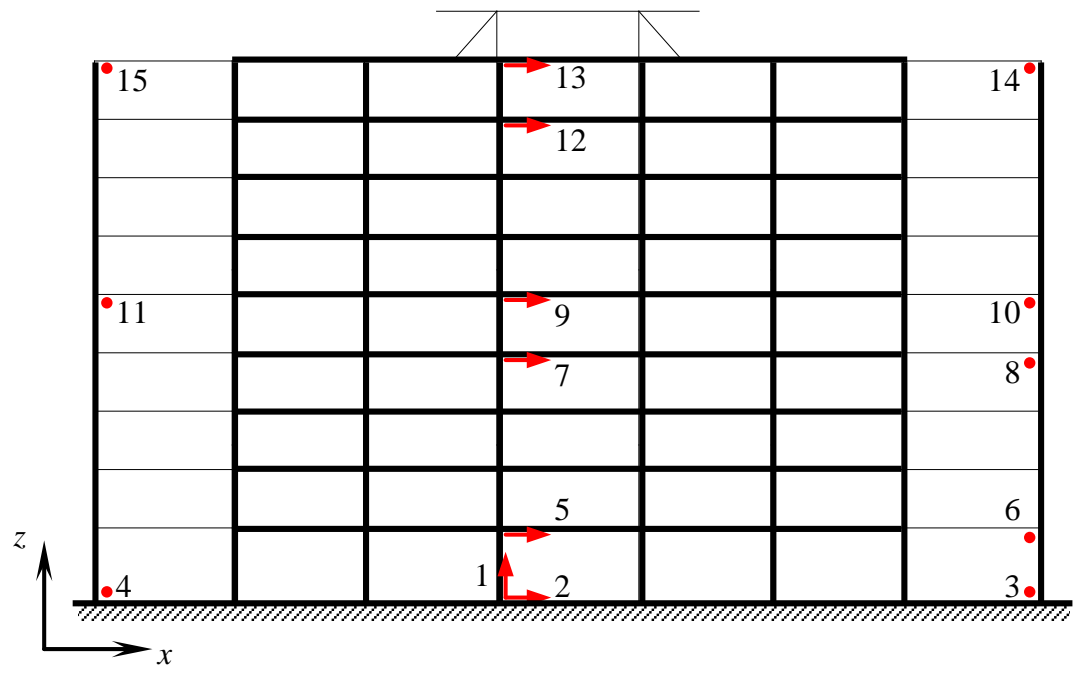

Figure 14. Locations of sensors shown with red arrows in symmetric-plan building. $A$, Plan view. $B$, south elevation. 

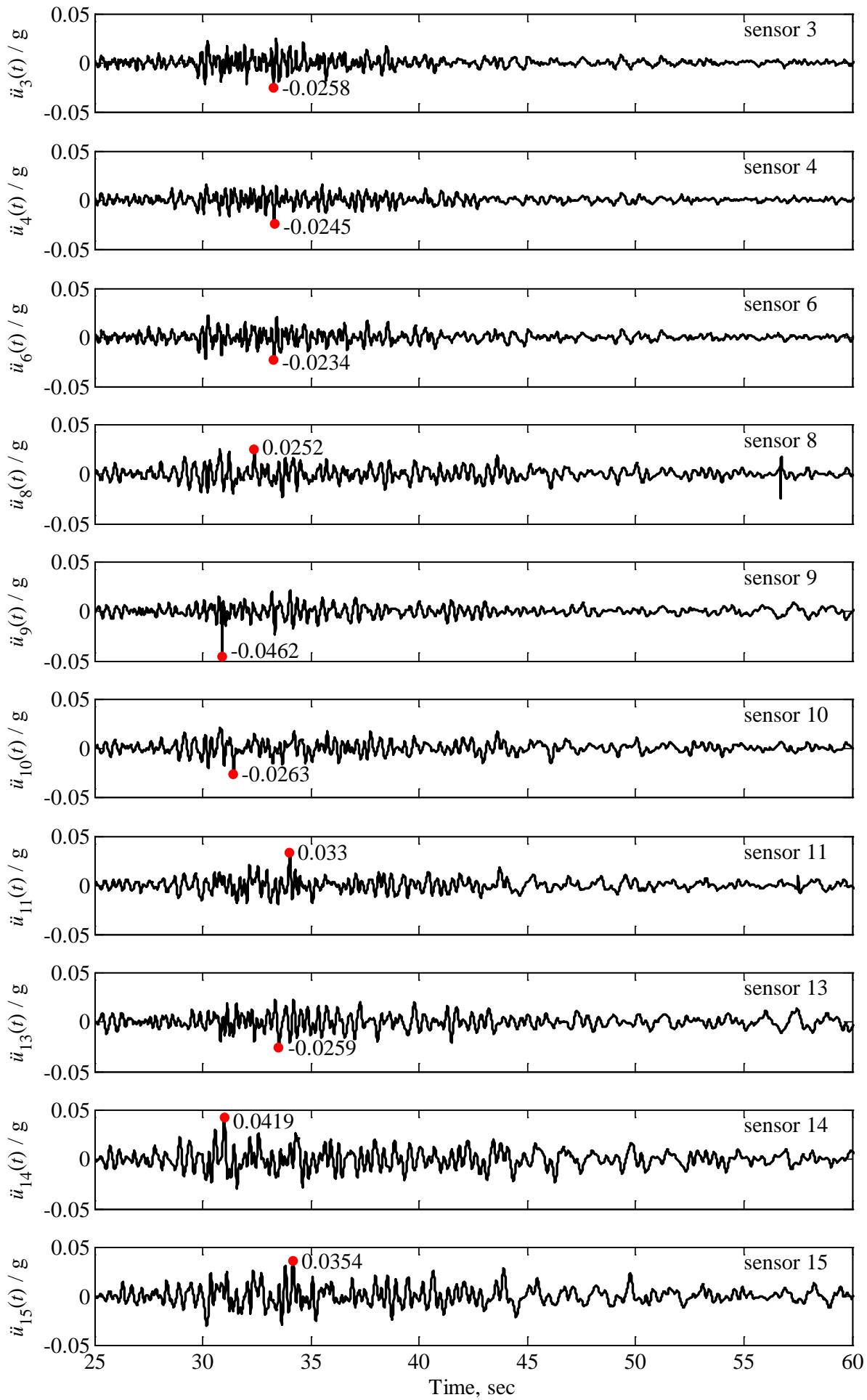

Figure 15. Floor total accelerations recorded by the sensors shown in figure 14 during the 2008 magnitude 5.4 Chino Hills southern California earthquake. 

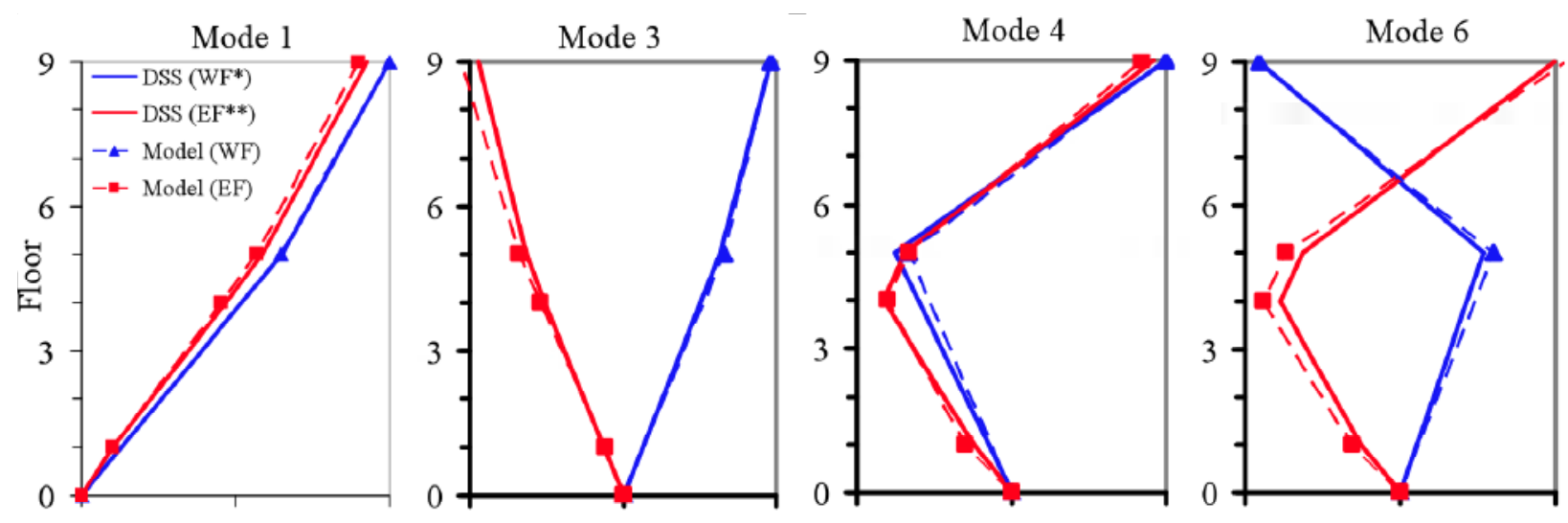

Figure 16. Comparison of normalized natural vibration modes identified by the deterministic-stochastic subspace (DSS) method with modes of the computer model for symmetric-plan building ( ${ }^{*} \mathrm{WF}=$ west frame; ** $\mathrm{EW}=$ east frame).

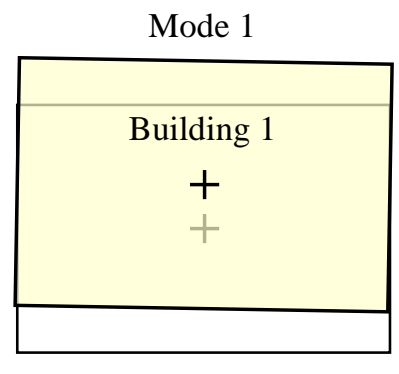

$T_{1}=1.51 \mathrm{sec}$.
Mode 2

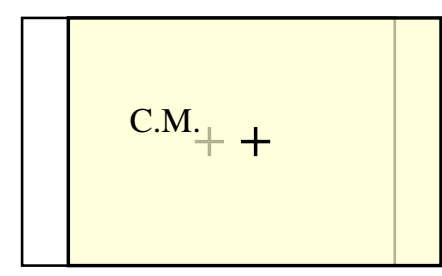

$T_{2}=1.45 \mathrm{sec}$.
Mode 3

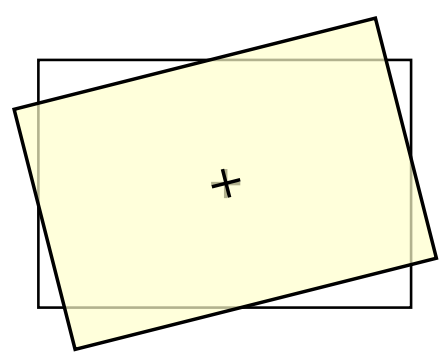

$T_{3}=1.01 \mathrm{sec}$.

Figure 17. First triplet of periods and modes of vibration of symmetric-plan building (only roof motion is shown), ( $T_{n}=$ elastic vibration period for the $n_{\text {th }}$ mode). 


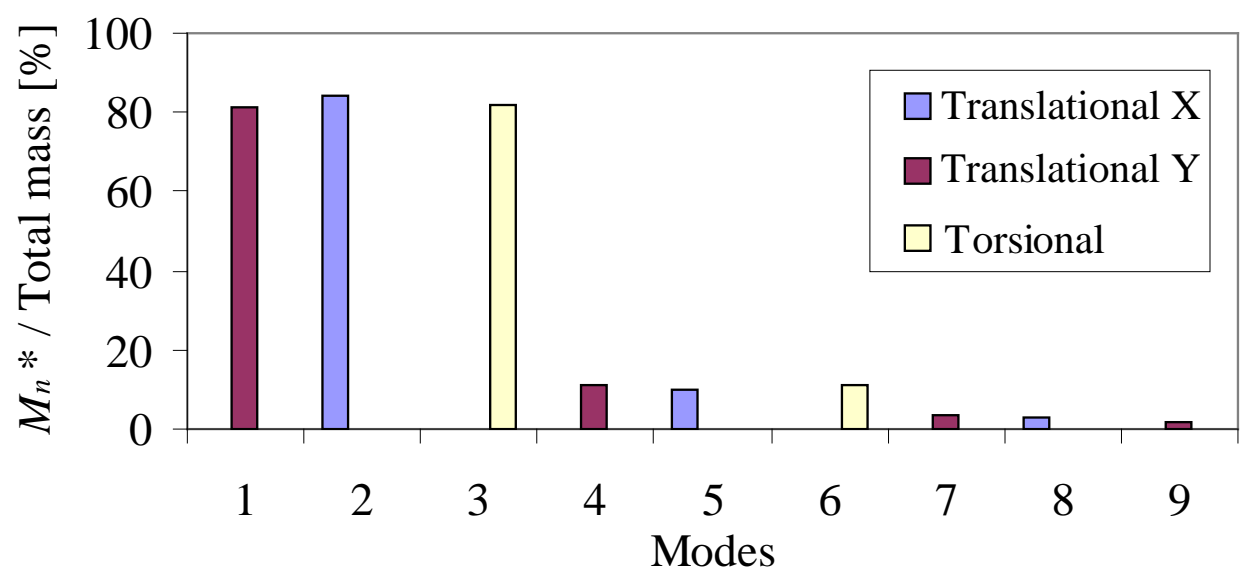

Figure 18. Effective modal masses, $M_{n}{ }^{*}$, for symmetric-plan building. \%, percent. 


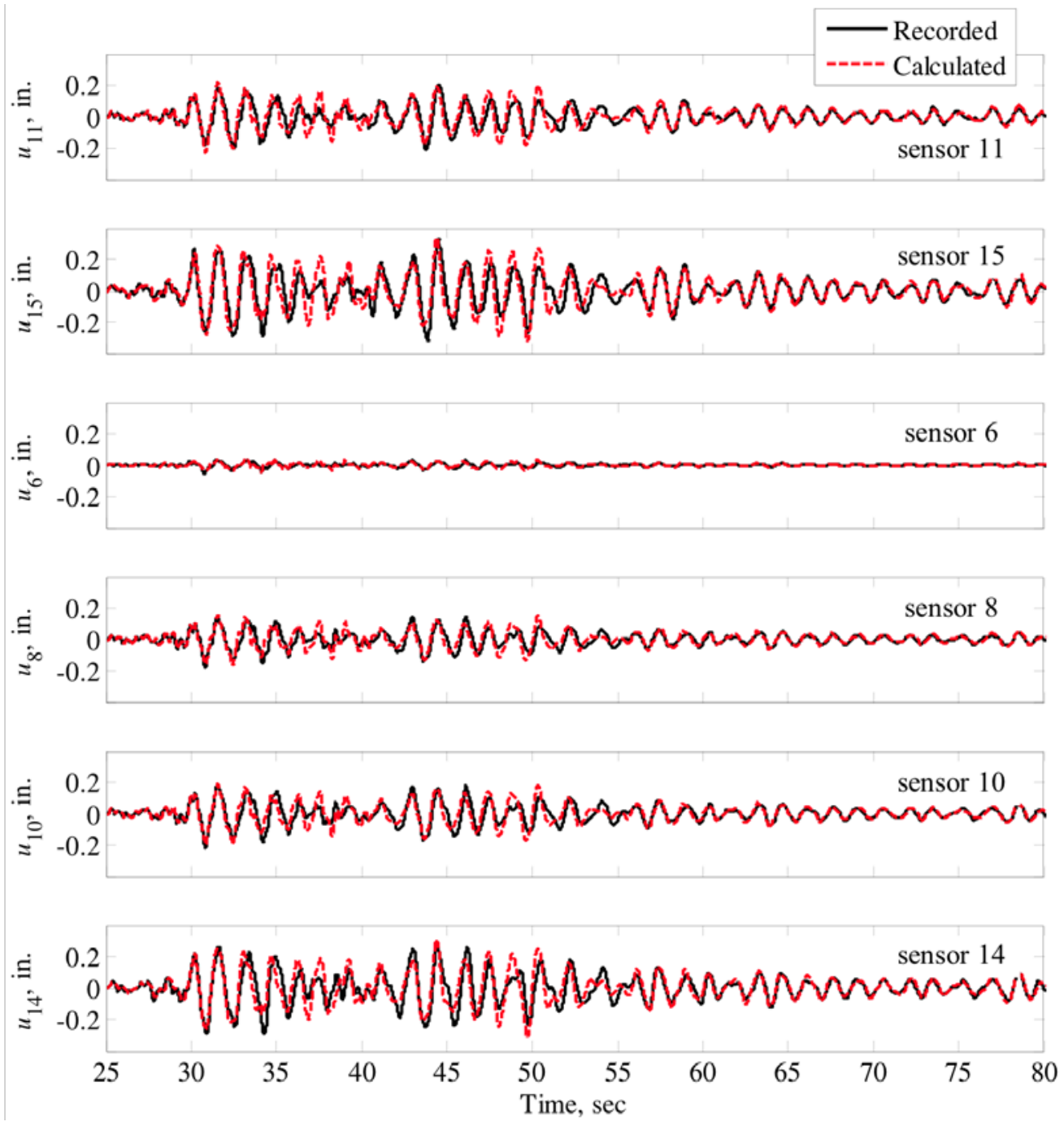

Figure 19. Comparison of recorded and computed floor displacements at the sensor locations (fig. 14) of symmetric-plan building. Recorded data is from the 2008 magnitude 5.4 Chino Hills southern California earthquake. 

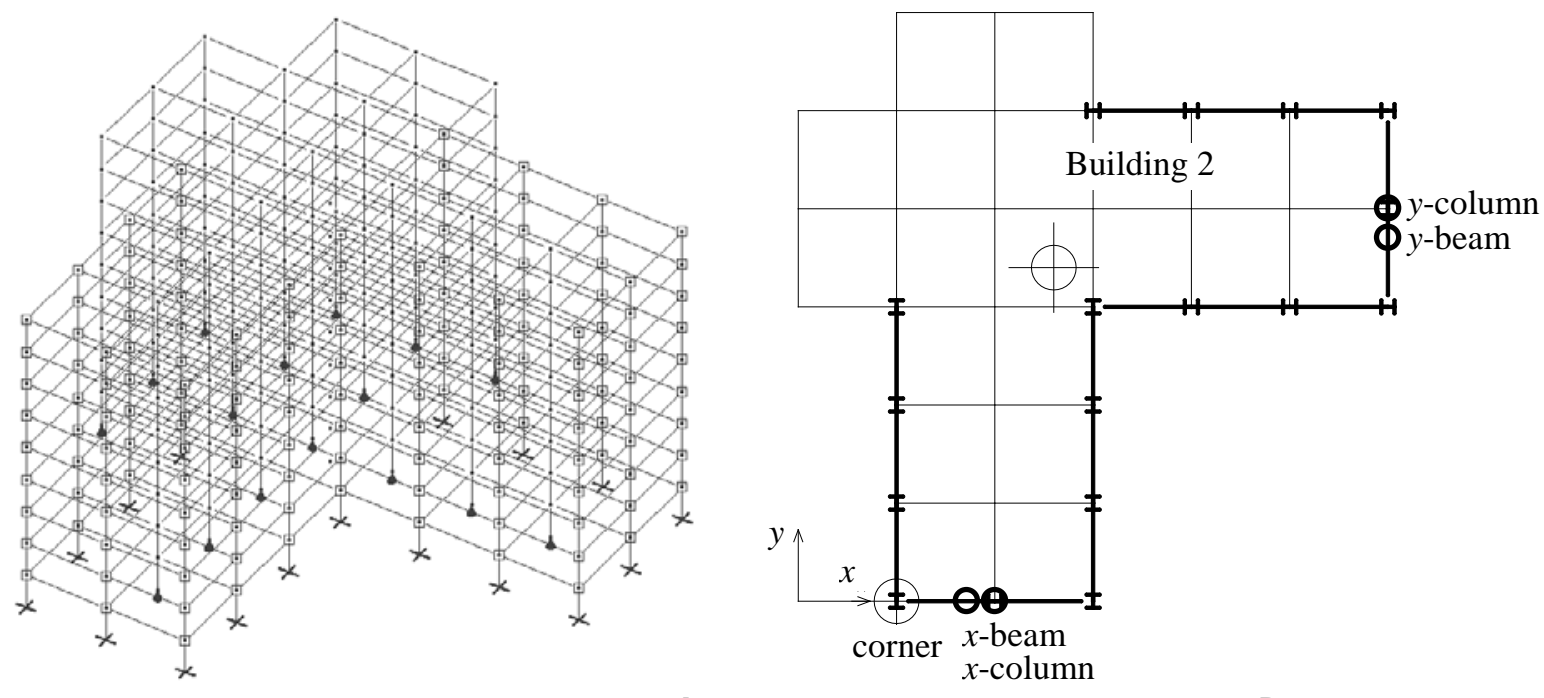

A

B

Figure 20. Nine-story asymmetric-plan building. $A$, three-dimensional computer model. $B$, plan view.

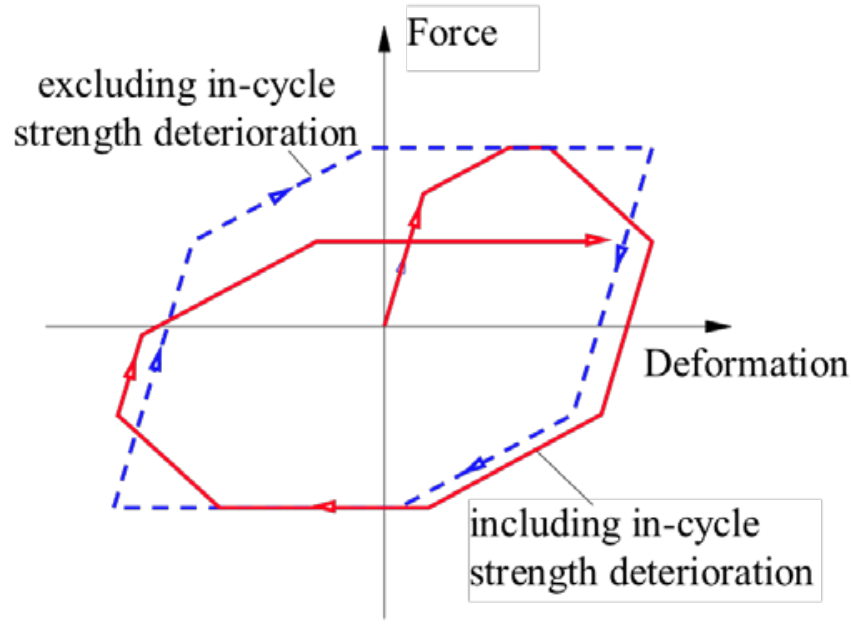

Figure 21. Trilinear force-deformation relations considering in-cycle strength deterioration (strength loss). 


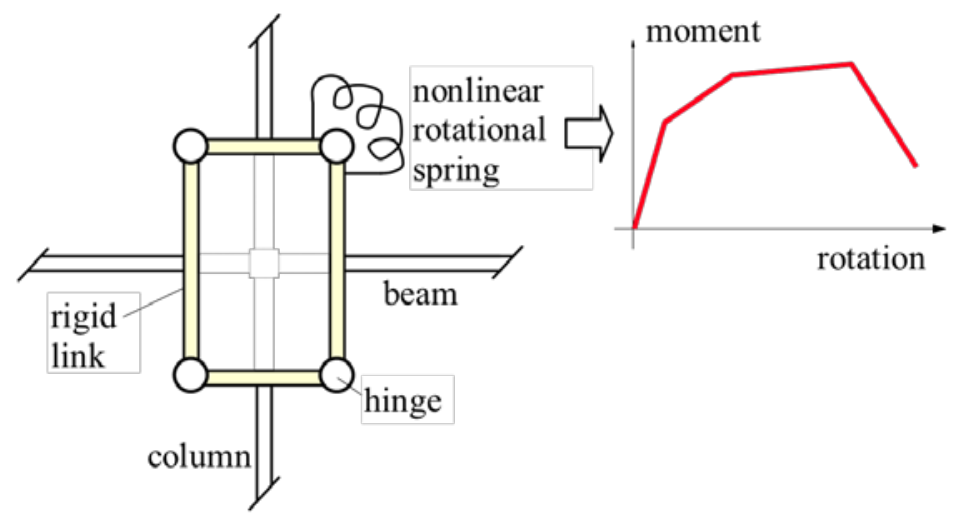

Figure 22. Krawinkler model for panel zones adapted from Computers and Structures, Inc. (2006).
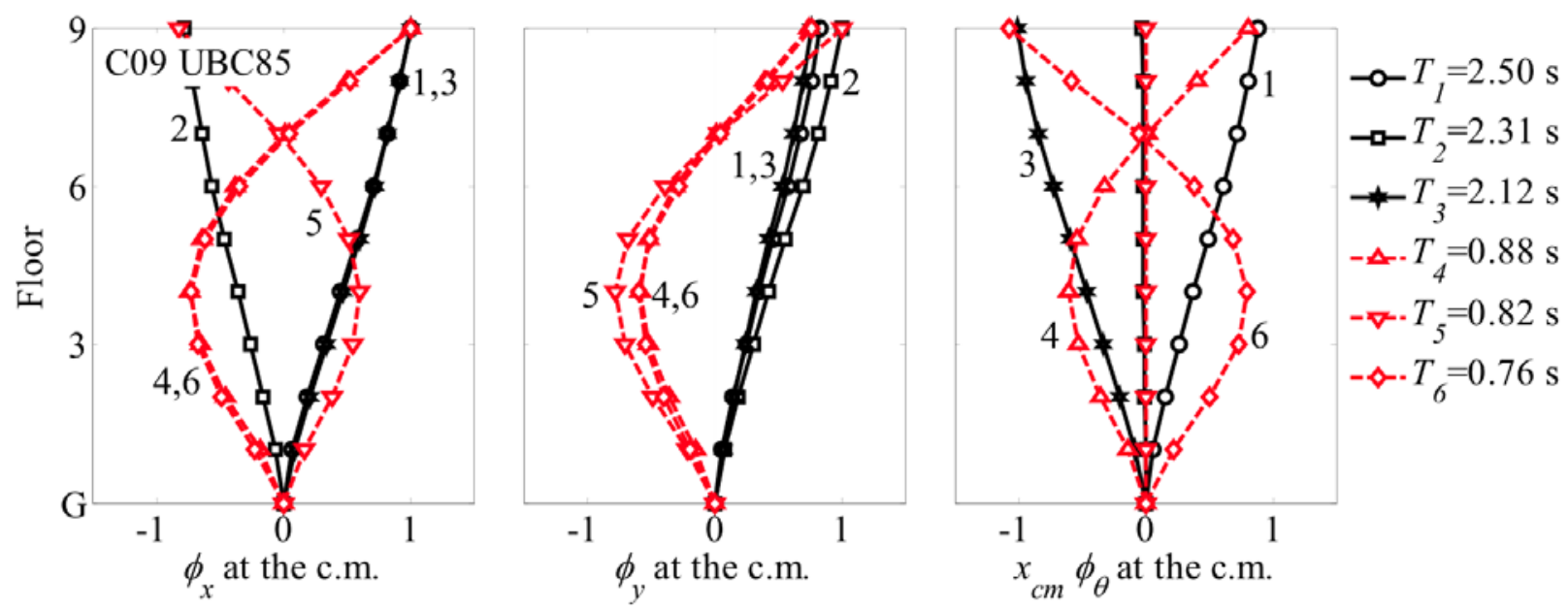

Figure 23. First six natural periods and modes of vibration of asymmetric-plan building; $x$-lateral, $y$-lateral and torsional motions are shown in the three columns. 
Mode 1

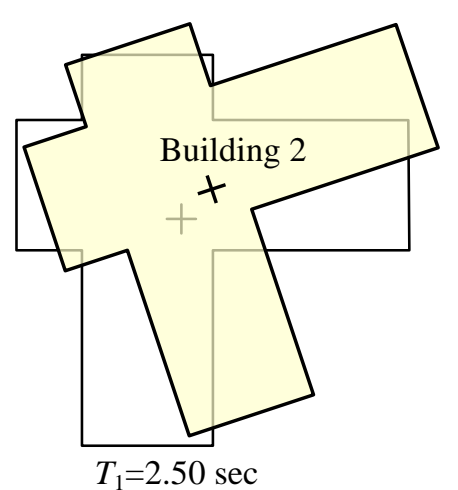

Mode 2

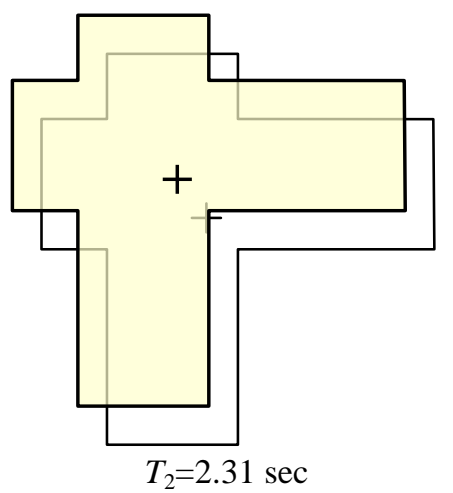

Mode 3

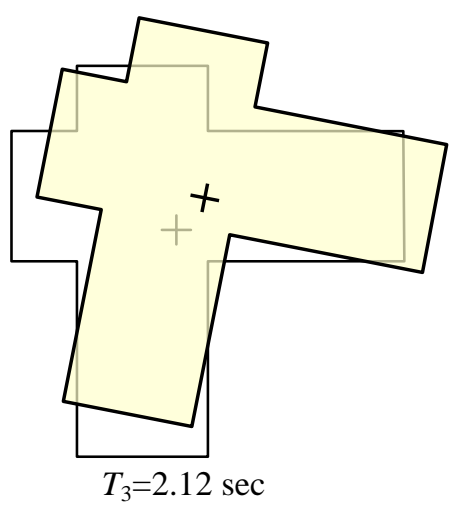

Figure 24. First triplet of periods and modes of vibration of asymmetric-plan building (only roof motion is shown), ( $T_{n}=$ elastic vibration period for the $n_{\text {th }}$ mode).

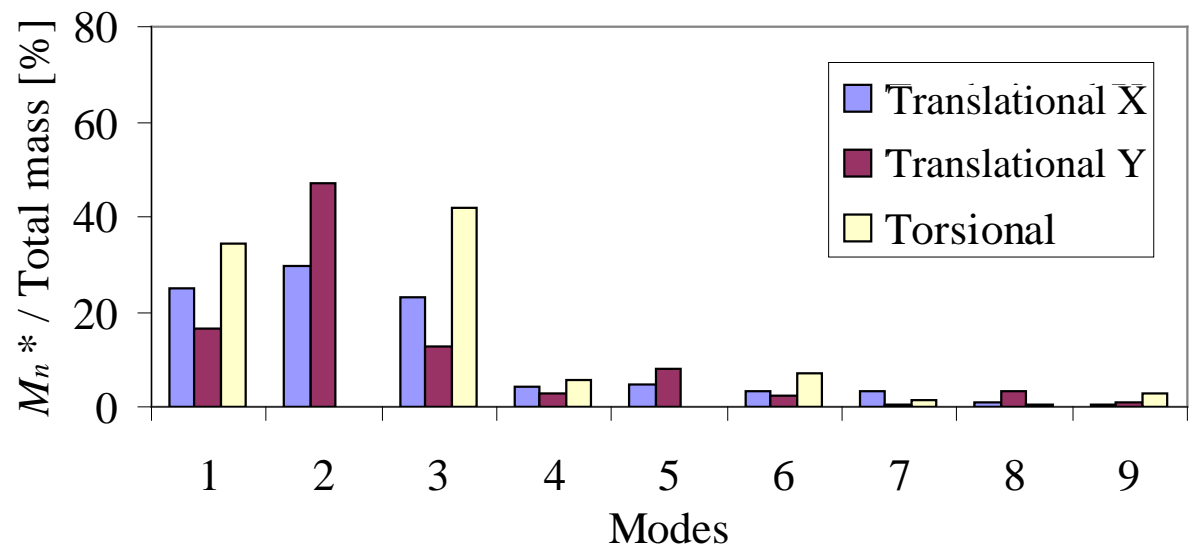

Figure 25. Effective modal masses, $M_{n}{ }^{*}$, for asymmetric-plan building. \%, percent. 

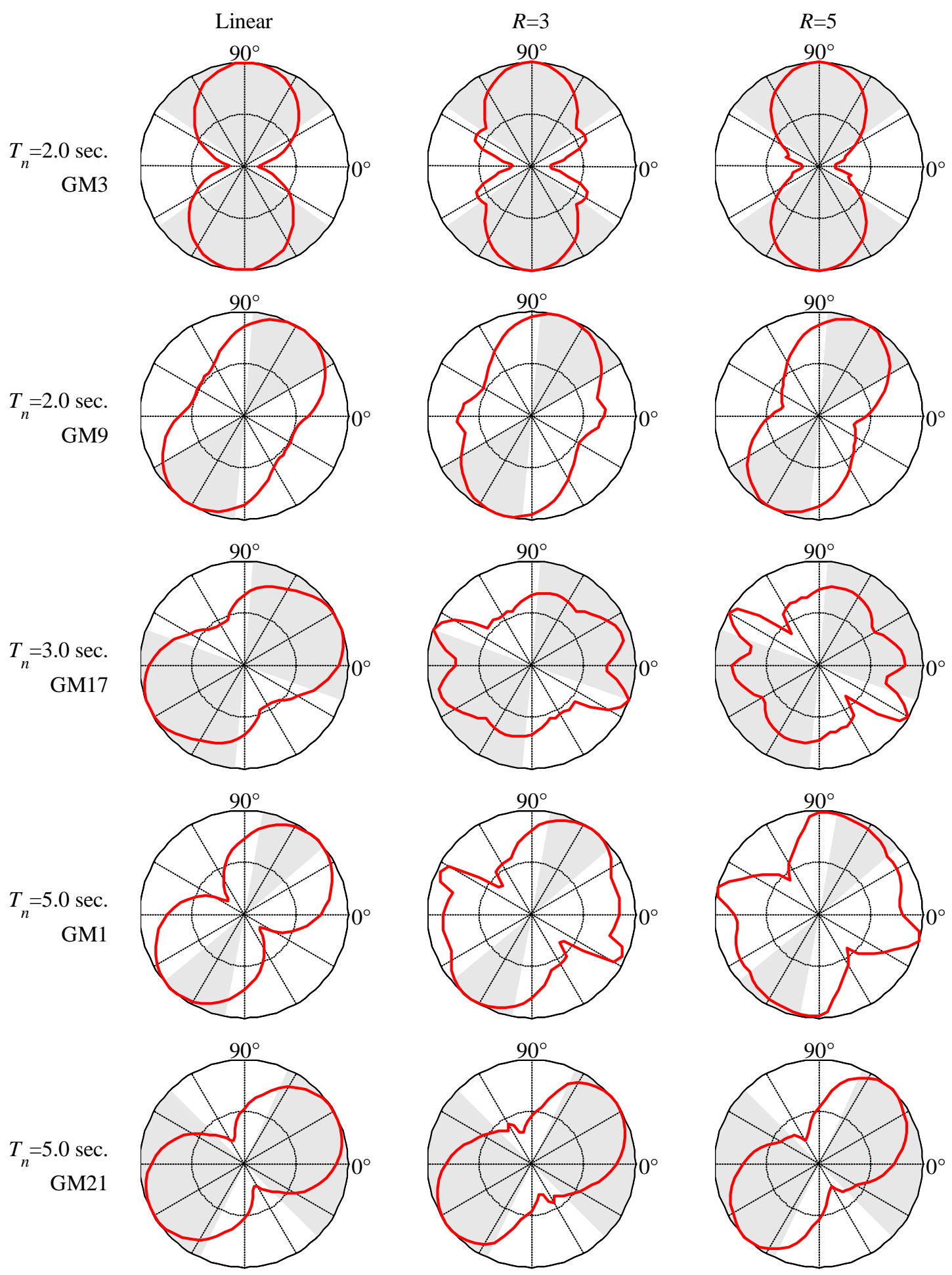

Figure 26. Displacement, $u_{x}$, at the center of mass (red line) as a function of the rotation angle, $\theta_{x}$, for single-story symmetric-plan systems with elastic first-mode vibration period $\left(T_{n}\right)=2,3$, and 5 seconds subjected to ground motions $(G M)$ with velocity pulse period close to $T_{n}$. The filled gray area shows values of $\theta_{x}$ in which velocity pulses are identified. Angles $\theta_{x}=0^{\circ}$ and $90^{\circ}$ correspond to the faultparallel and fault-normal directions, respectively. 

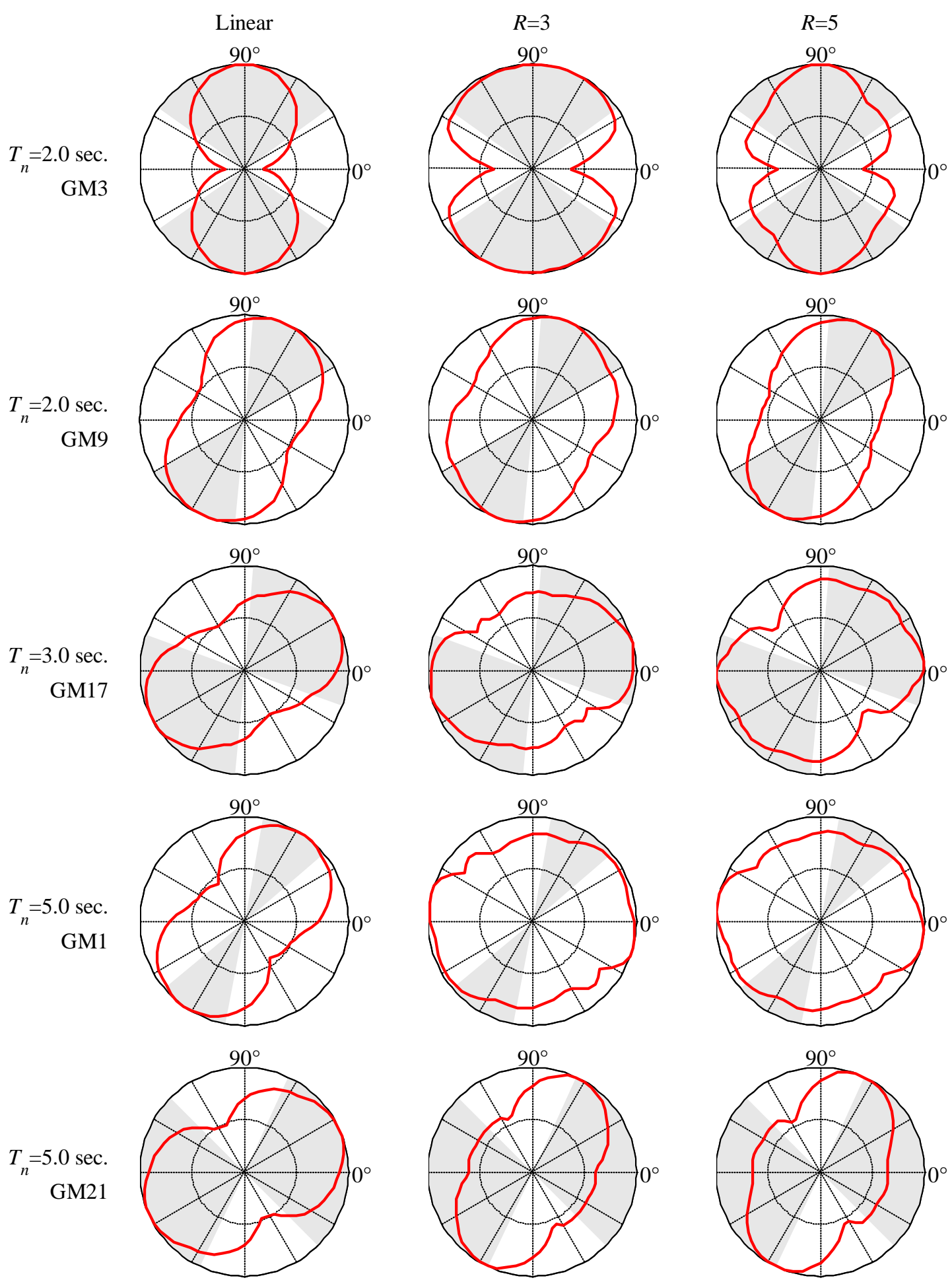

Figure 27. Floor total accelerations, $\ddot{u}_{x}$, at the center of mass (red line) as a function of the rotation angle, $\theta_{x}$, for single-story symmetric-plan systems with elastic first-mode vibration period $\left(T_{n}\right)=2,3$, and 5 seconds subjected to ground motions (GM) with velocity pulse period close to $T_{n}$. The filled gray area shows values of $\theta_{x}$ in which velocity pulses are identified for each record. Angles $\theta_{x}=0^{\circ}$ and $90^{\circ}$ correspond to the fault-parallel and fault-normal directions, respectively. 

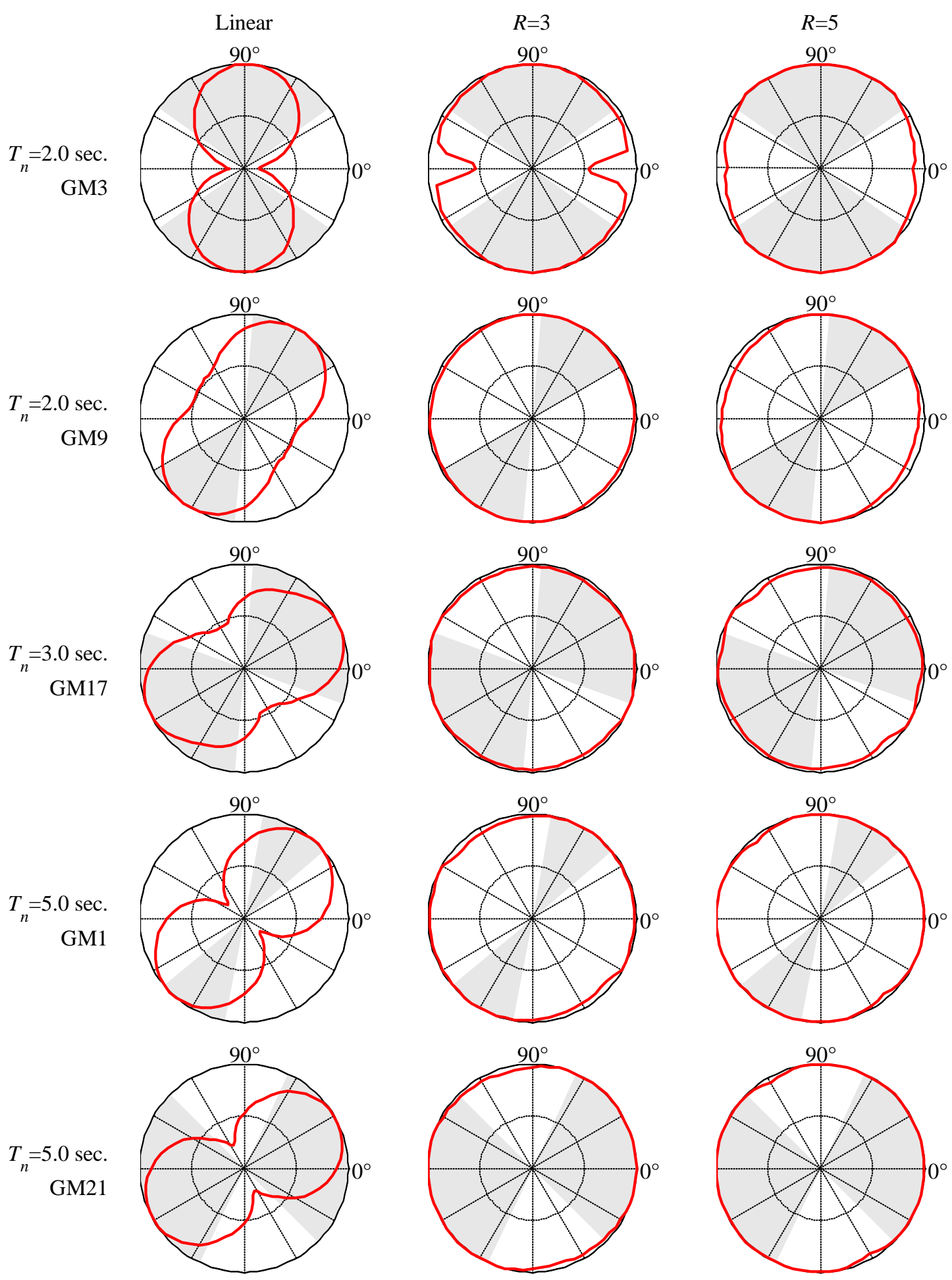

Figure 28. Force in bracing $b 1$ (red line) as a function of the rotation angle, $\theta_{x}$, for single-story symmetricplan systems with elastic first-mode vibration period $\left(T_{n}\right)=2,3$, and 5 seconds subjected to ground motions (GM) with velocity pulse period close to $T_{n}$. The filled gray area shows values of $\theta_{x}$ in which velocity pulses are identified for each record. Angles $\theta_{x}=0^{\circ}$ and $90^{\circ}$ correspond to the fault-parallel and fault-normal directions, respectively. 

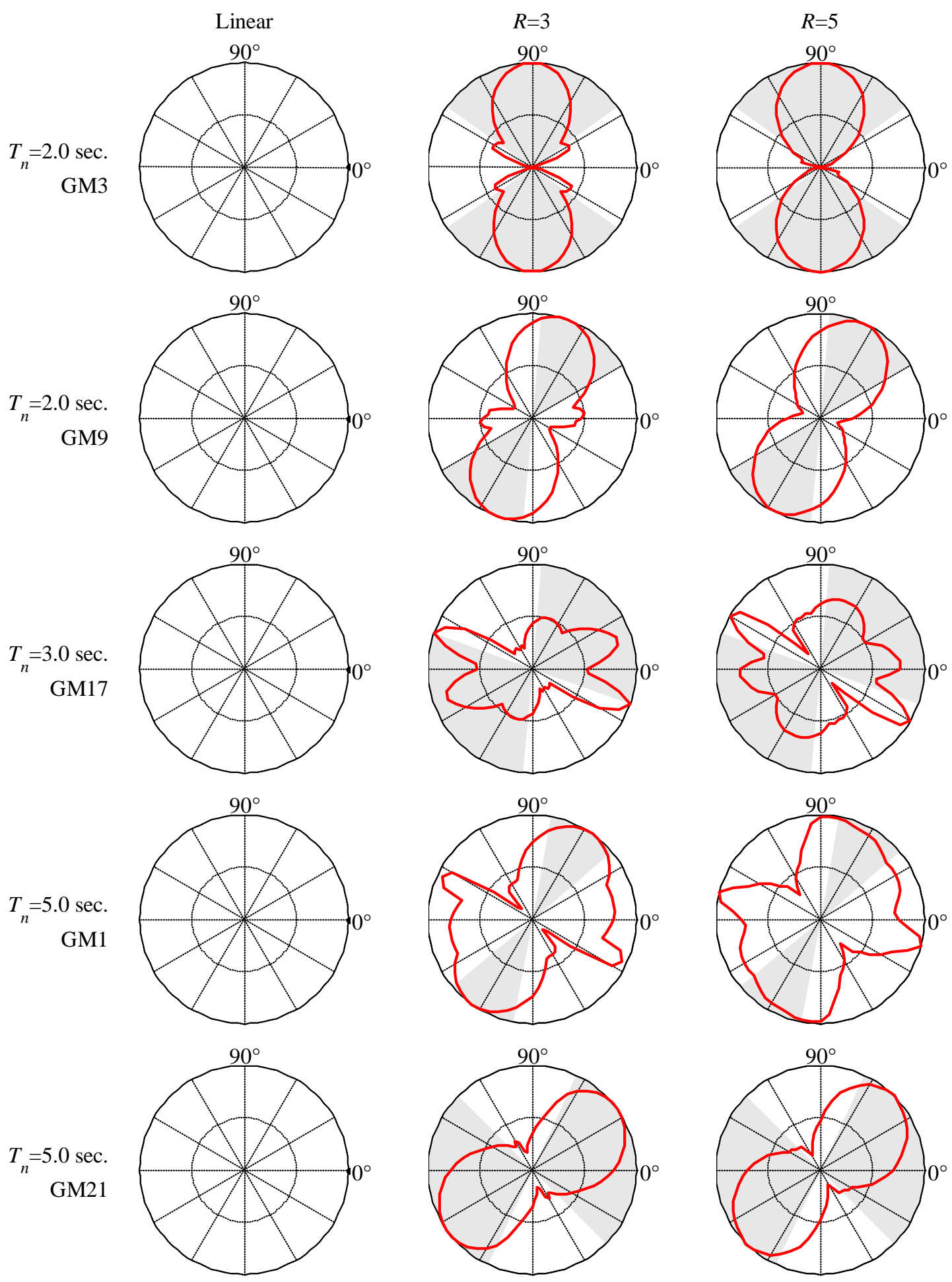

Figure 29. Plastic deformation in bracing $b 1$ (red line) as a function of the rotation angle, $\theta_{x}$, for singlestory symmetric-plan systems with elastic first-mode vibration period $\left(T_{n}\right)=2,3$, and 5 seconds subjected to ground motions $(G M)$ with velocity pulse period close to $T_{n}$. The filled gray area shows values of $\theta_{x}$ in which velocity pulses are identified for each record. Angles $\theta_{x}=0^{\circ}$ and $90^{\circ}$ correspond to the fault-parallel and fault-normal directions, respectively. 

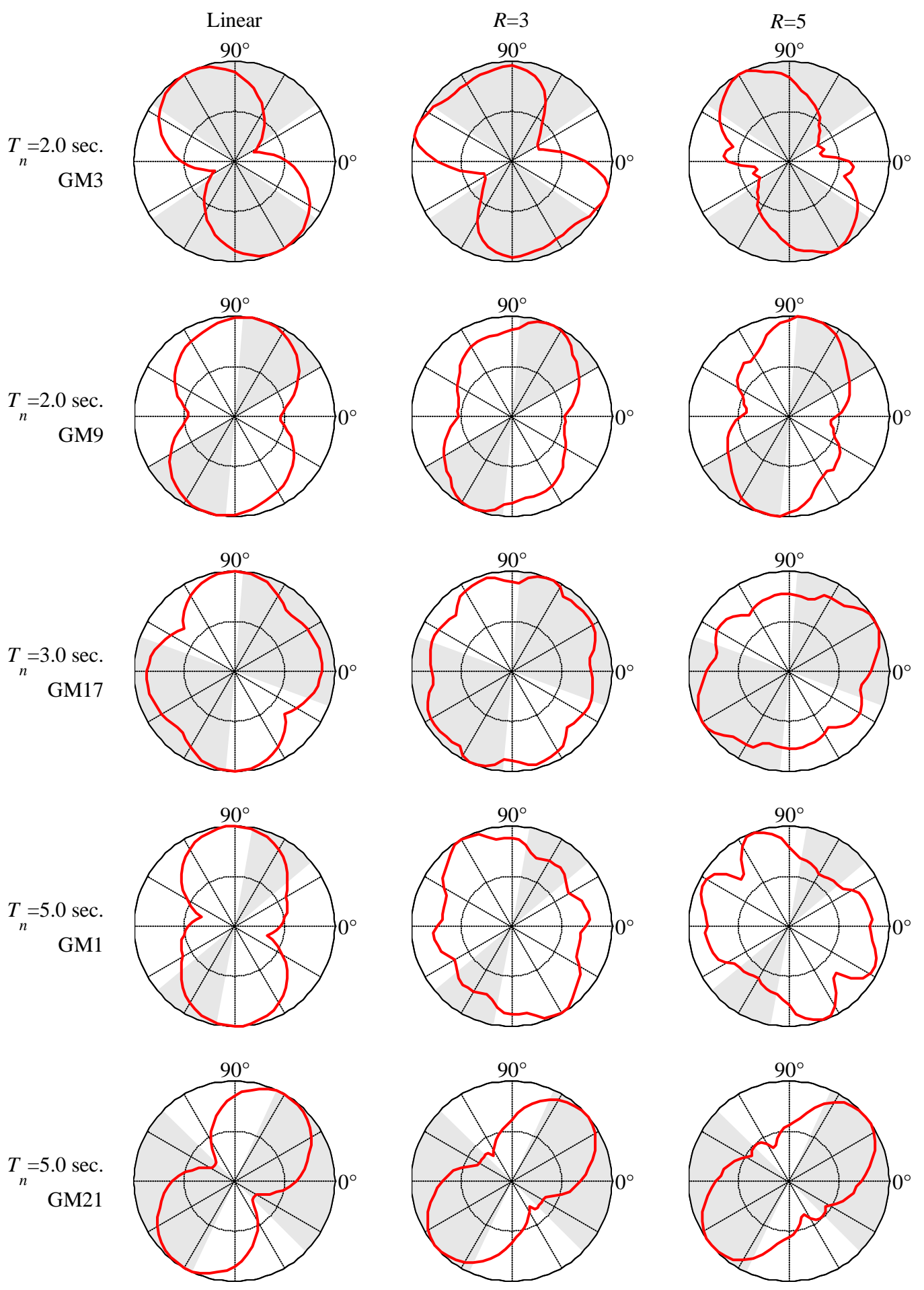

Figure 30. Displacement, $u_{x}$, at the center of mass (red line) as a function of the rotation angle, $\theta_{x}$, for single-story asymmetric-plan systems with elastic first-mode vibration period $\left(T_{n}\right)=2,3$, and 5 seconds subjected to ground motions (GM) with velocity pulse period close to $T_{n}$. The filled gray area shows values of $\theta_{x}$ in which velocity pulses are identified for each record. Angles $\theta_{x}=0^{\circ}$ and $90^{\circ}$ correspond to the fault-parallel and fault-normal directions, respectively. 

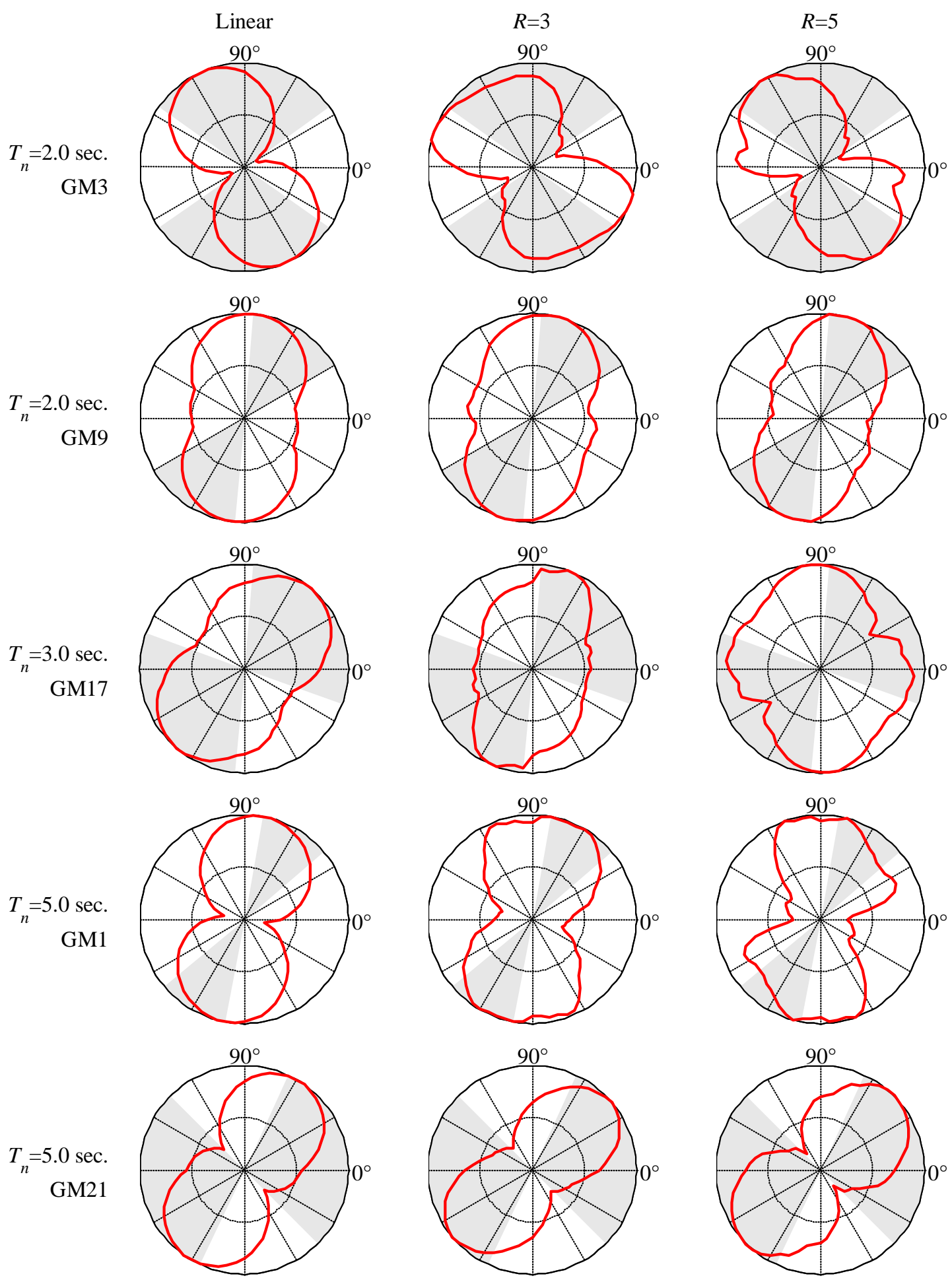

Figure 31. Displacement, $u_{x}$, at corner $p 2$ (red line) as a function of the rotation angle, $\theta_{x}$, for single-story asymmetric-plan systems with elastic first-mode vibration period $\left(T_{n}\right)=2,3$, and 5 seconds subjected to ground motions (GM) with velocity pulse period close to $T_{n}$. The filled gray area shows values of $\theta_{x}$ in which velocity pulses are identified for each record. Angles $\theta_{x}=0^{\circ}$ and $90^{\circ}$ correspond to the faultparallel and fault-normal directions, respectively. 

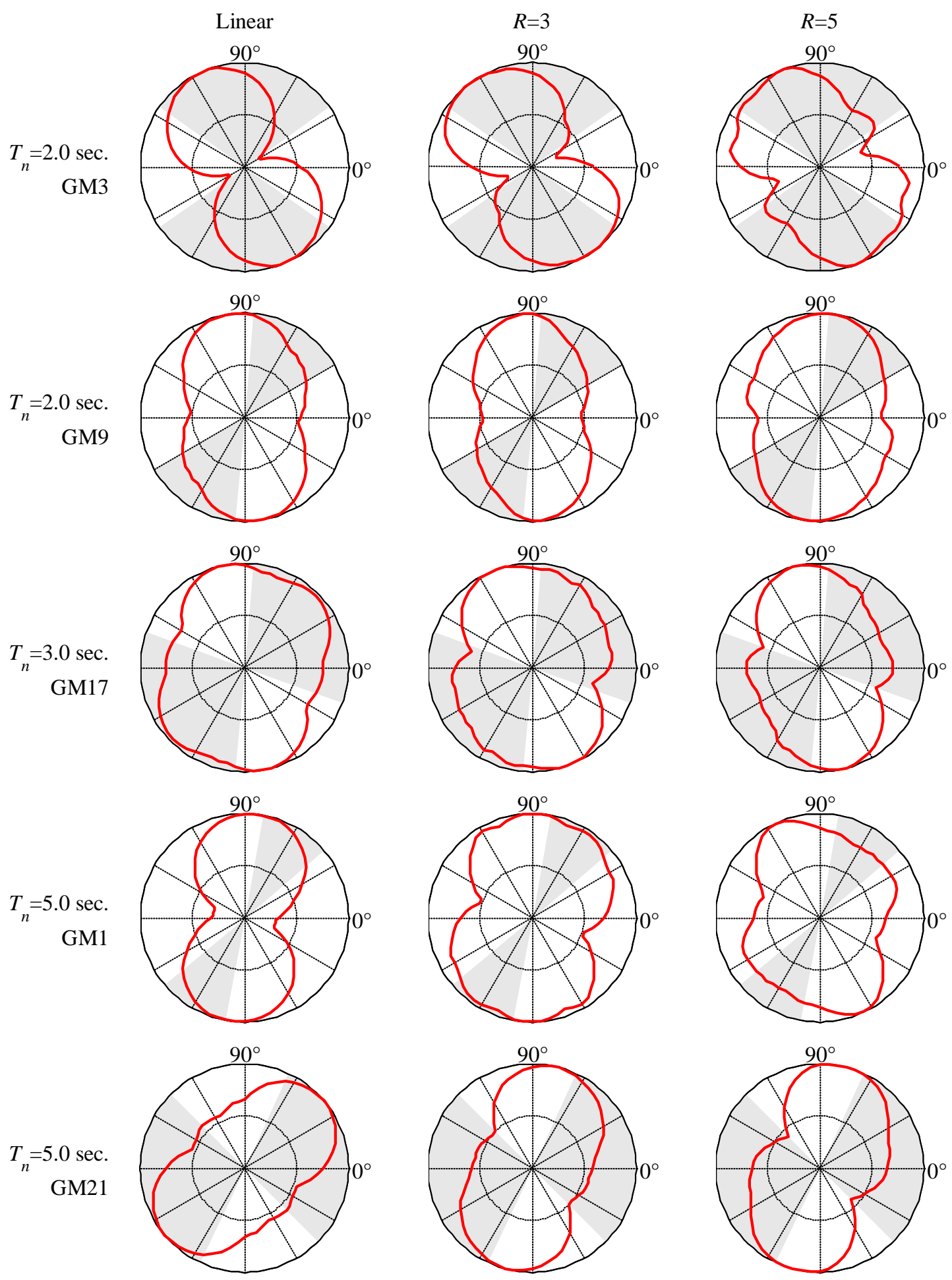

Figure 32. Floor total accelerations, $\ddot{u}_{x}$, at corner $\mathrm{p} 2$ (red line) as a function of the rotation angle, $\theta_{x}$, for asymmetric-plan buildings with elastic first-mode vibration period $\left(T_{n}\right)=2,3$, and 5 seconds subjected to ground motions (GM) with velocity pulse period close to $T_{n}$. The filled gray area shows values of $\theta_{x}$ in which velocity pulses are identified for each record. Angles $\theta_{x}=0^{\circ}$ and $90^{\circ}$ correspond to the faultparallel and fault-normal directions, respectively. 

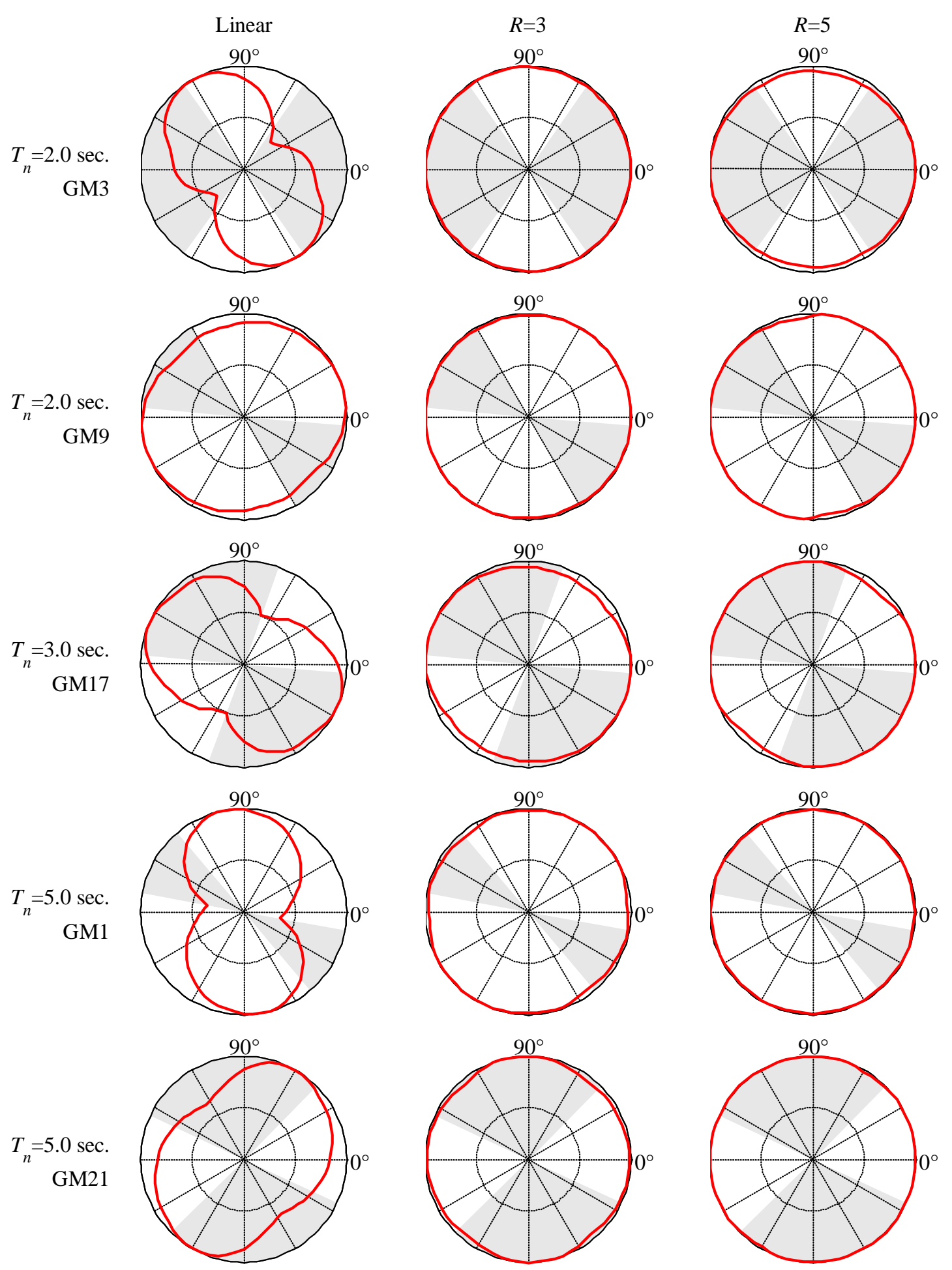

Figure 33. Force in bracing b3 (red line) as a function of the rotation angle, $\theta_{x}$, for single-story asymmetricplan systems with elastic first-mode vibration period $\left(T_{n}\right)=2,3$, and 5 seconds subjected to ground motions (GM) with velocity pulse period close to $T_{n}$. The filled gray area shows values of $\theta_{x}$ in which velocity pulses are identified for each record. Angles $\theta_{x}=0^{\circ}$ and $90^{\circ}$ correspond to the fault-parallel and fault-normal directions, respectively. 

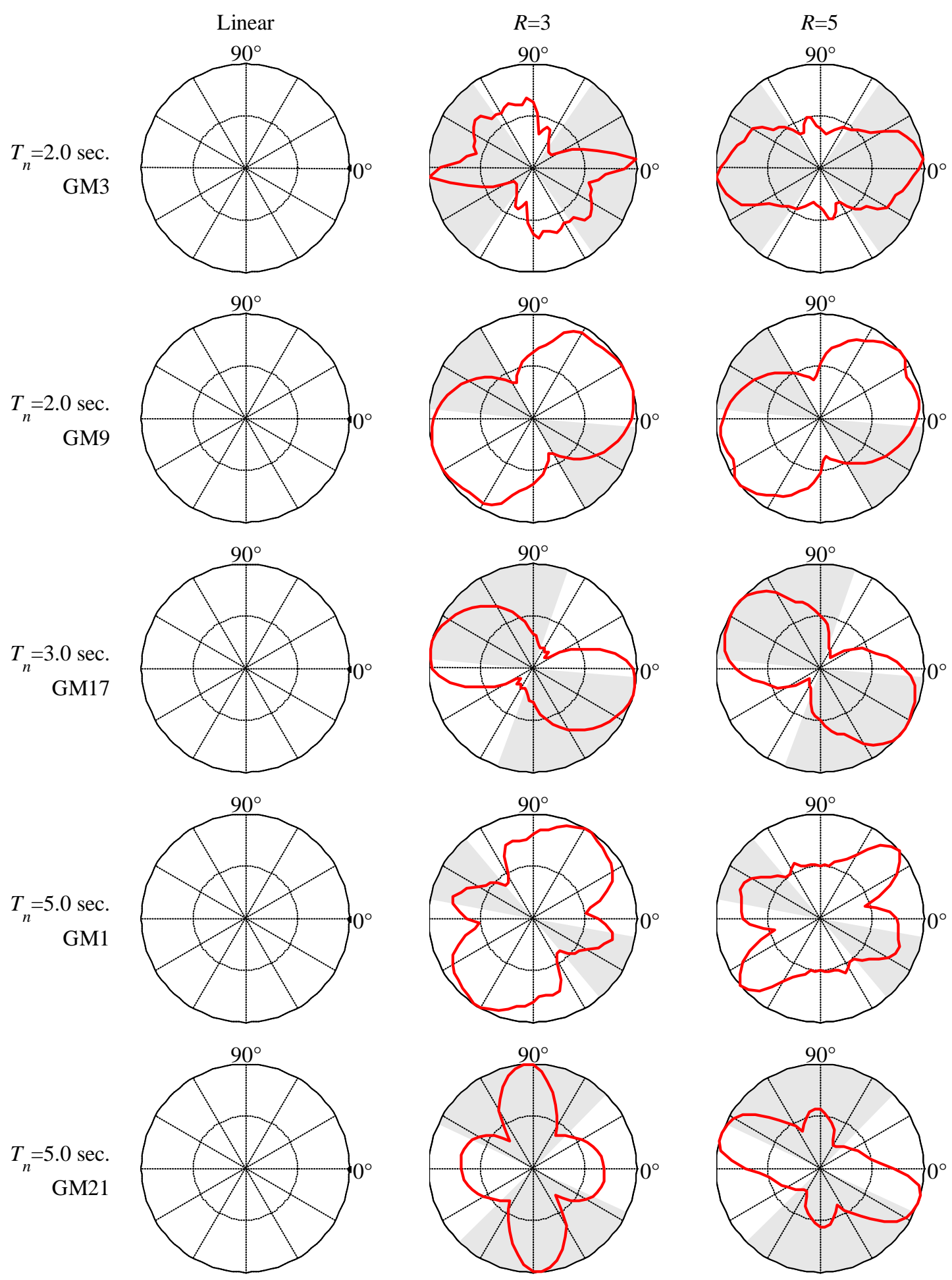

Figure 34. Plastic deformation in bracing b3 (red line) as a function of the rotation angle, $\theta_{x}$, for singlestory asymmetric-plan systems with elastic first-mode vibration period $\left(T_{n}\right)=2,3$, and 5 seconds subjected to ground motions $(\mathrm{GM})$ with velocity pulse period close to $T_{n}$. The filled gray area shows values of $\theta_{x}$ in which velocity pulses are identified for each record. Angles $\theta_{x}=0^{\circ}$ and $90^{\circ}$ correspond to the fault-parallel and fault-normal directions, respectively. 

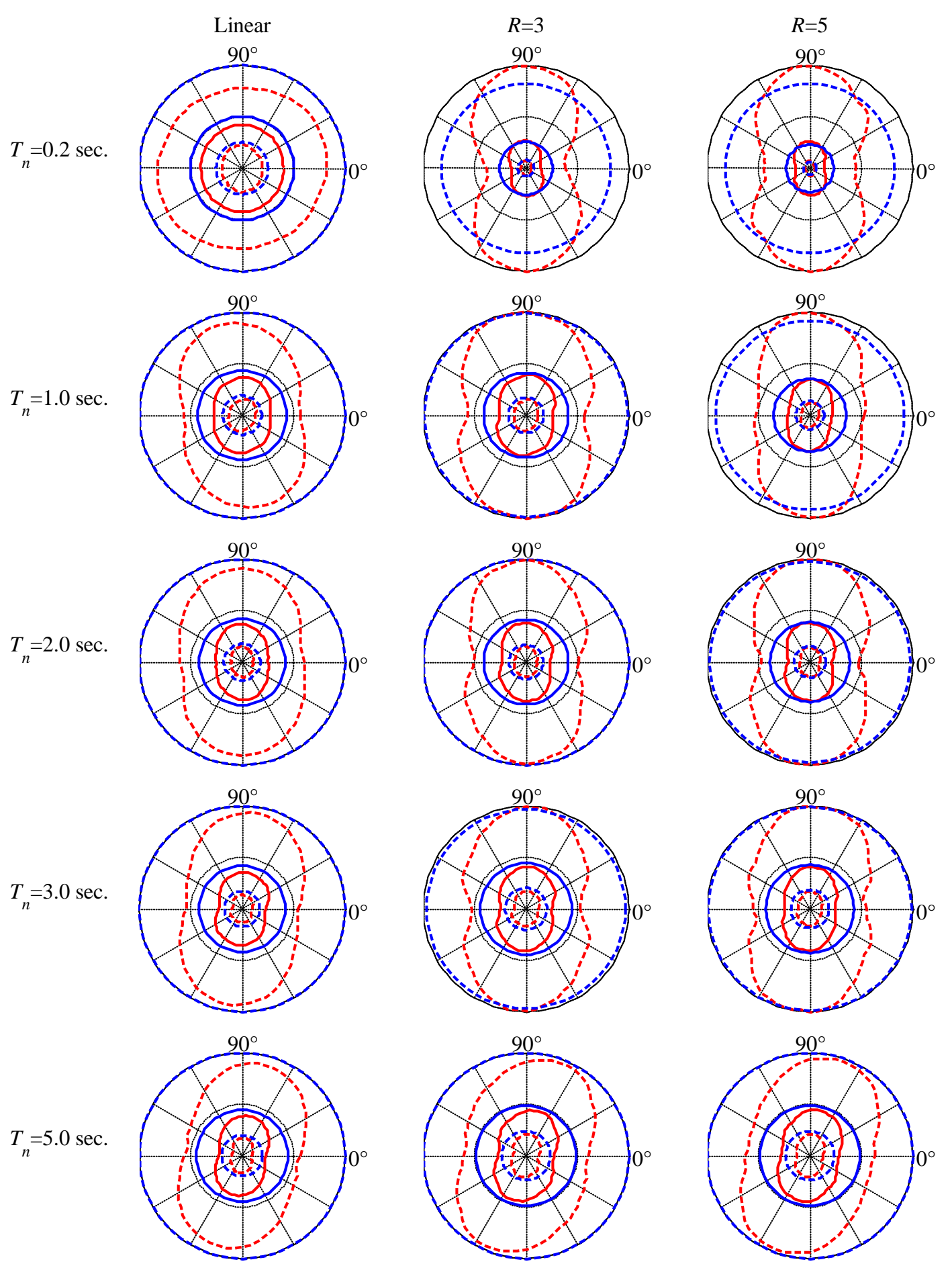

Median displacement + /- one standard deviation due to components rotated $\theta_{x}$ degrees Median displacement +/- one standard deviation due to MD components

Figure 35. Median displacements, $u_{x}$, at the center of mass as a function of the rotation angle, $\theta_{x}$, for single-story symmetric-plan systems with elastic first-mode vibration period $\left(T_{n}\right)=0.2,1,2,3$, and 5 seconds subjected to bidirectional loading. The red lines represent the median displacement $u_{x} \pm \sigma$. The blue circles represent the median displacement, $u_{x m} \pm \sigma$, for the systems subjected to bidirectional 

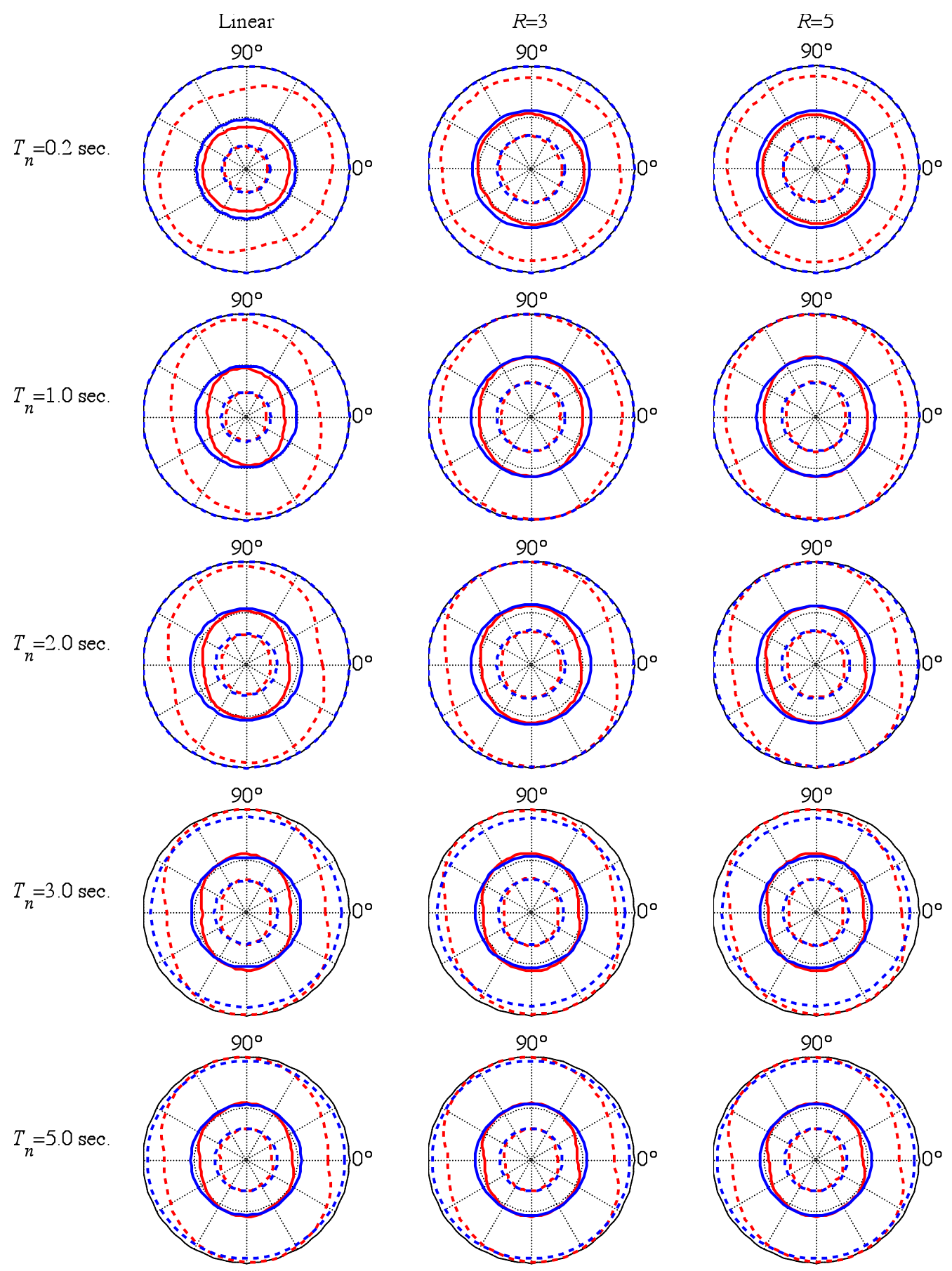

I:-1.:- Median acceleration $+/$ - one standard deviation due to components rotated $\theta_{x}$ degrees -1:-: Median acceleration $+/$ - one standard deviation due to MD components

Figure 36. Median total-accelerations, $\ddot{u}_{x}{ }_{x}$, at the center of mass as a function of the rotation angle, $\theta_{x}$, for single-story symmetric-plan systems with elastic first-mode vibration period $\left(T_{n}\right)=0.2,1,2,3$, and 5 seconds subjected to bidirectional loading. The red lines represent the median displacement, $\ddot{u}_{x} \pm \sigma$. The blue circles represent the median total-acceleration, $\ddot{u}_{x m} \pm \sigma$, for the systems subjected to bidirectional ground motions in the maximum direction. Solid lines are 50th and dashed lines are16th and 84th percentile engineering demand parameters (EDPs). MD, maximum direction. 

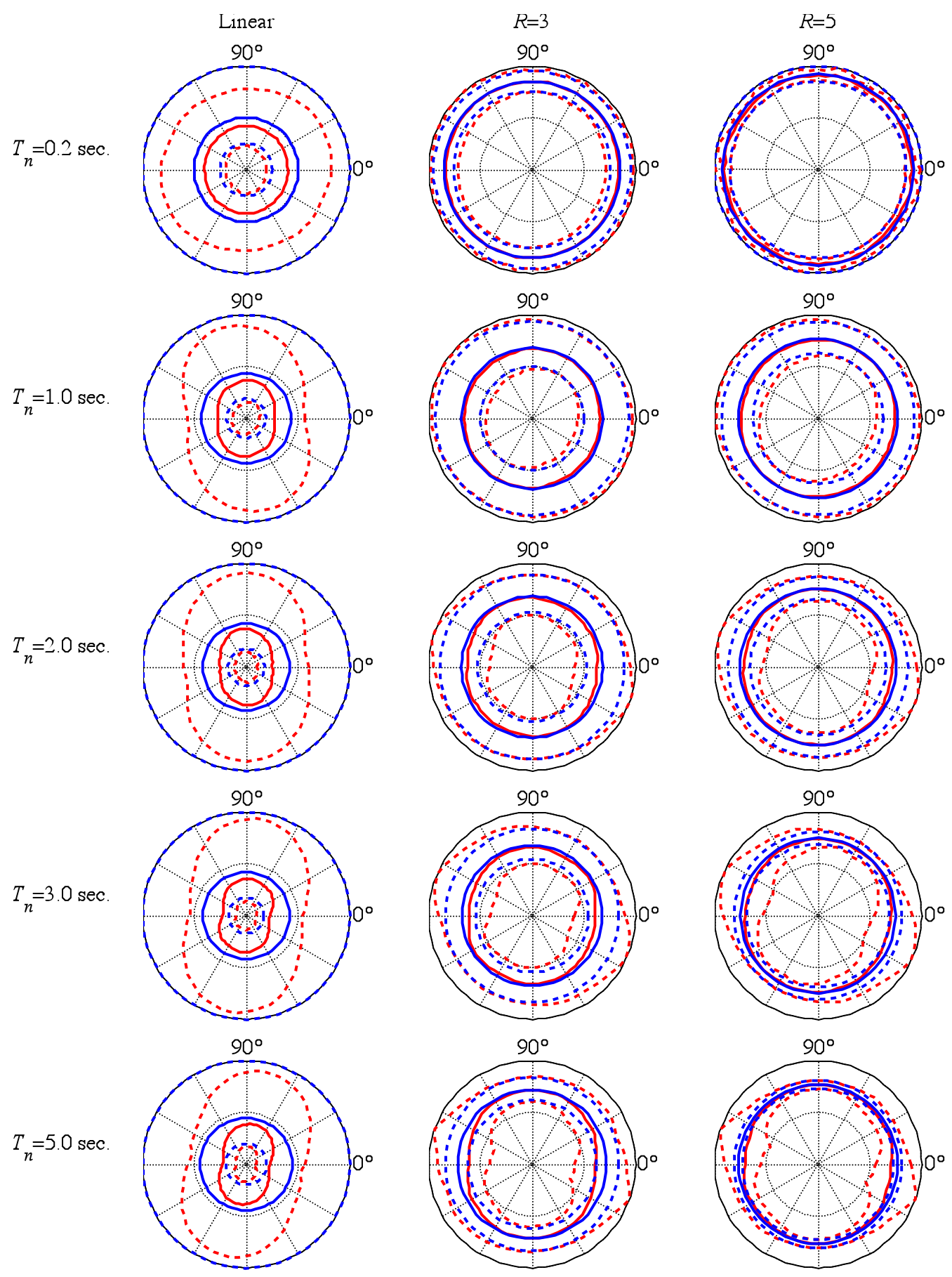

.-.-.: Median brace force $+/$ - one standard deviation due to components rotated $\theta_{x}$ degrees Median brace force $+/$ - one standard deviation due to MD components

Figure 37. Median force $q_{1}$ in bracing b1 as a function of the rotation angle, $\theta_{x}$, for single-story symmetricplan systems elastic first-mode vibration period $\left(T_{n}\right)=0.2,1,2,3$, and 5 seconds subjected to bidirectional loading. The red lines represent the median force, $q_{1} \pm \sigma$. The blue circles represent the median force, $q_{1} \pm \sigma$, for the systems subjected to bidirectional ground motions in the maximum direction. Solid lines are 50th and dashed lines are16th and 84th percentile engineering demand parameters (EDPs). MD, maximum direction. 

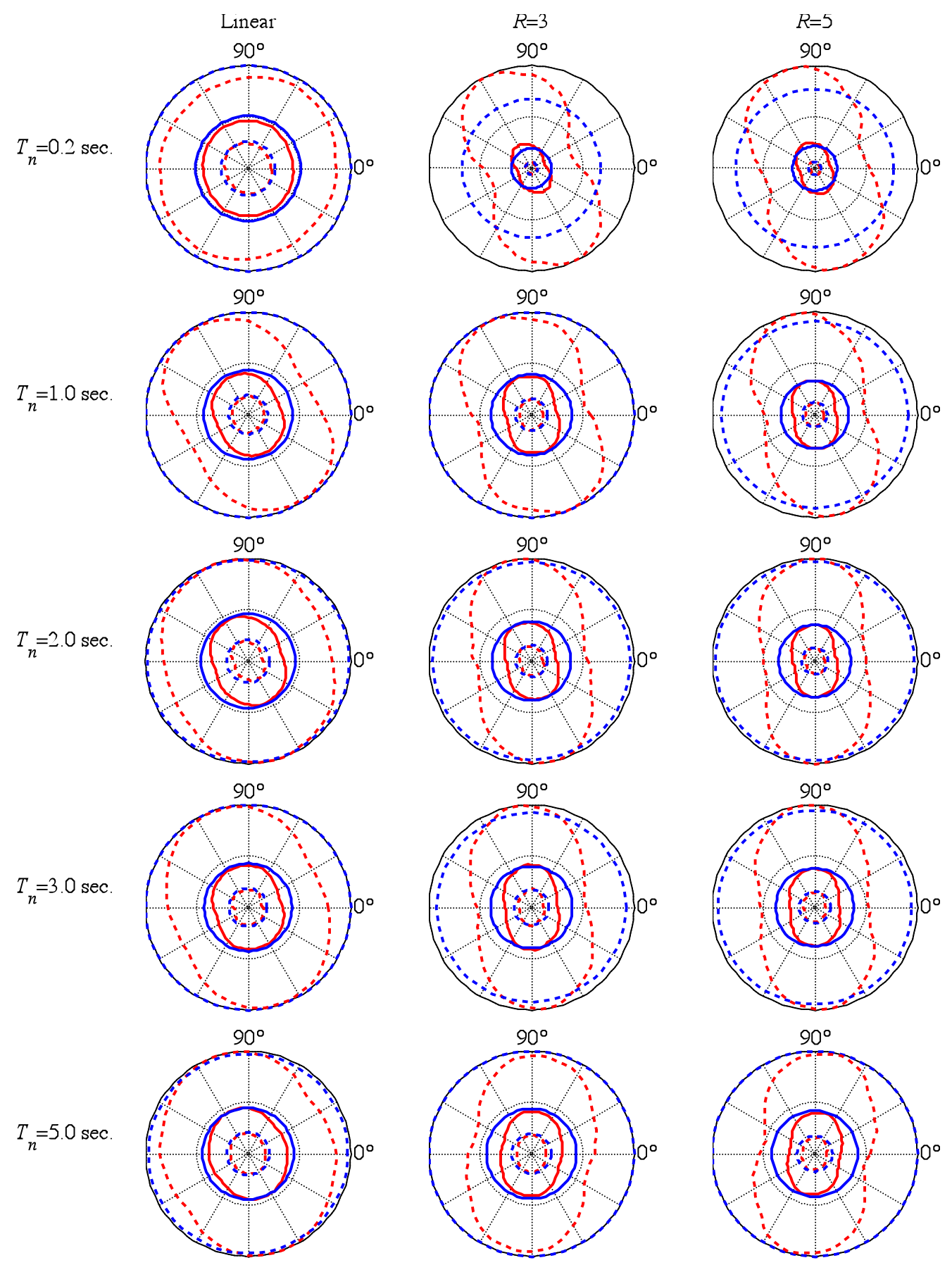

Median displacement $+/$ - one standard deviation due to components rotated $\theta_{x}$ degrees :-1:- Median displacement $+/$ - one standard deviation due to MD components

Figure 38. Median displacements, $u_{x}$, at the center of mass as a function of the rotation angle, $\theta_{x}$, for single-story asymmetric-plan systems with elastic first-mode vibration period $\left(T_{n}\right)=0.2,1,2,3$, and 5 seconds subjected to bidirectional loading. The red lines represent the median displacement $u_{x} \pm \sigma$. The blue circles represent the median displacement $u_{x m} \pm \sigma$ for the systems subjected to bidirectional ground motions in the maximum direction. Solid lines are 50th and dashed lines are16th and 84th percentile engineering demand parameters (EDPs). MD, maximum direction. 

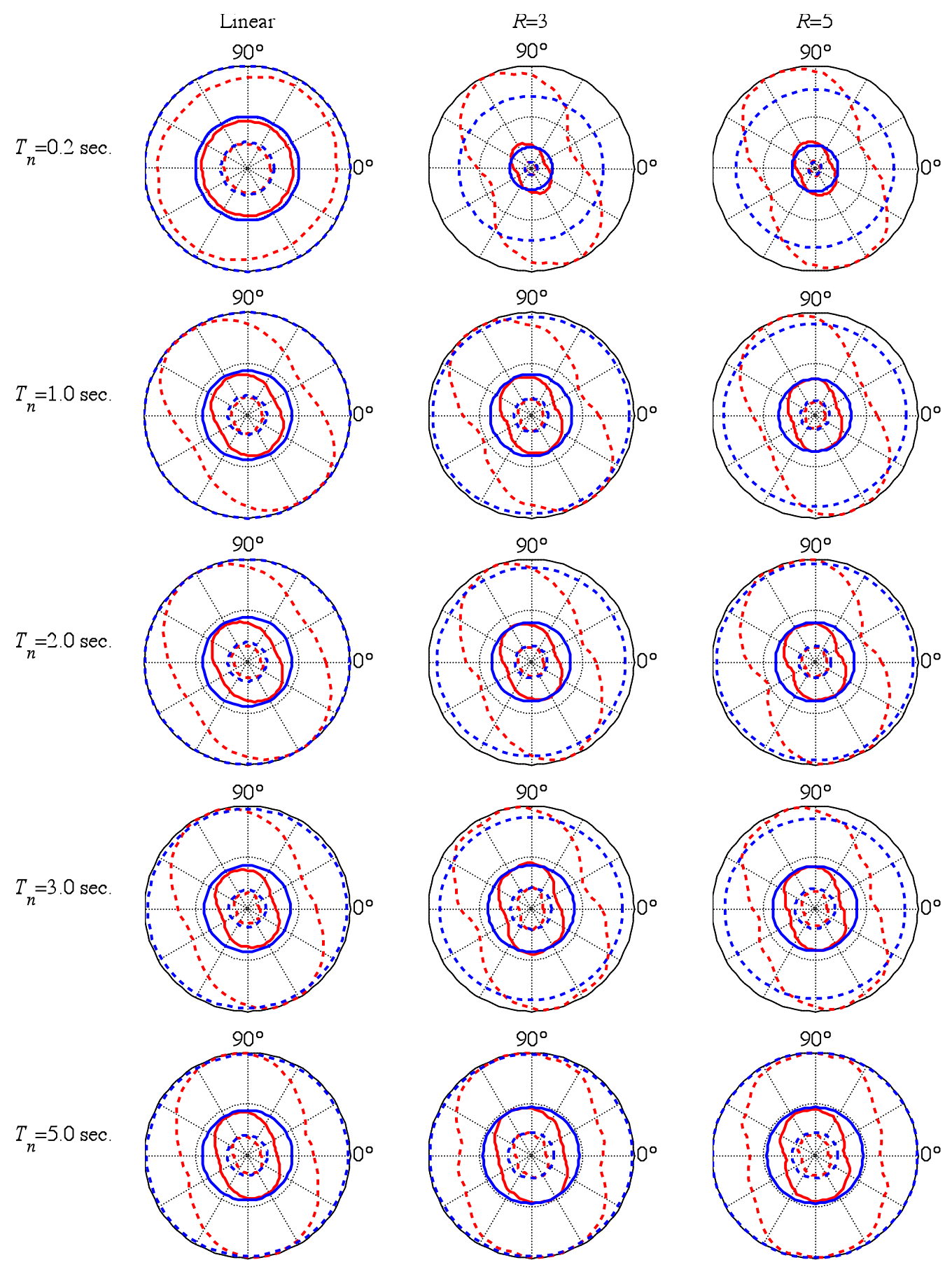

....-.: Median displacement $+/$ - one standard deviation due to components rotated $\theta_{x}$ degrees -...:- Median displacement +/- one standard deviation due to MD components

Figure 39. Median displacements, $u_{x}$, at corner $\mathrm{p} 2$ as a function of the rotation angle, $\theta_{x}$, for single-story asymmetric-plan systems with elastic first-mode vibration period $\left(T_{n}\right)=0.2,1,2,3$, and 5 seconds subjected to bidirectional loading. The red lines represent the median displacement, $u_{x} \pm \sigma$. The blue circles represent the median displacement, $u_{x m} \pm \sigma$, for the systems subjected to bidirectional ground motions in the maximum direction. Solid lines are 50th and dashed lines are16th and 84th percentile engineering demand parameters (EDPs). MD, maximum direction. 

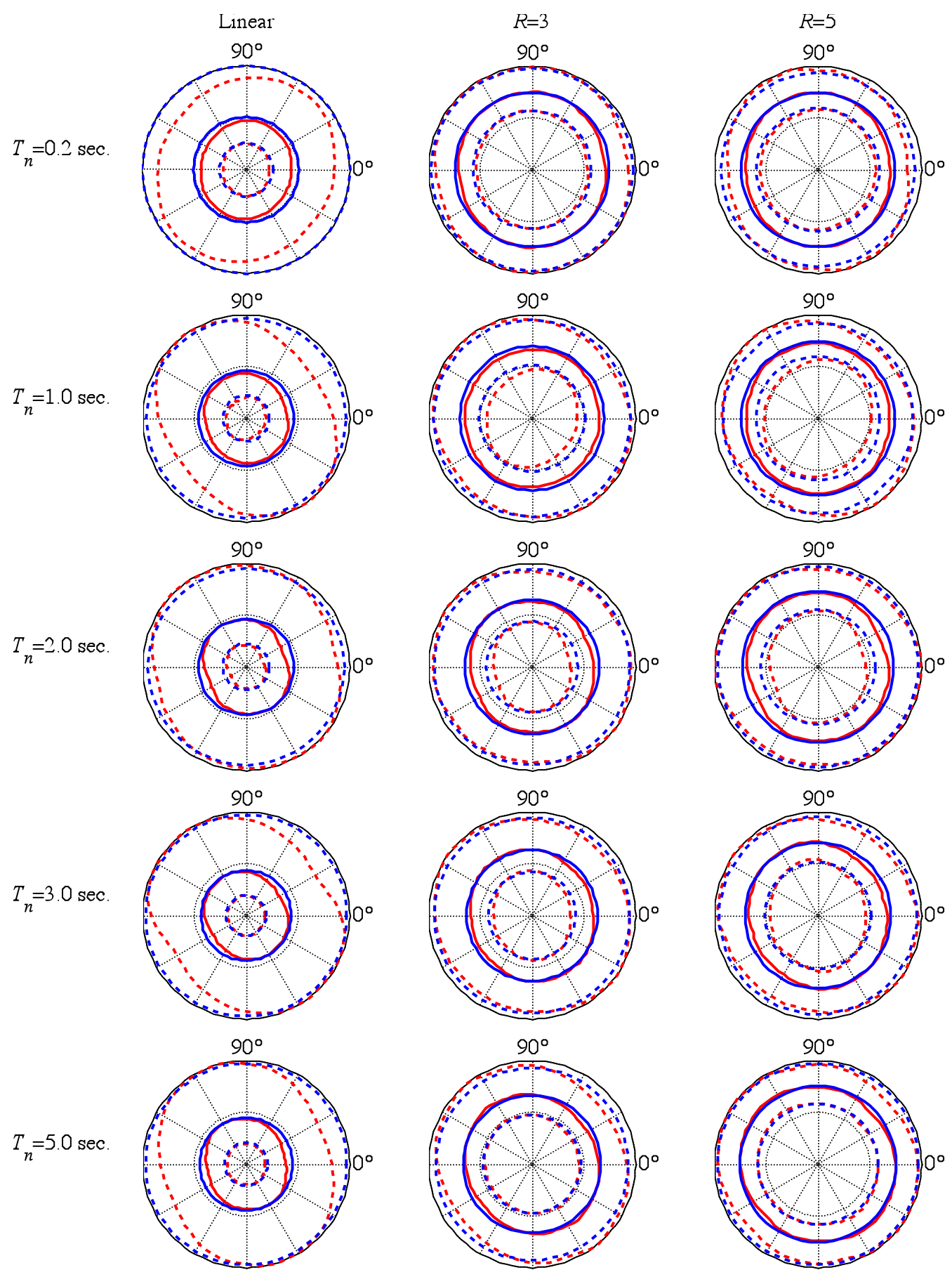

-..--: Median acceleration $+/$ - one standard deviation due to components rotated $\theta_{x}$ degrees Median acceleration +/- one standard deviation due to MD components

Figure 40. Median total-accelerations, $\ddot{u}_{x}^{t}$, at corner $\mathrm{p} 2$ as a function of the rotation angle, $\theta_{x}$, for singlestory asymmetric-plan systems with elastic first-mode vibration period $\left(T_{n}\right)=0.2,1,2,3$, and 5 seconds subjected to bidirectional loading. The red lines represent the median displacement, $\ddot{u}_{x} \pm \sigma$. The blue circles represent the median total-acceleration, $\ddot{u}_{\mathrm{x} m} \pm \sigma$, for the systems subjected to bidirectional ground motions in the maximum direction. Solid lines are 50th and dashed lines are16th and 84th percentile engineering demand parameters (EDPs). MD, maximum direction. 

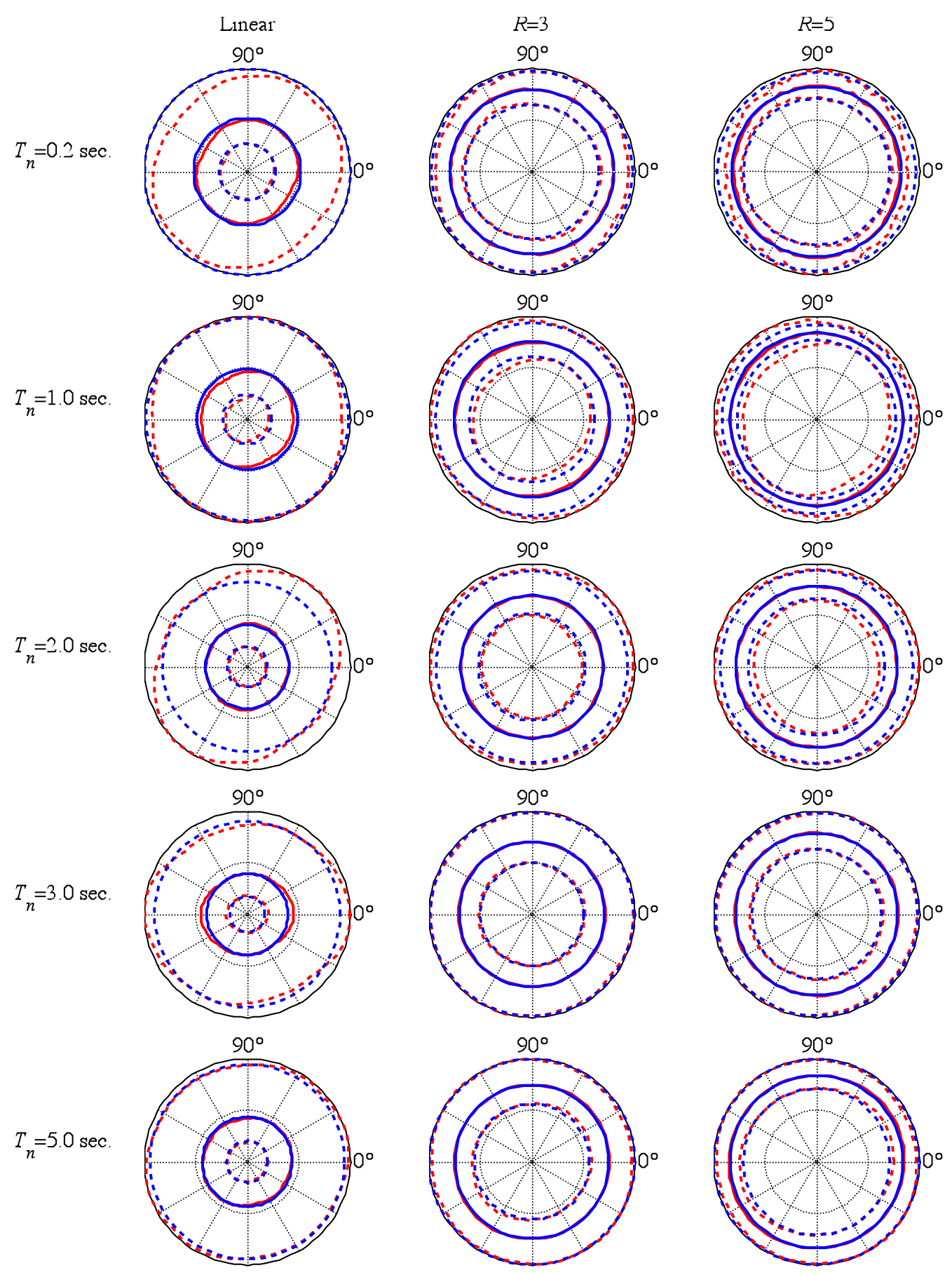

Median brace force $+/$ - one standard deviation due to components rotated $\theta_{x}$ degrees E-:-2: Median brace force $+/$ - one standard deviation due to MD components

Figure 41. Median force, $q_{1}$, in bracing b3 as a function of the rotation angle, $\theta_{x}$, for single-story asymmetric-plan systems with elastic first-mode vibration period $\left(T_{n}\right)=0.2,1,2,3$, and 5 seconds subjected to bidirectional loading. The red lines represent the median force, $q_{1} \pm \sigma$. The blue circles represent the median force, $q_{1} \pm \sigma$, for the systems subjected to bidirectional ground motions in the maximum direction. Solid lines are 50th and dashed lines are16th and 84th percentile engineering demand parameters (EDPs). MD, maximum direction. 

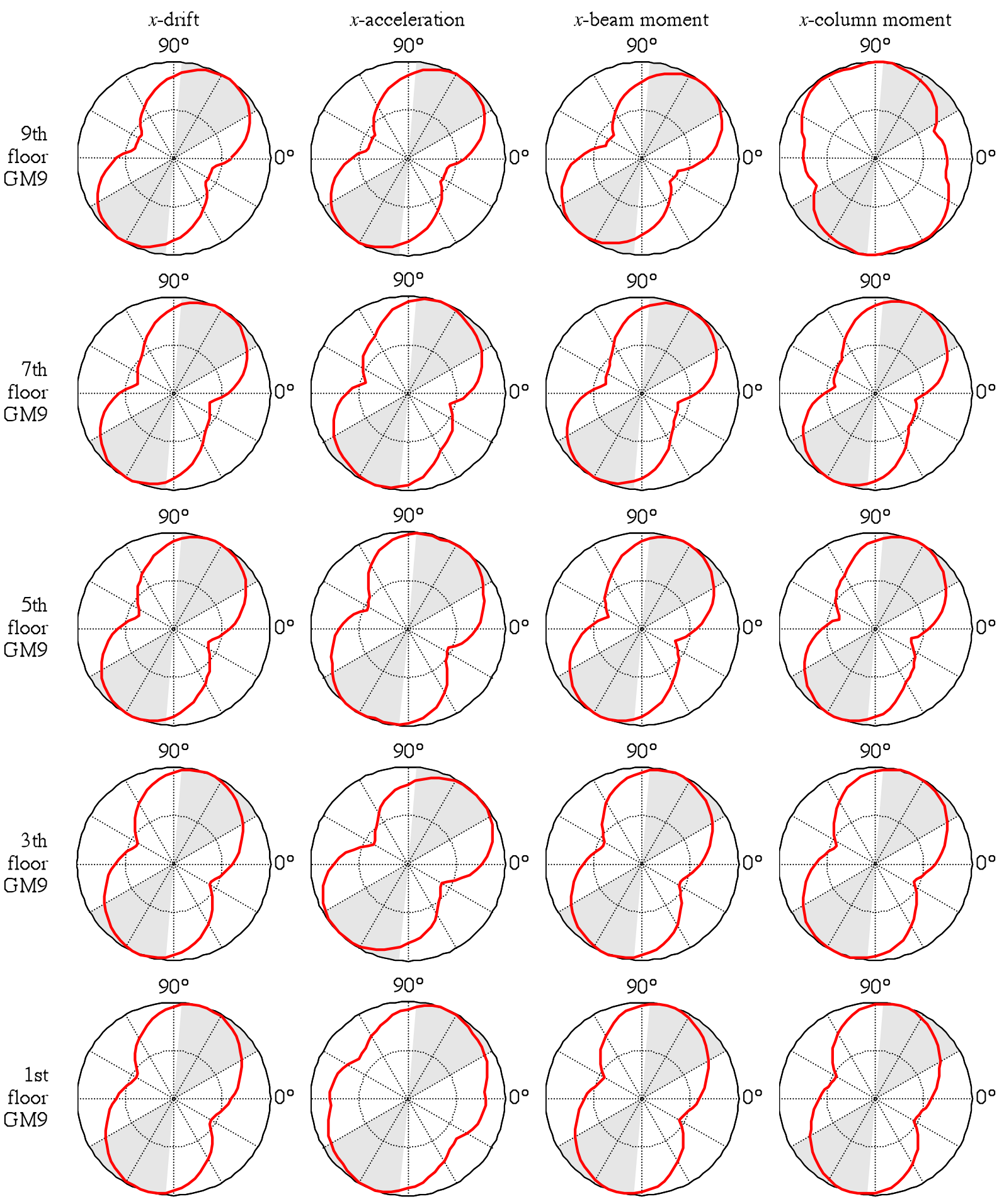

Figure 42. Story drifts, floor total accelerations, and internal forces as a function of the rotation angle, $\theta_{x}$, for the linear nine-story symmetric-plan building (first-mode elastic vibration period, $T_{1}=1.51$ seconds) subjected to ground-motion (GM) no. 9, which has maximum velocity pulse period of 1.9 seconds. The filled gray area shows values of $\theta_{x}$ in which velocity pulses are identified. Angles $\theta_{x}=0^{\circ}$ and $90^{\circ}$ correspond to the fault-parallel and fault-normal directions, respectively. 

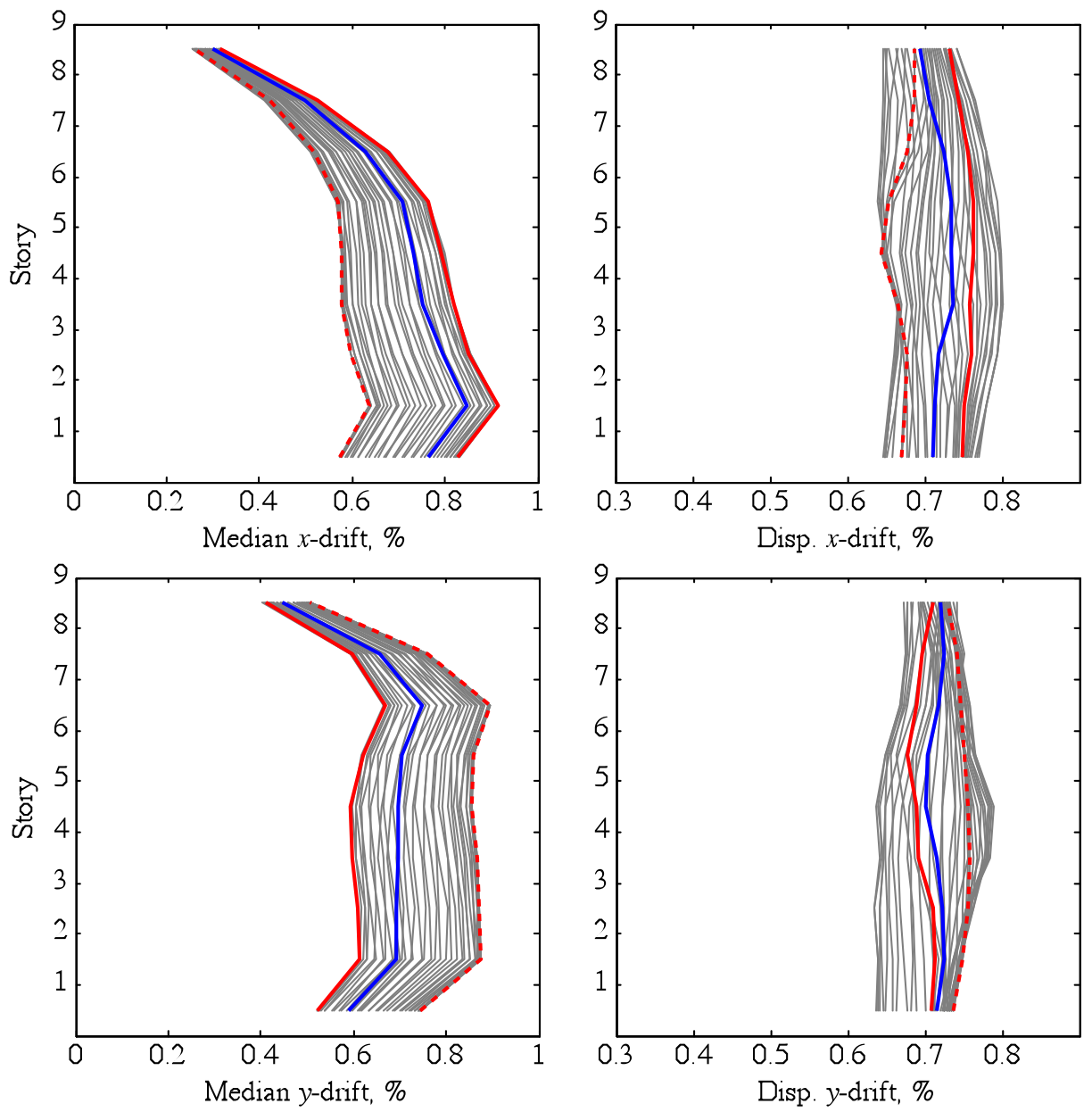

Story drift due to components rotated $\theta_{x}$ degrees

- -.. Story drift due to FN-FP components

Story drift due to MD components

Figure 43. Height-wise distribution of median and dispersion values of story drift in the $x$ and $y$ directions for the linear nine-story symmetric-plan building. The gray, red, and blue lines show median and dispersion values of story drift due to bidirectional ground motions in arbitrary orientations in the faultparallel and fault-normal directions (FN-FP) and in the maximum direction (MD), respectively. \%, percent; disp., displacement. 

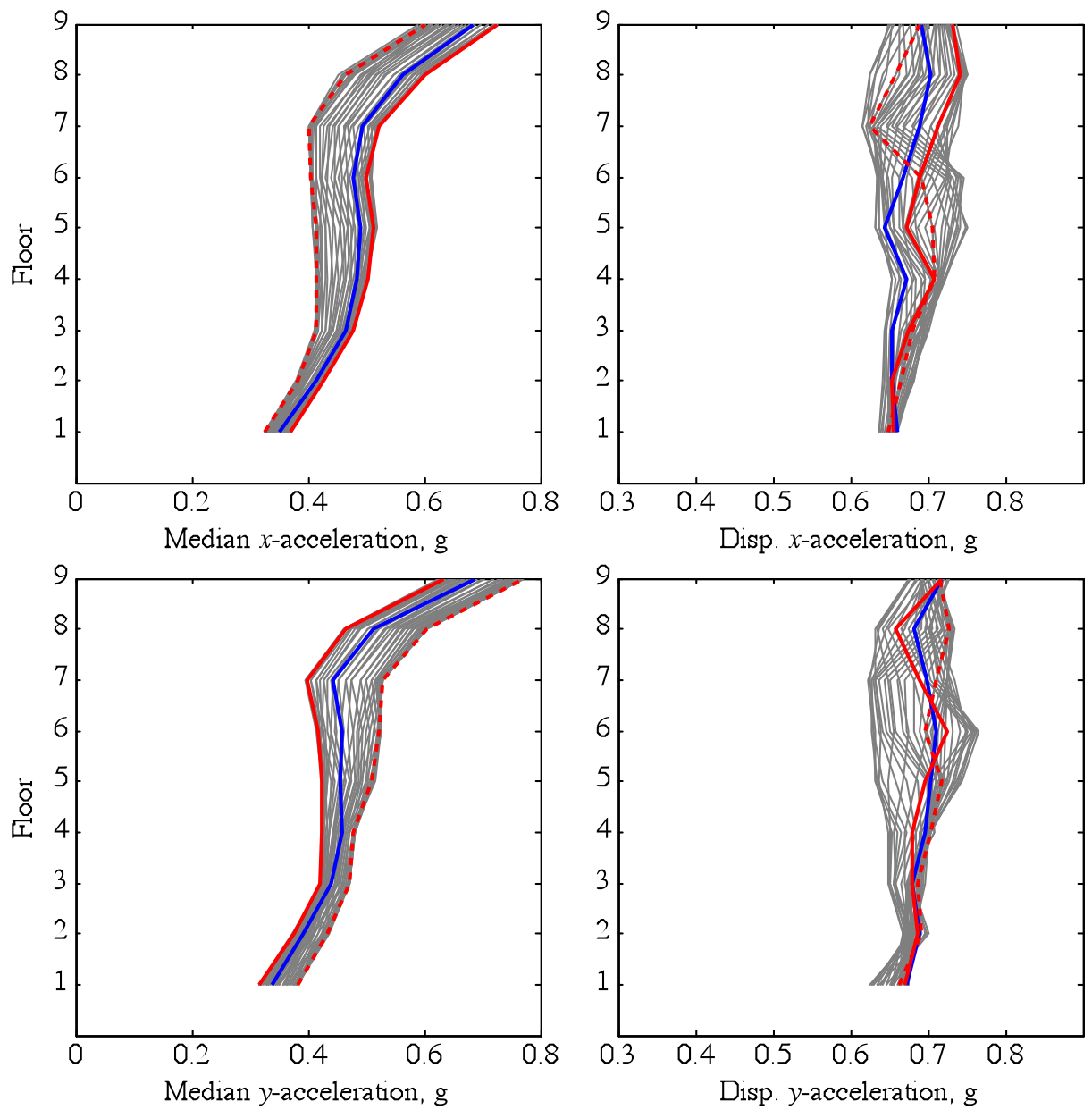

Floor acceleration due to components rotated $\theta_{x}$ degrees

-... Floor acceleration due to FN-FP components

_ Floor acceleration due to MD components

Figure 44. Height-wise distribution of median and dispersion values of floor total acceleration in the $x$ and $y$ directions for the linear nine-story symmetric-plan building. The gray, red, and blue lines show median and dispersion values of floor total accelerations due to bidirectional ground motions in arbitrary orientations in the fault-parallel and fault-normal directions (FN-FP) and in the maximum direction (MD), respectively. \%, percent; disp., displacement. 

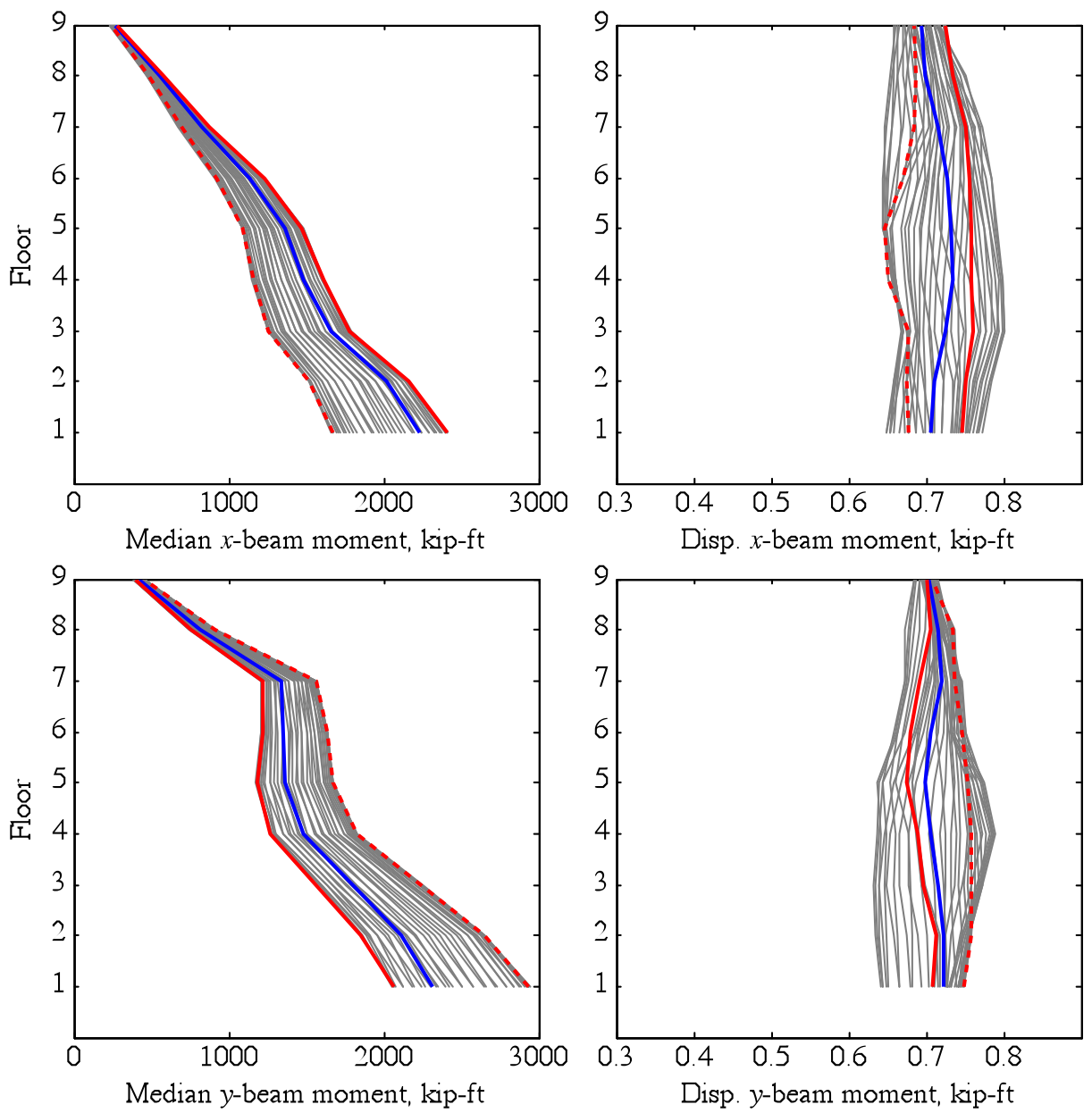

- Beam moment due to components rotated $\theta_{x}$ degrees

_... Beam moment due to FN-FP components

Beam moment due to MD components

Figure 45. Height-wise distribution of median and dispersion values of moments at the beams highlighted in figure 13 for the linear nine-story symmetric-plan building. The gray, red, and blue lines show median and dispersion values of beam moments due to bidirectional ground motions in arbitrary orientations in the fault-parallel and fault-normal directions (FN-FP) and in the maximum direction (MD), respectively. \%, percent; disp., displacement. 

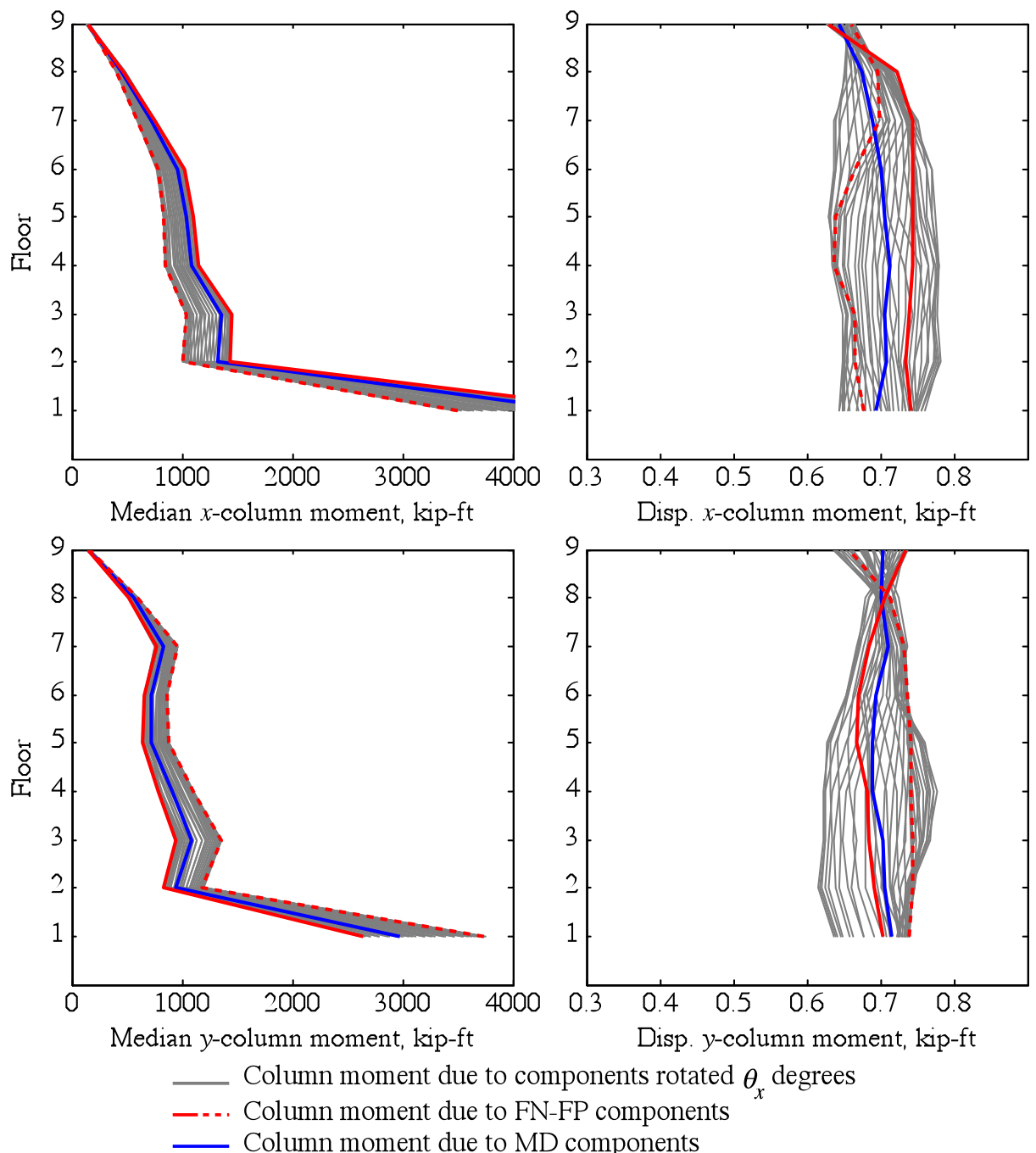

Figure 46. Height-wise distribution of median and dispersion values of moments at the columns highlighted in figure 13 for the linear nine-story symmetric-plan building. The gray, red, and blue lines show median and dispersion values of beam moments due to bidirectional ground motions in arbitrary orientations in the fault-parallel and fault-normal directions (FN-FP) and in the maximum direction (MD), respectively. $\%$, percent; disp., displacement. 

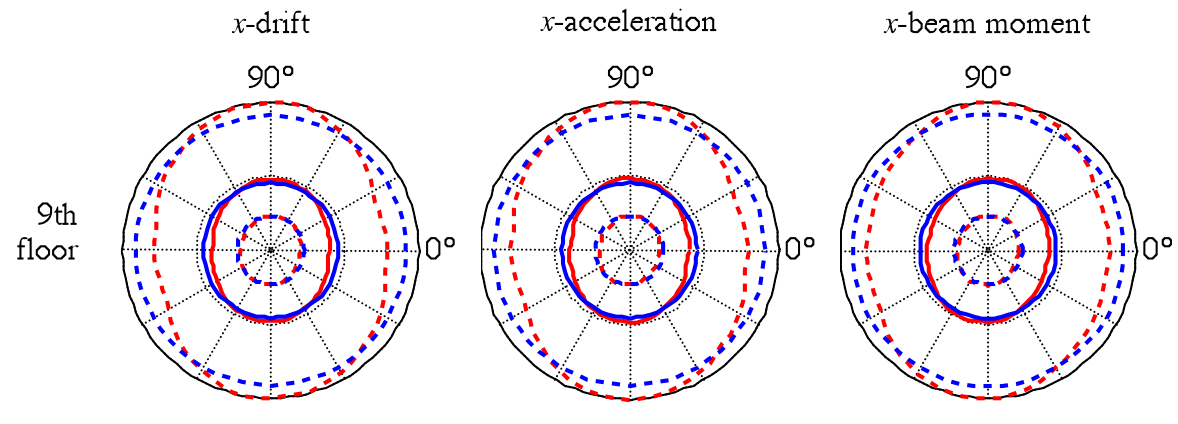

$x$-column moment
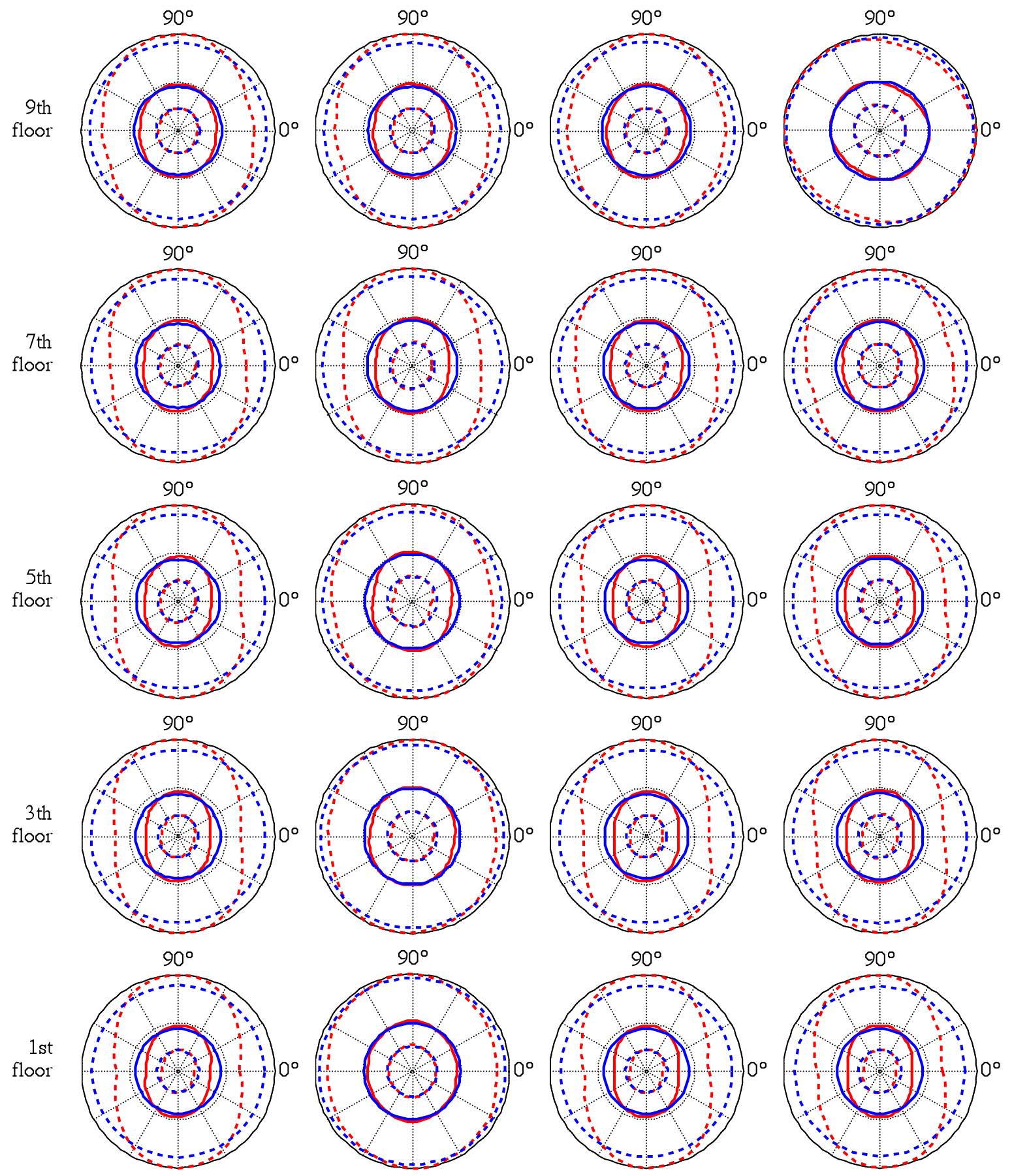

Figure 47. Median values of story drifts, floor total accelerations, and internal forces in the $x$ direction as a function of the rotation angle, $\theta_{x}$, for the linear nine-story symmetric-plan building subjected to bidirectional loading. The red lines represent the median engineering demand parameter (EDP) values $\pm \sigma$. The blue circles represent the median EDP values $\pm \sigma$ for the building subjected to bidirectional ground motions in the maximum direction. Solid lines are 50th and dashed lines are16th and 84th percentile EDPs. 


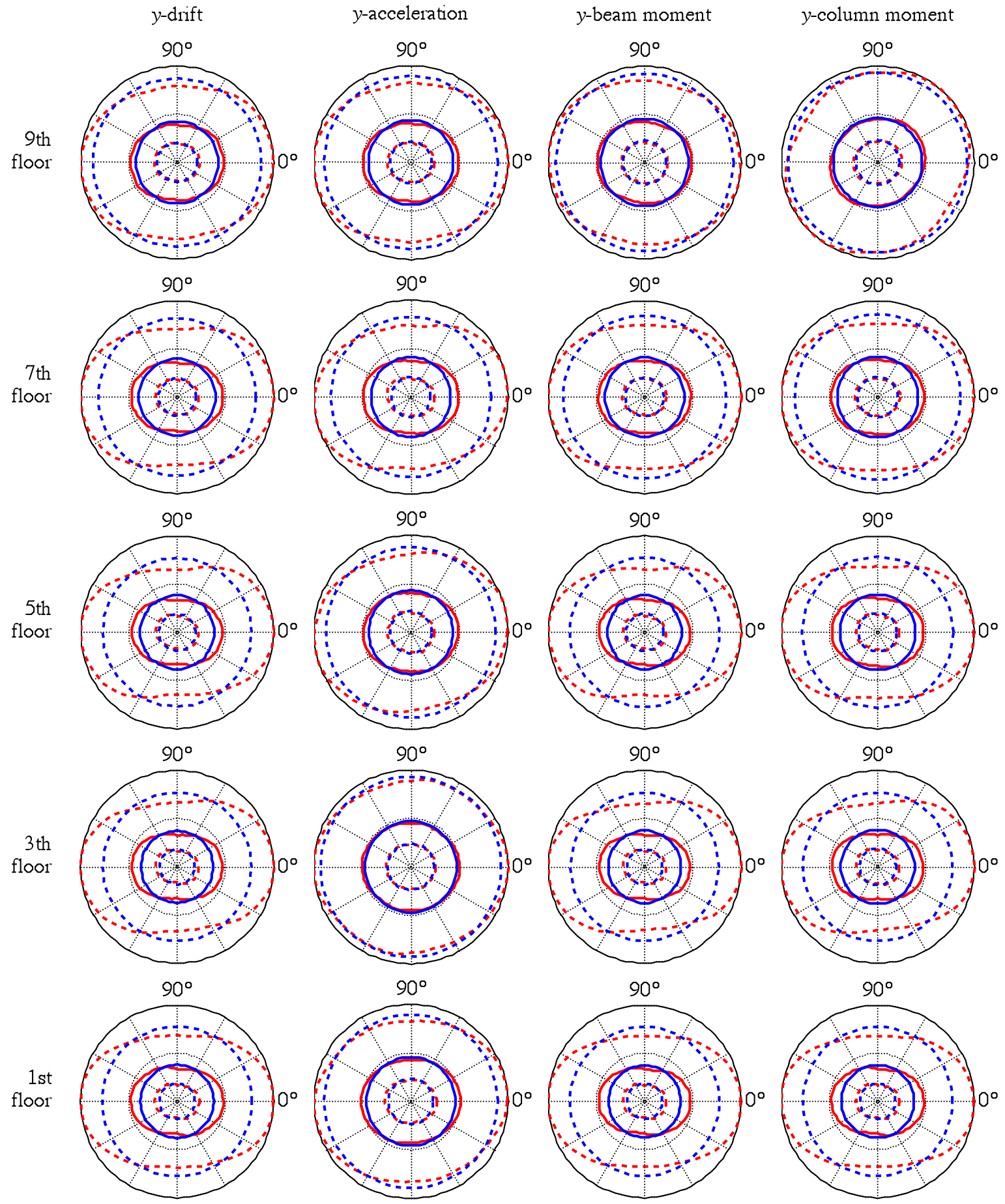

Figure 48. Median values of story drifts, floor total accelerations, and internal forces in the $y$ direction as a function of the rotation angle, $\theta_{x}$, for the linear nine-story symmetric-plan building subjected to bidirectional loading. The red lines represent the median engineering demand parameter (EDP) values $\pm \sigma$. The blue circles represent the median EDP values $\pm \sigma$ for the building subjected to bidirectional ground motions in the maximum direction. Solid lines are 50th and dashed lines are16th and 84th percentile EDPs. 

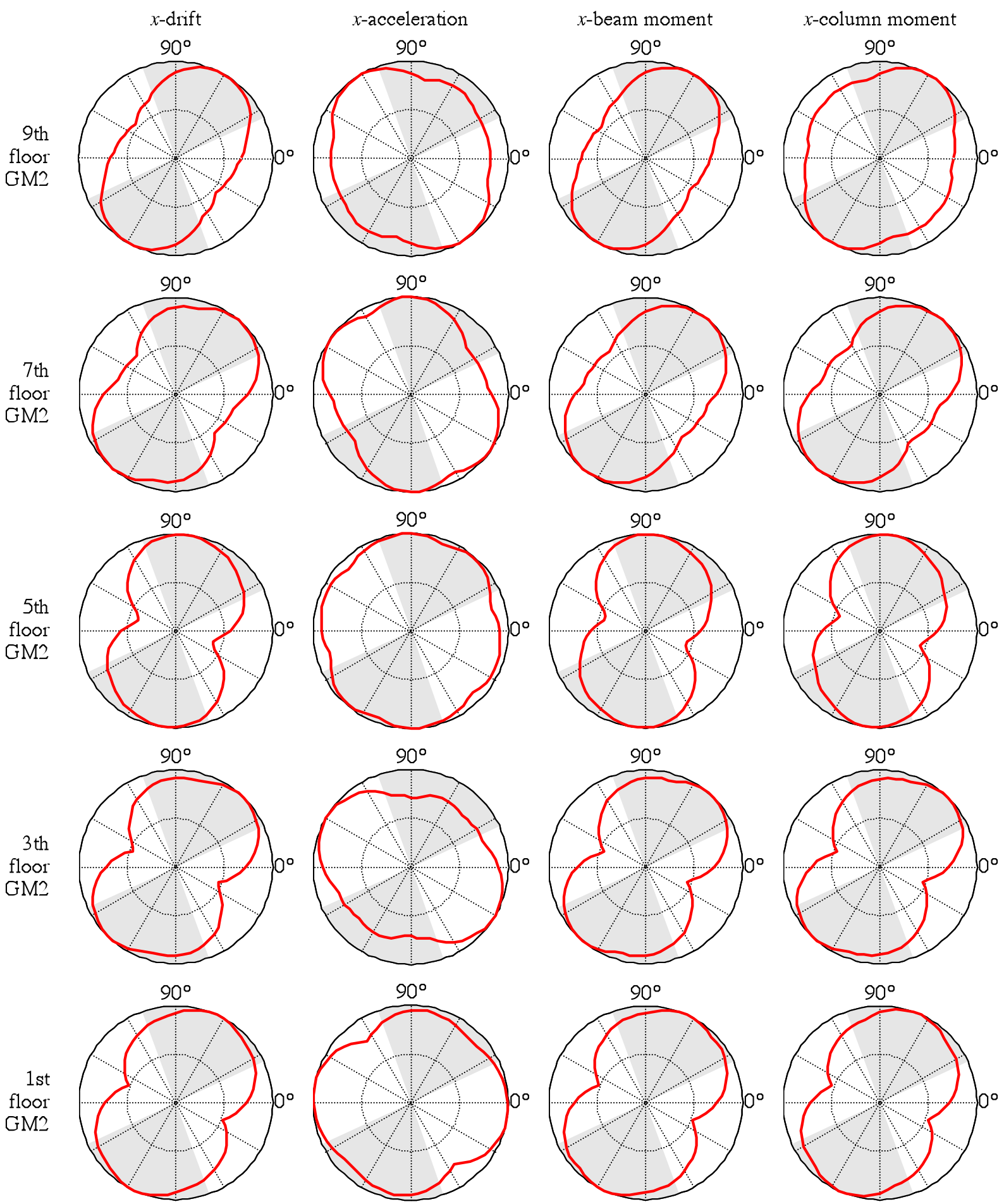

Figure 49. Story drifts, floor total accelerations, and internal forces as a function of the rotation angle, $\theta_{x}$, for the linear nine-story asymmetric-plan building (elastic first-mode vibration period, $T_{1}=2.5$ seconds) subjected to ground motion (GM) 2, which has maximum velocity pulse period of 2.4 seconds. The filled gray area shows values of $\theta_{x}$ in which velocity pulses are identified. Angles $\theta_{x}=0^{\circ}$ and $90^{\circ}$ correspond to the fault-parallel and fault-normal directions, respectively. 

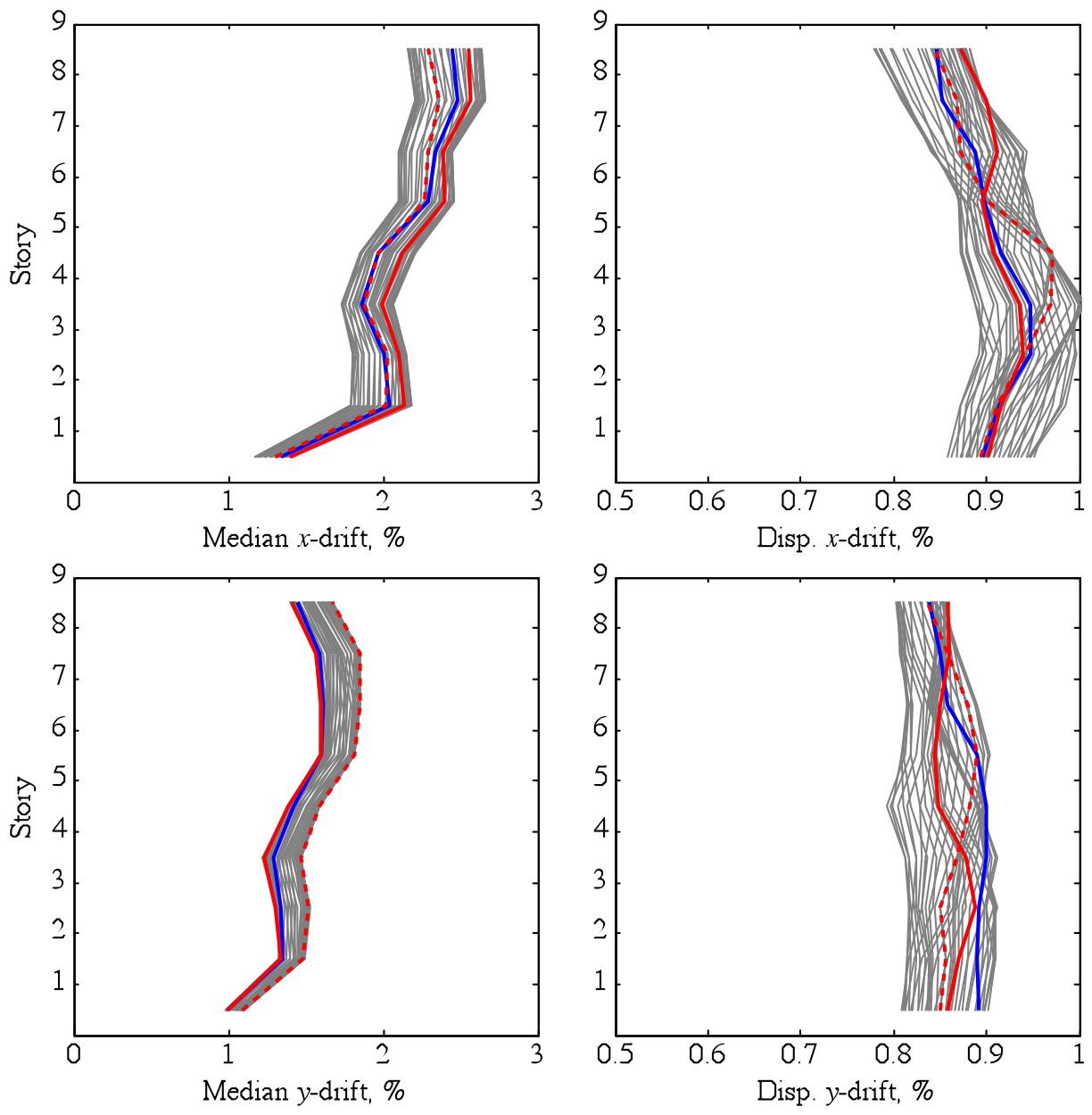

Story drift due to components rotated $\theta_{x}$ degrees

- -.. Story drift due to FN-FP components

Story drift due to MD components

Figure 50. Height-wise distribution of median and dispersion values of story drift at the corner highlighted in figure 20 in the $x$ and $y$ directions for the linear nine-story asymmetric-plan building. The gray, red, and blue lines show median and dispersion values of story drift due to bidirectional ground motions in arbitrary orientations in the fault-parallel and fault-normal directions (FN-FP) and in the maximum direction (MD), respectively. \%, percent; disp., displacement. 

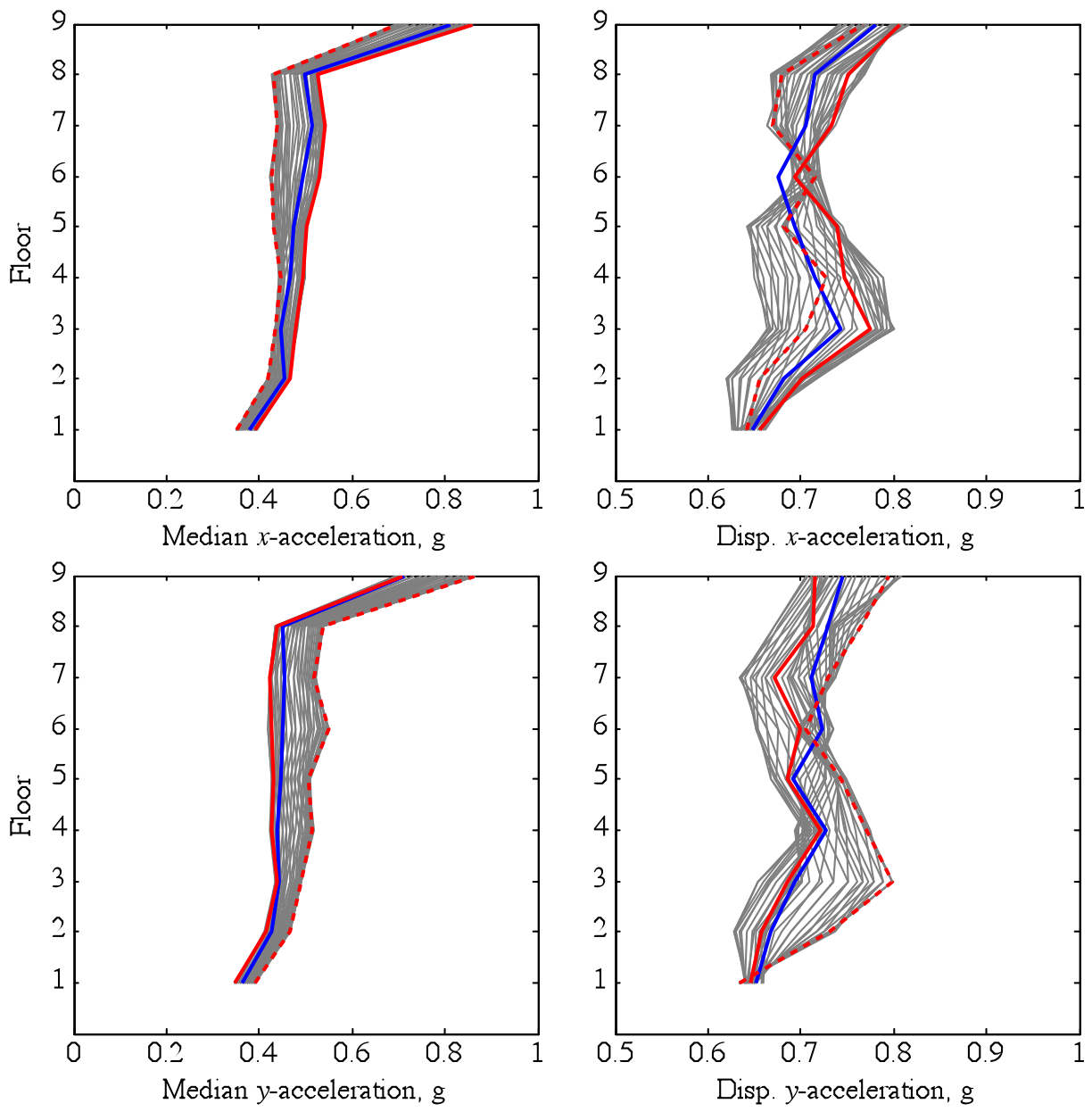

Floor acceleration due to components rotated $\theta_{x}$ degrees

-... Floor acceleration due to FN-FP components

Floor acceleration due to MD components

Figure 51. Height-wise distribution of median and dispersion values of floor total acceleration in the $x$ and $y$ directions for the linear nine-story asymmetric-plan building. The gray, red, and blue lines show median and dispersion values of floor total accelerations due to bidirectional ground motions in arbitrary orientations in the fault-parallel and fault-normal directions (FN-FP) and in the maximum direction (MD), respectively. \%, percent; disp., displacement. 

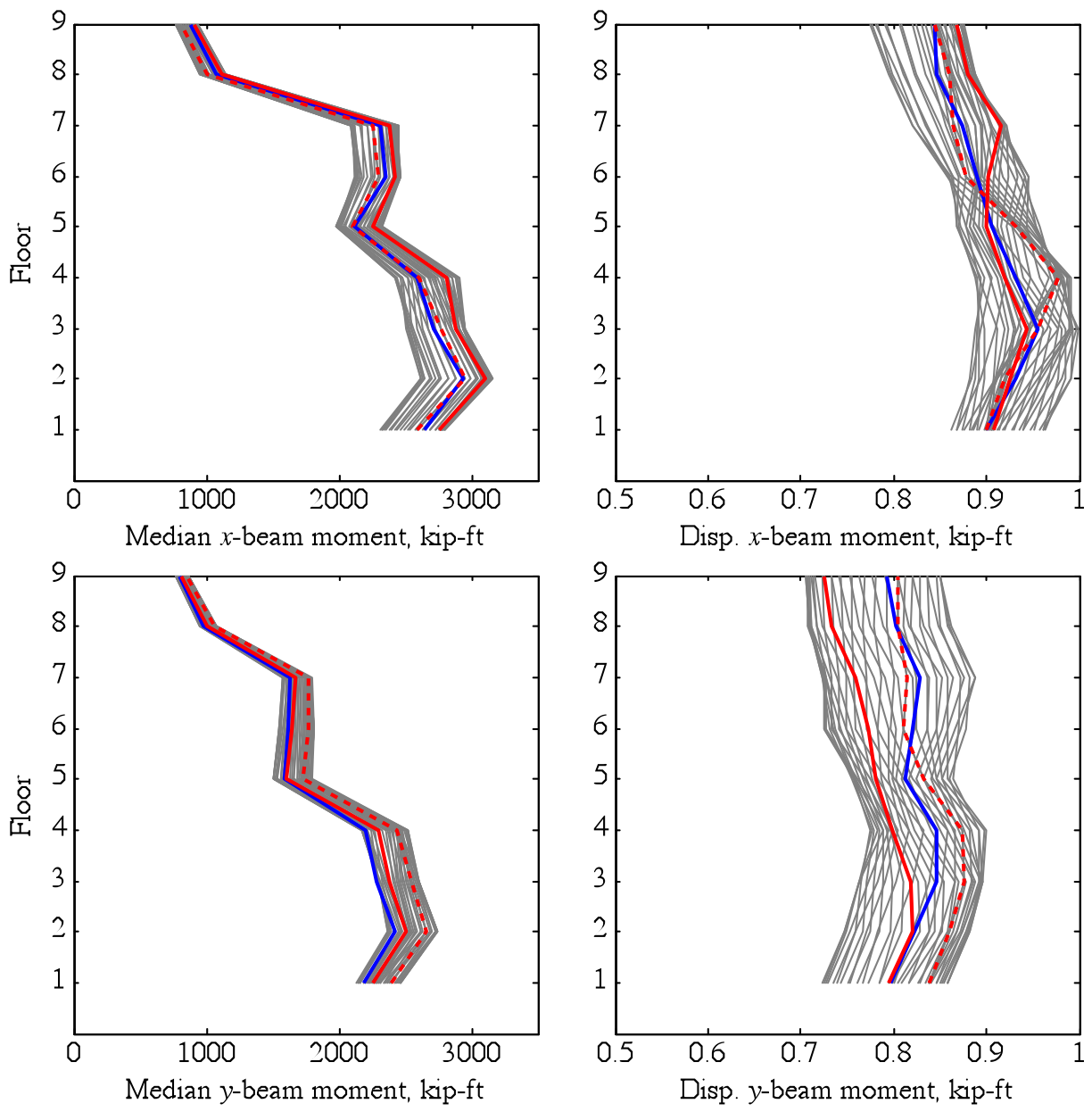

_ Beam moment due to components rotated $\theta_{x}$ degrees

-... Beam moment due to FN-FP components

_ Beam moment due to MD components

Figure 52. Height-wise distribution of median and dispersion values of moments at the beams highlighted in figure 20 for the linear nine-story asymmetric-plan building. The gray, red, and blue lines show median and dispersion values of beam moments due to bidirectional ground motions in arbitrary orientations in the fault-parallel and fault-normal directions (FN-FP) and in the maximum direction (MD), respectively. \%, percent; disp., displacement. 

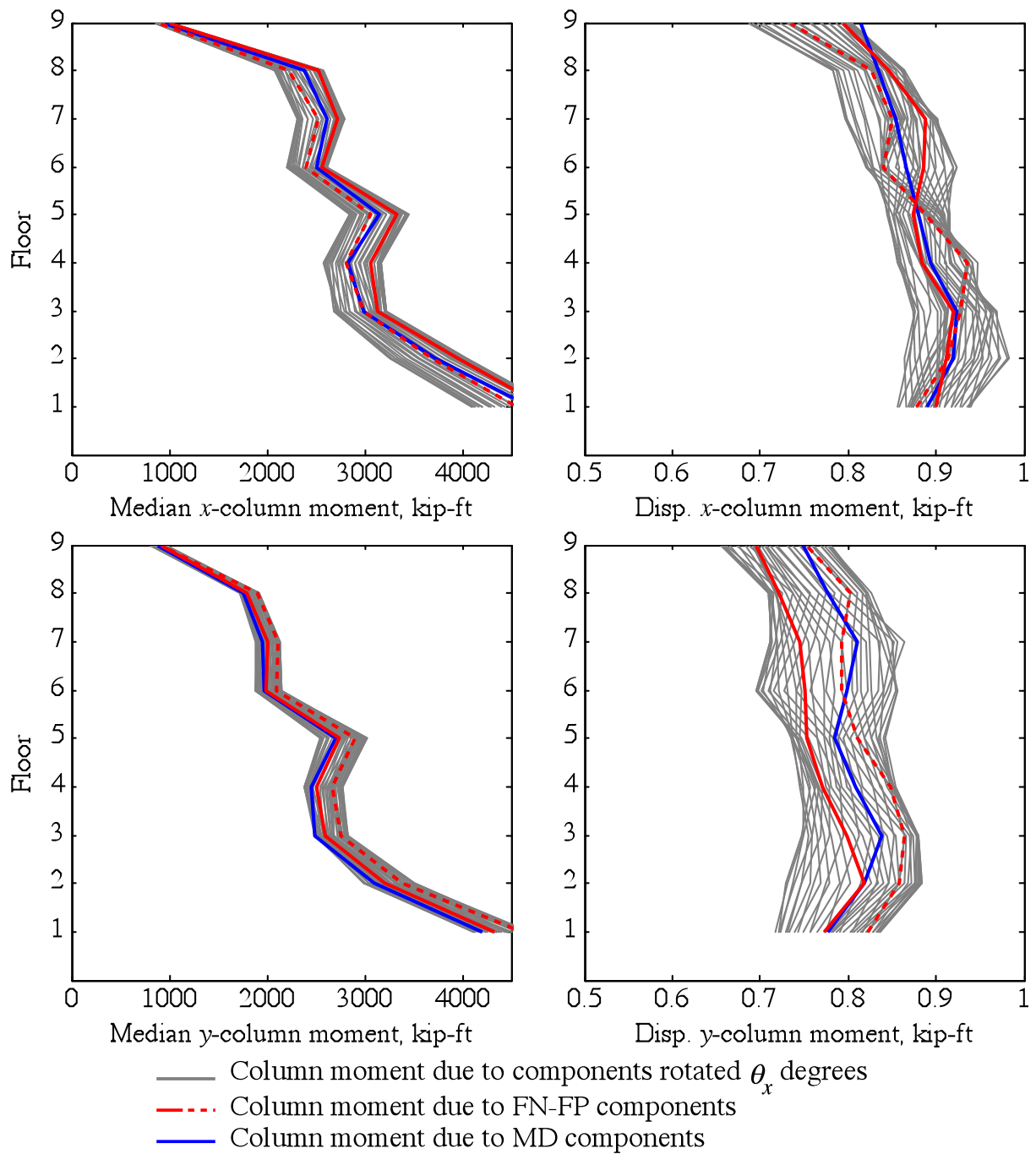

Figure 53. Height-wise distribution of median and dispersion values of moments at the columns highlighted in figure 20 for the linear nine-story asymmetric-plan building. The gray, red, and blue lines show median and dispersion values of beam moments due to bidirectional ground motions in arbitrary orientations in the fault-parallel and fault-normal directions (FN-FP) and in the maximum direction (MD), respectively. \%, percent; disp., displacement. 

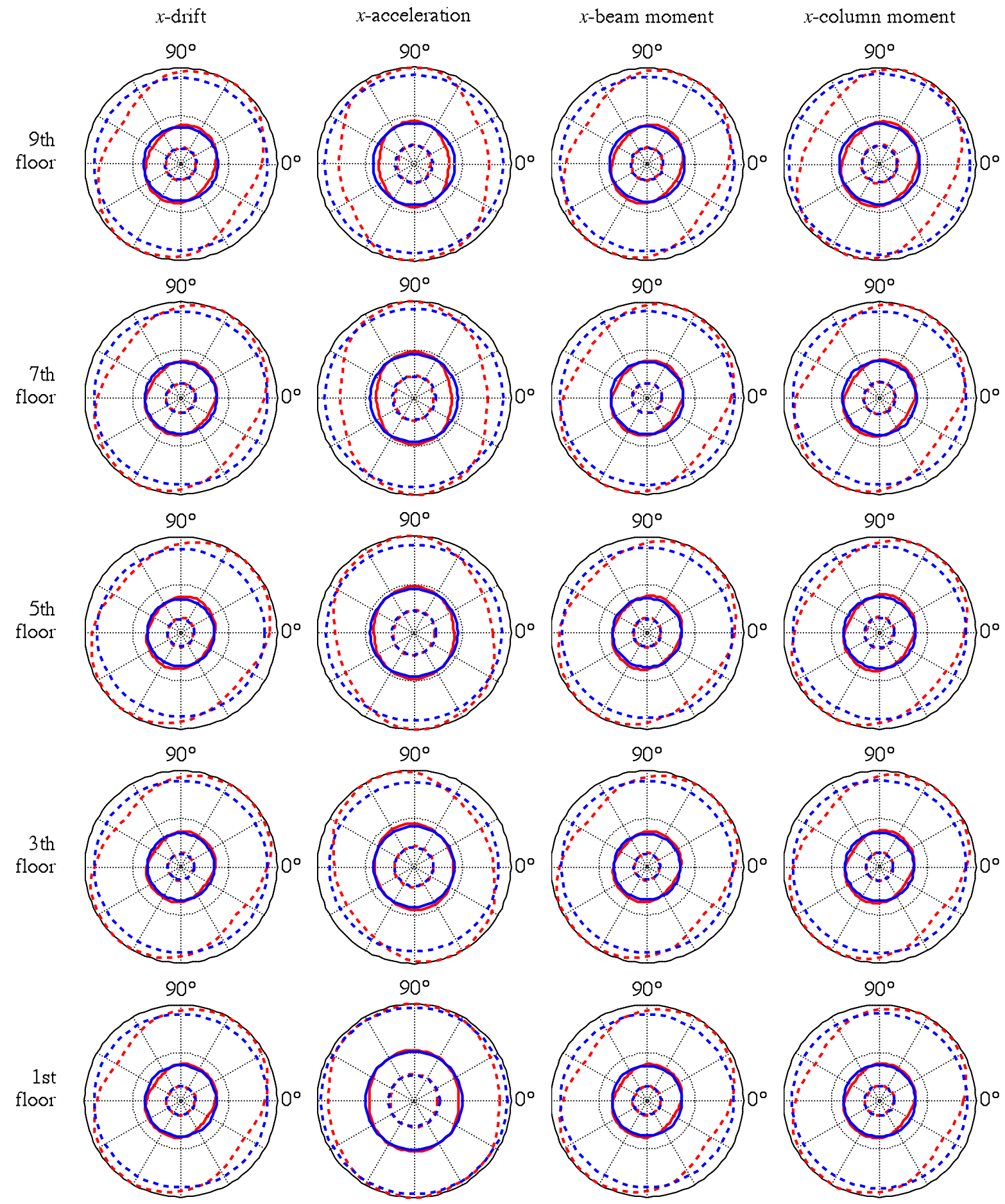

Figure 54. Median values of story drifts at the corner, floor total accelerations, and internal forces in the $x$ direction as a function of the rotation angle, $\theta_{x}$, for the linear nine-story asymmetric-plan building subjected to bidirectional loading. The red lines represent the median engineering demand parameter $(E D P)$ values $\pm \sigma$. The blue circles represent the median EDP values $\pm \sigma$ for the building subjected to bidirectional ground motions in the maximum direction. Solid lines are Solid lines are $50^{\text {th }}$ and dashed lines are $16^{\text {th }}$ and $84^{\text {th }}$ percentile EDPs. 


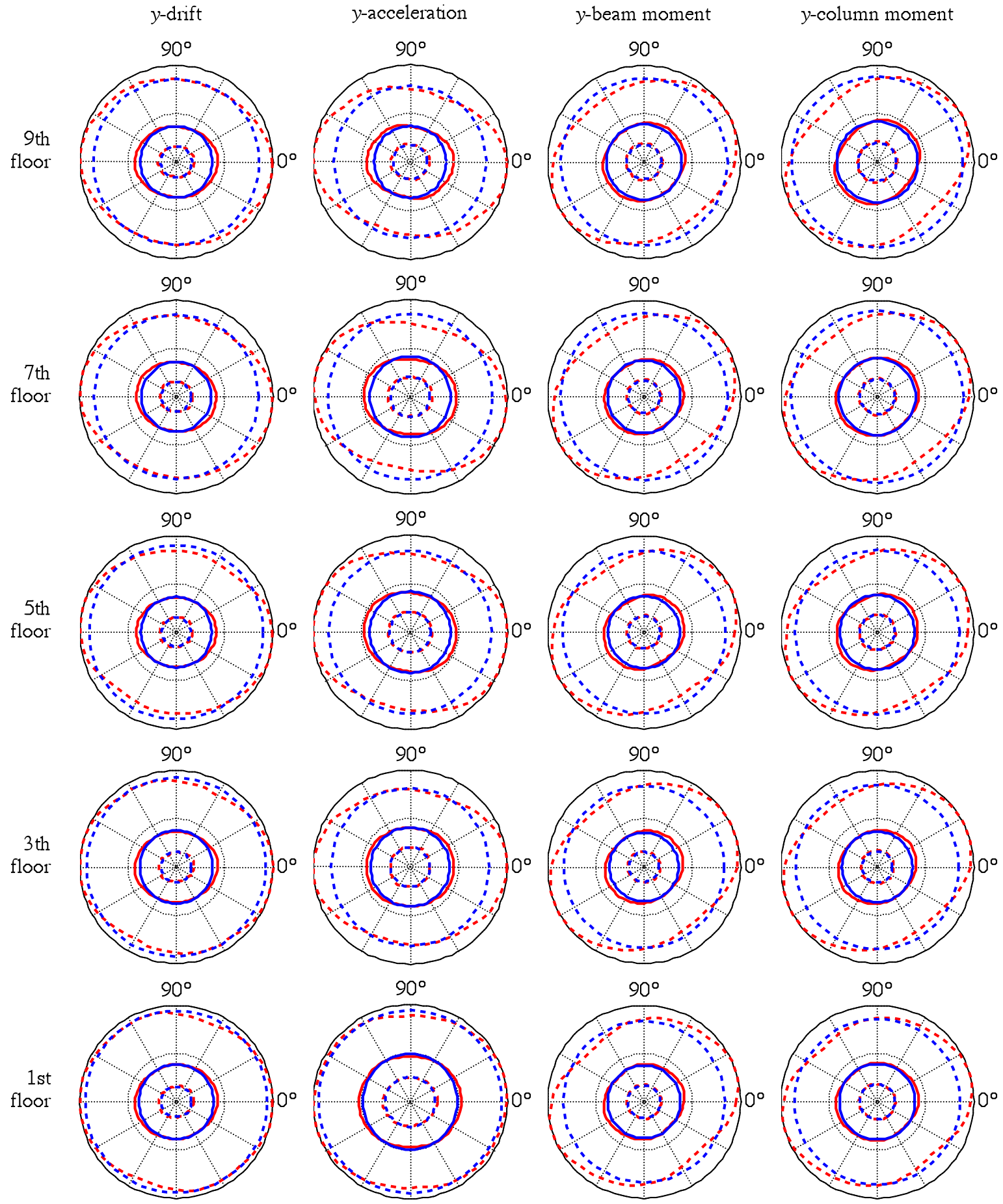

Figure 55. Median values of story drifts, floor total accelerations, and internal forces in the $y$ direction as a function of the rotation angle, $\theta_{\mathrm{x}}$, for the linear nine-story asymmetric-plan building subjected to bidirectional loading. The red lines represent the median engineering demand parameter (EDP) values $\pm \sigma$. The blue circles represent the median EDP values $\pm \sigma$ for the building subjected to bidirectional ground motions in the maximum-direction. Solid lines are $50^{\text {th }}$ and dashed lines are $16^{\text {th }}$ and $84^{\text {th }}$ percentile EDPs. 

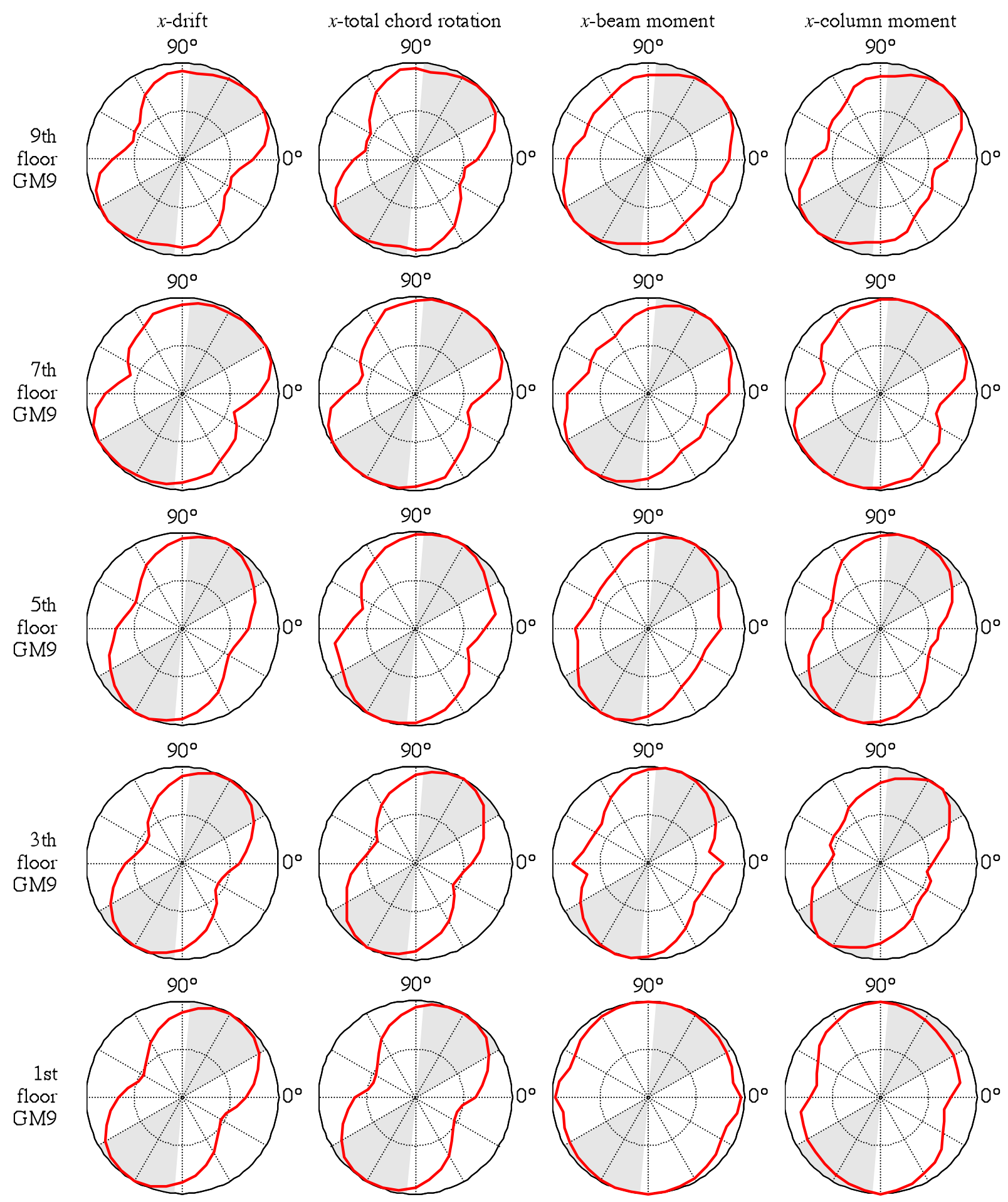

Figure 56. Story drifts, total chord rotations, and internal forces as a function of the rotation angle, $\theta_{x}$, for the nonlinear nine-story symmetric-plan building (first-mode elastic vibration period, $T_{1}=1.51$ seconds) subjected to ground motion (GM) 9 , which has maximum velocity pulse period of 1.9 seconds. The filled gray area shows values of $\theta_{x}$ in which velocity pulses are identified. Angles $\theta_{x}=0^{\circ}$ and $90^{\circ}$ correspond to the fault-parallel and fault-normal directions, respectively. 

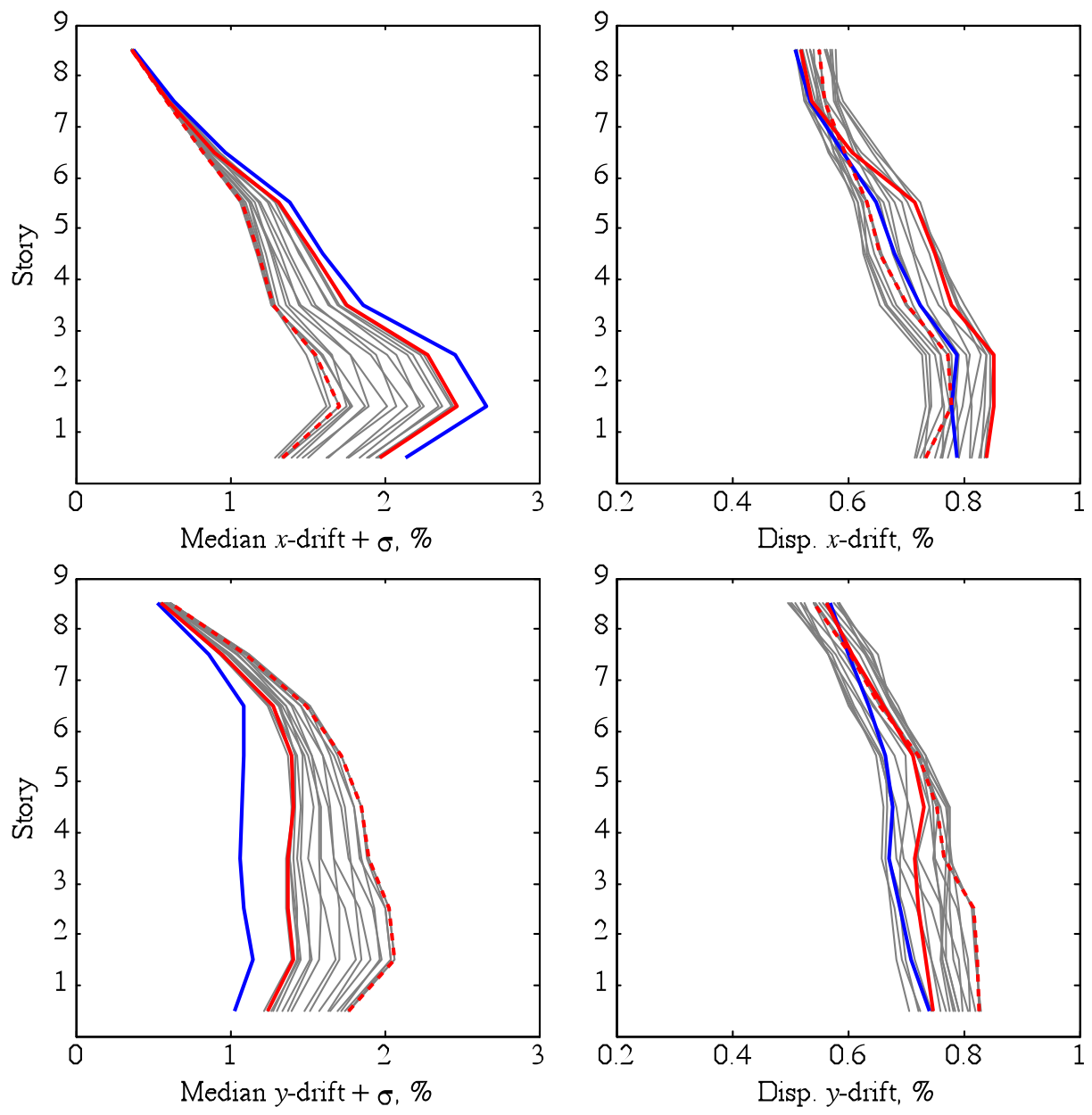

- Story drift due to components rotated $\theta_{x}$ degrees

- ... Story drift due to FN-FP components

- Story drift due to MD components

Figure 57. Height-wise distribution of median $+\sigma$ and dispersion values of story drift in the $x$ and $y$ directions for the nonlinear nine-story symmetric-plan building. The gray, red, and blue lines show median $+\sigma$ and dispersion values of story drift due to bidirectional ground motions in arbitrary orientations in the fault-parallel and fault-normal directions (FN-FP) and in the maximum direction (MD), respectively. \%, percent; disp., displacement. 

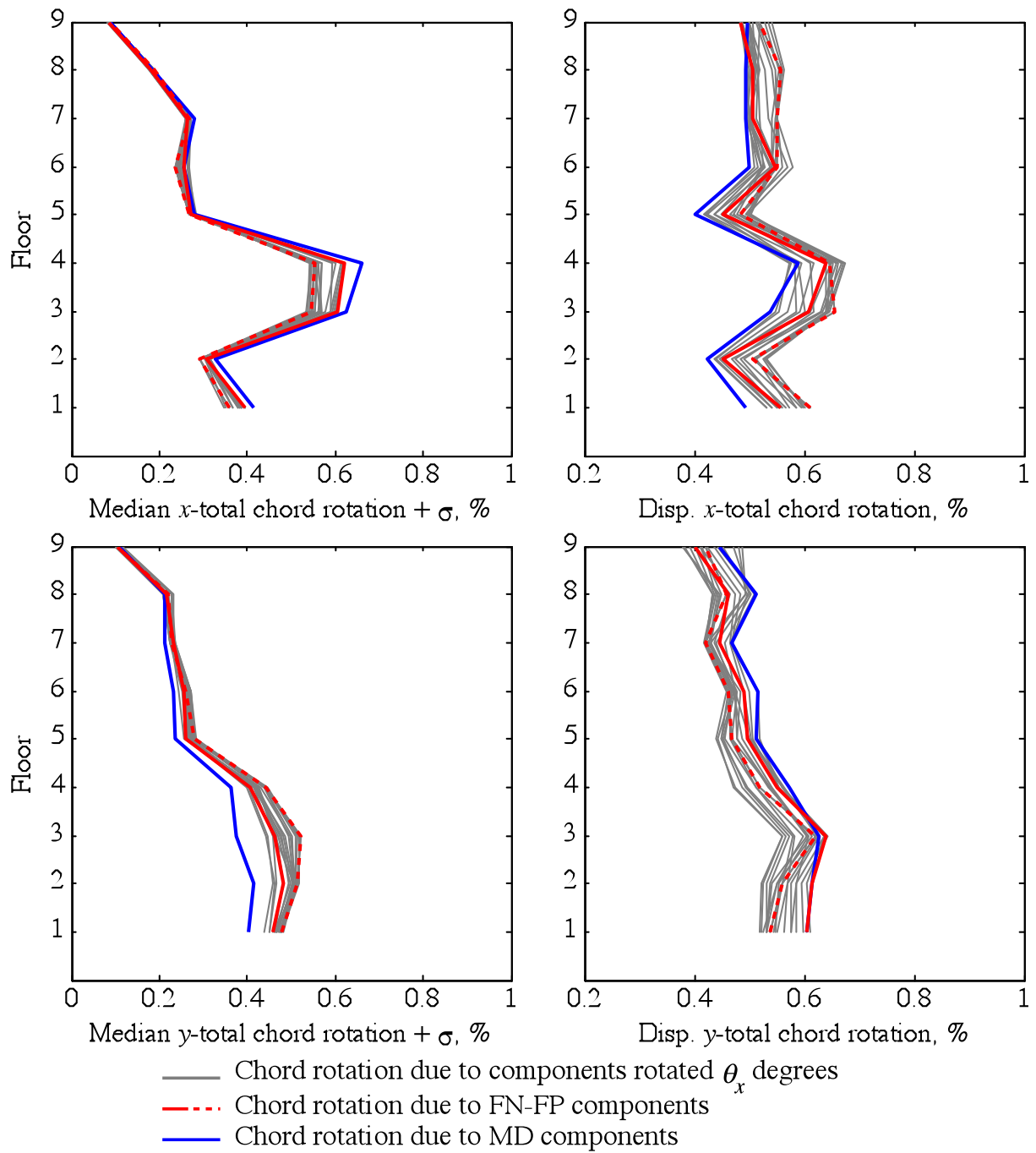

Figure 58. Height-wise distribution of median $+\sigma$ and dispersion values of total chord rotation in the $x$ and $y$ directions for the nonlinear nine-story symmetric-plan building. The gray, red, and blue lines show median $+\sigma$ and dispersion values of floor total accelerations due to bidirectional ground motions in arbitrary orientations in the fault-parallel and fault-normal directions (FN-FP) and in the maximum direction (MD), respectively. \%, percent; disp., displacement. 

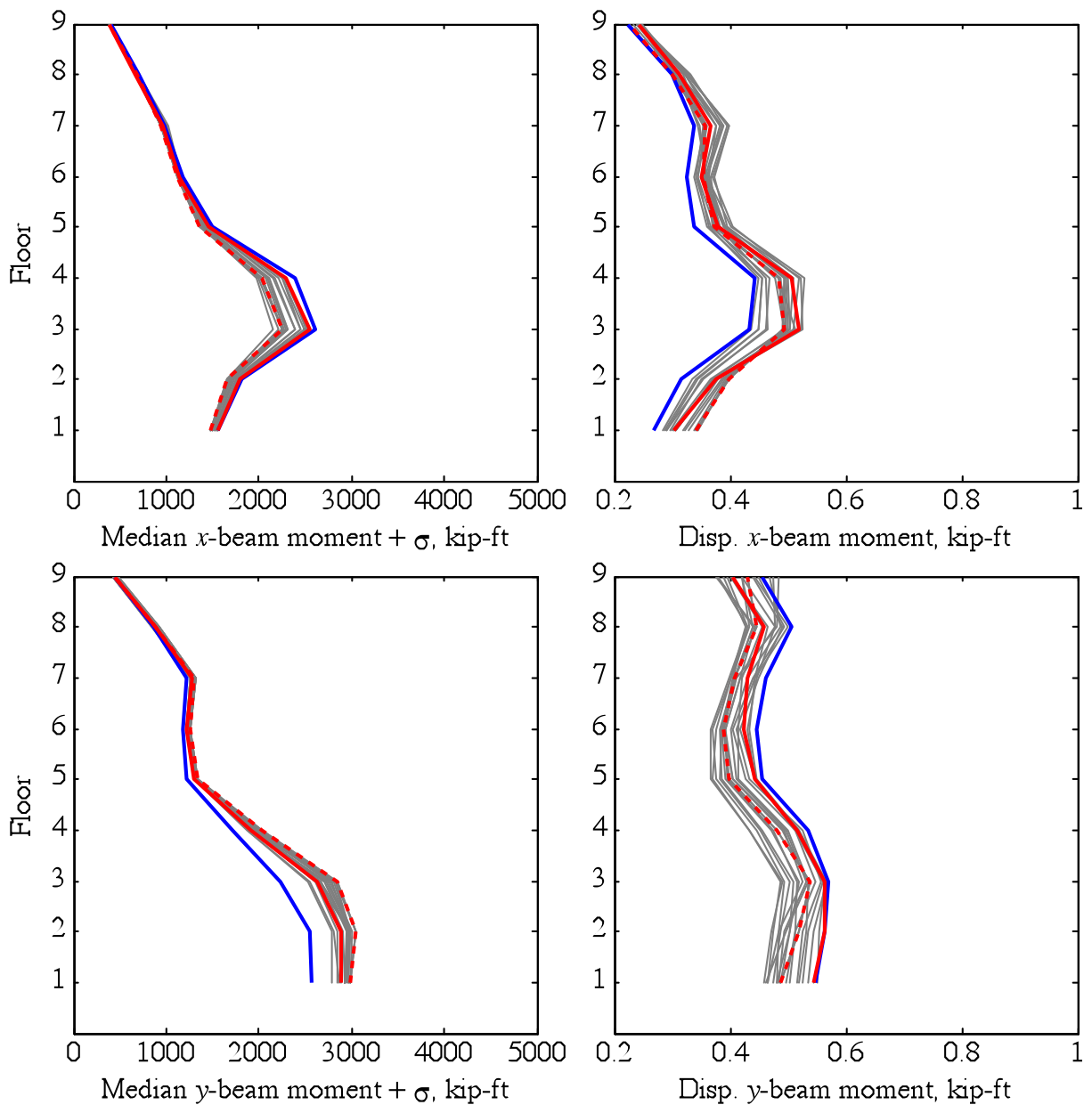

_ Beam moment due to components rotated $\theta_{x}$ degrees

- ... Beam moment due to FN-FP components

Beam moment due to MD components

Figure 59. Height-wise distribution of median $+\sigma$ and dispersion values of moments at the beams highlighted in figure 13 for the nonlinear nine-story symmetric-plan building. The gray, red, and blue lines show median $+\sigma$ and dispersion values of beam moments due to bidirectional ground motions in arbitrary orientations in the fault-parallel and fault-normal directions (FN-FP) and in the maximum direction (MD), respectively. \%, percent; disp., displacement. 

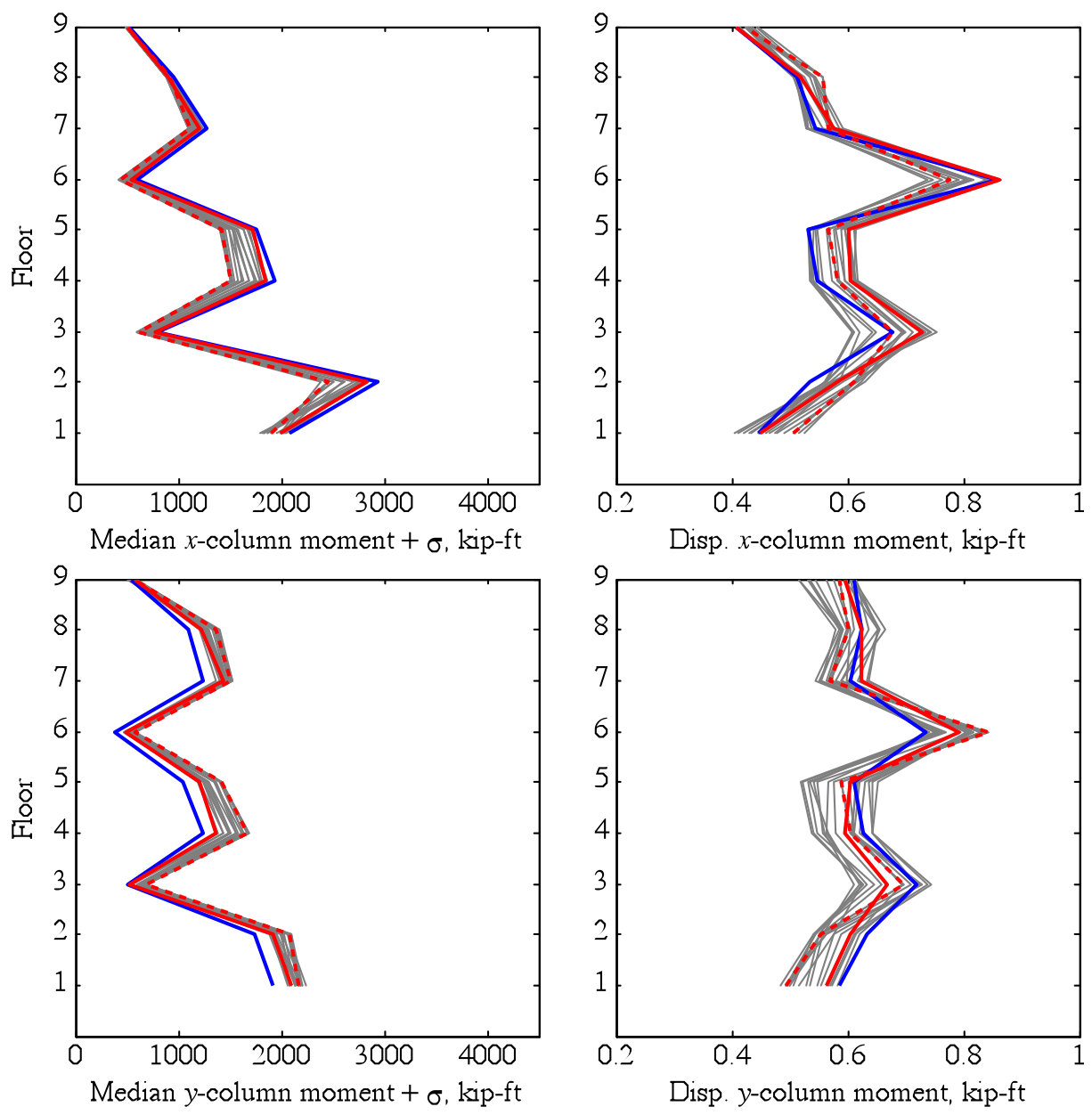

Column moment due to components rotated $\theta_{x}$ degrees

- ... Column moment due to FN-FP components

Column moment due to MD components

Figure 60. Height-wise distribution of median $+\sigma$ and dispersion values of moments at the columns highlighted in figure 13 for the nonlinear nine-story symmetric-plan building. The gray, red, and blue lines show median $+\sigma$ and dispersion values of beam moments due to bidirectional ground motions in arbitrary orientations in the fault-parallel and fault-normal directions (FN-FP) and in the maximum direction (MD), respectively. \%, percent; disp., displacement. 

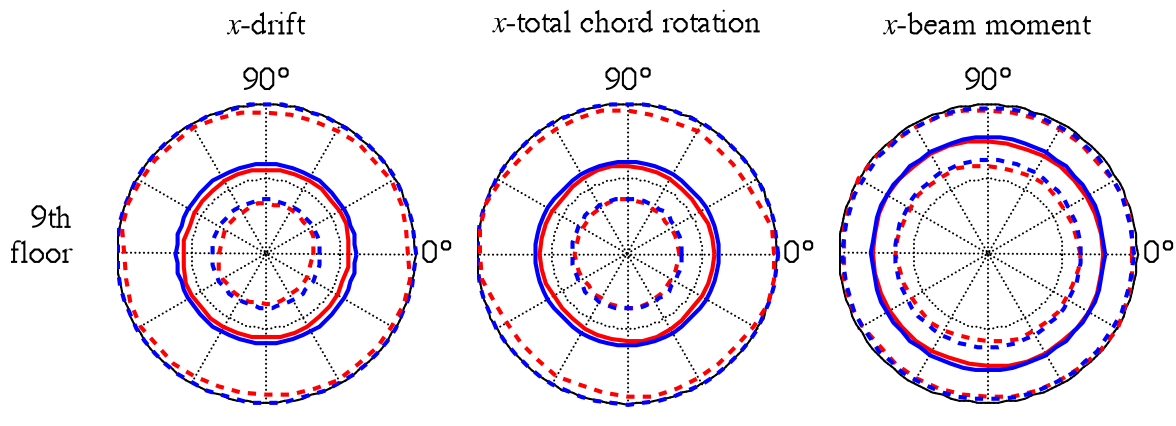

$x$-column moment
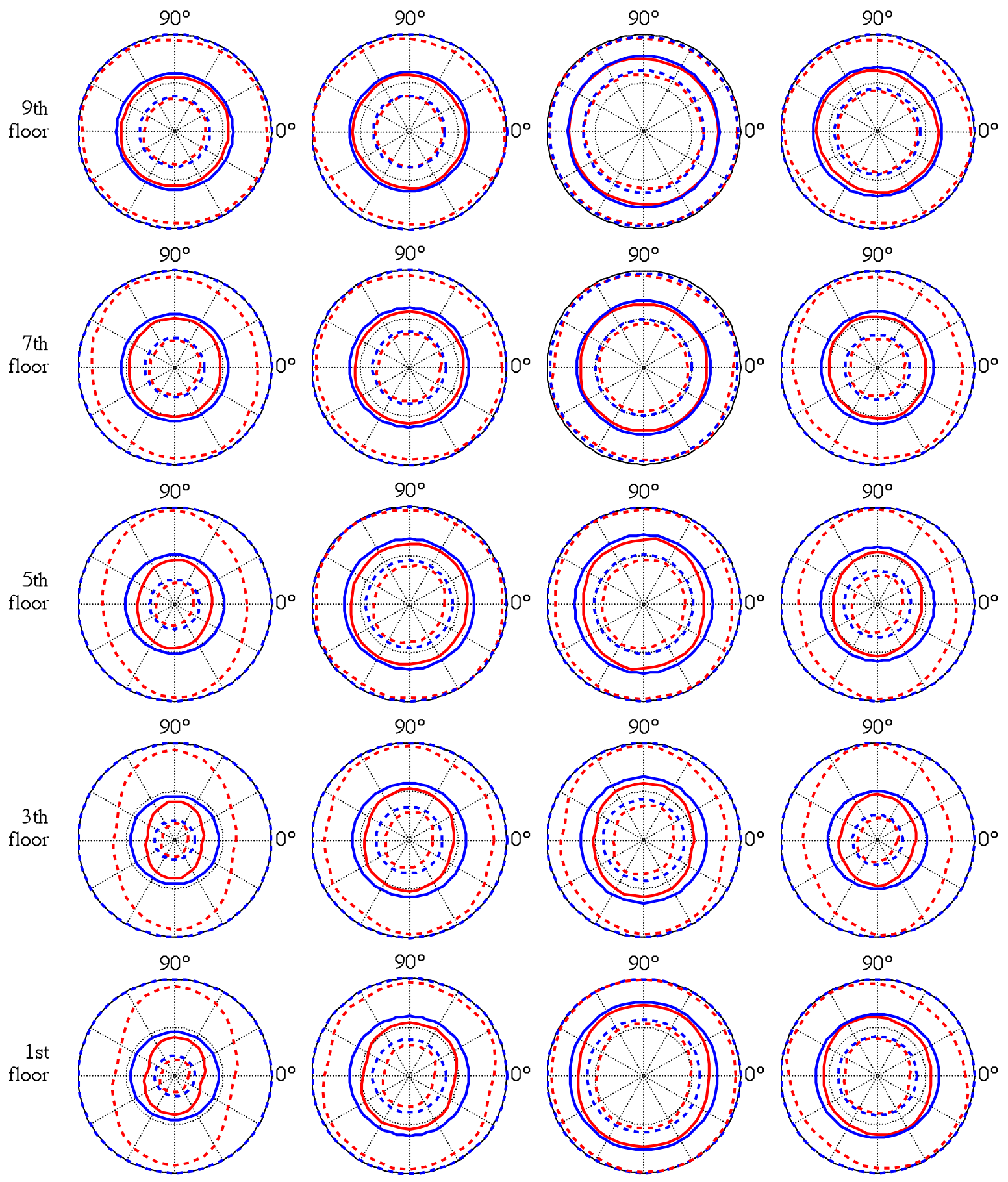

Figure 61. Median values of story drifts, total chord rotations, and internal forces in the $x$ direction as a function of the rotation angle, $\theta_{x}$, for the nonlinear nine-story symmetric-plan building subjected to bidirectional loading. The red lines represent the median engineering demand parameter (EDP) values $\pm \sigma$. The blue circles represent the median EDP values $\pm \sigma$ for the building subjected to bidirectional ground motions in the maximum direction. Solid lines are $50^{\text {th }}$ and dashed lines are $16^{\text {th }}$ and $84^{\text {th }}$ percentile EDPs. 


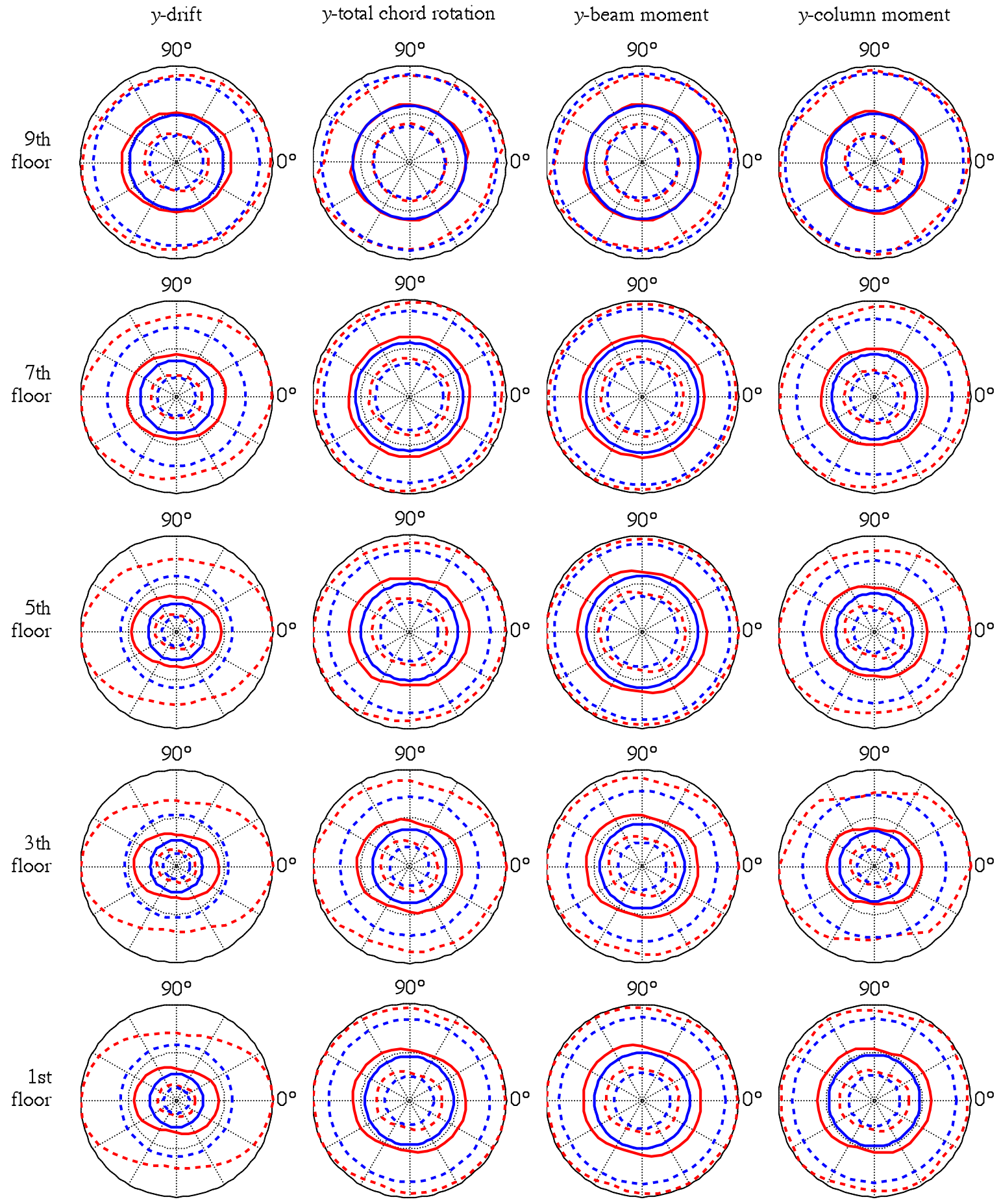

Figure 62. Median values of story drifts, total chord rotations, and internal forces in the $y$ direction as a function of the rotation angle, $\theta_{\mathrm{x}}$, for the nonlinear nine-story symmetric-plan building subjected to bidirectional loading. The red lines represent the median engineering demand parameter (EDP) values $\pm \sigma$. The blue circles represent the median EDP values $\pm \sigma$ for the building subjected to bidirectional ground motions in the maximum direction. Solid lines are $50^{\text {th }}$ and dashed lines are $16^{\text {th }}$ and $84^{\text {th }}$ percentile EDPs. 

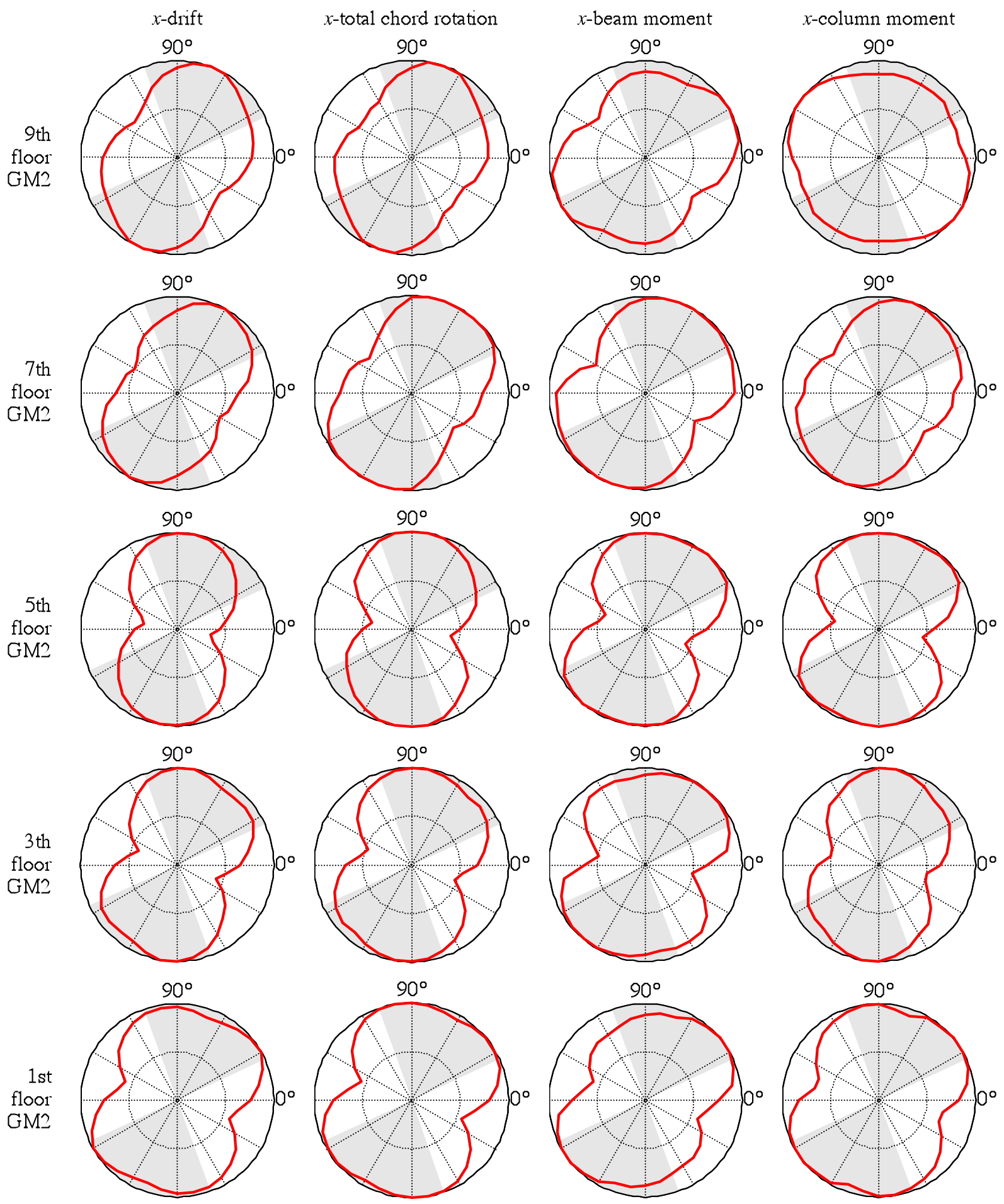

Figure 63. Story drifts, total chord rotations, and internal forces as a function of the rotation angle, $\theta_{x}$, for the nonlinear nine-story asymmetric-plan building $\left(T_{1}=2.5 \mathrm{~s}\right)$ subjected to ground motion 2 , which has maximum velocity pulse period of 2.4 seconds. The filled gray area shows values of $\theta_{x}$ in which velocity pulses are identified. Angles $\theta_{x}=0^{\circ}$ and $90^{\circ}$ correspond to the fault-parallel and fault-normal directions, respectively. 

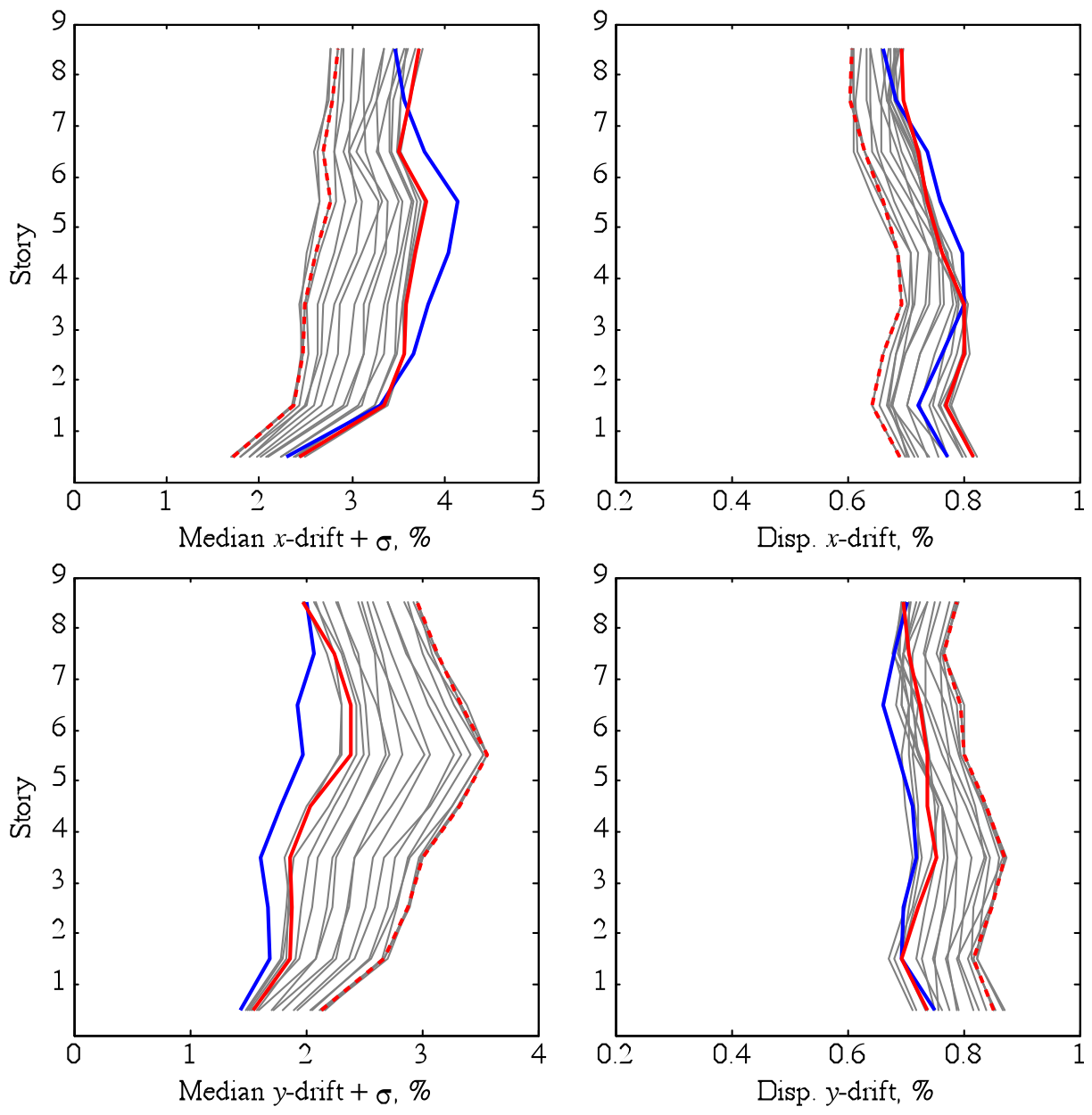

Story drift due to components rotated $\theta_{x}$ degrees

- -.. Story drift due to FN-FP components

Story drift due to MD components

Figure 64. Height-wise distribution of median $+\sigma$ and dispersion values of story drift at the corner highlighted in figure 20 in the $x$ and $y$ directions for the nonlinear nine-story asymmetric-plan building. The gray, red, and blue lines show median $+\sigma$ and dispersion values of story drift due to bidirectional ground motions in arbitrary orientations in the fault-parallel and fault-normal directions (FN-FP) and in the maximum direction (MD), respectively. \%, percent; disp., displacement. 

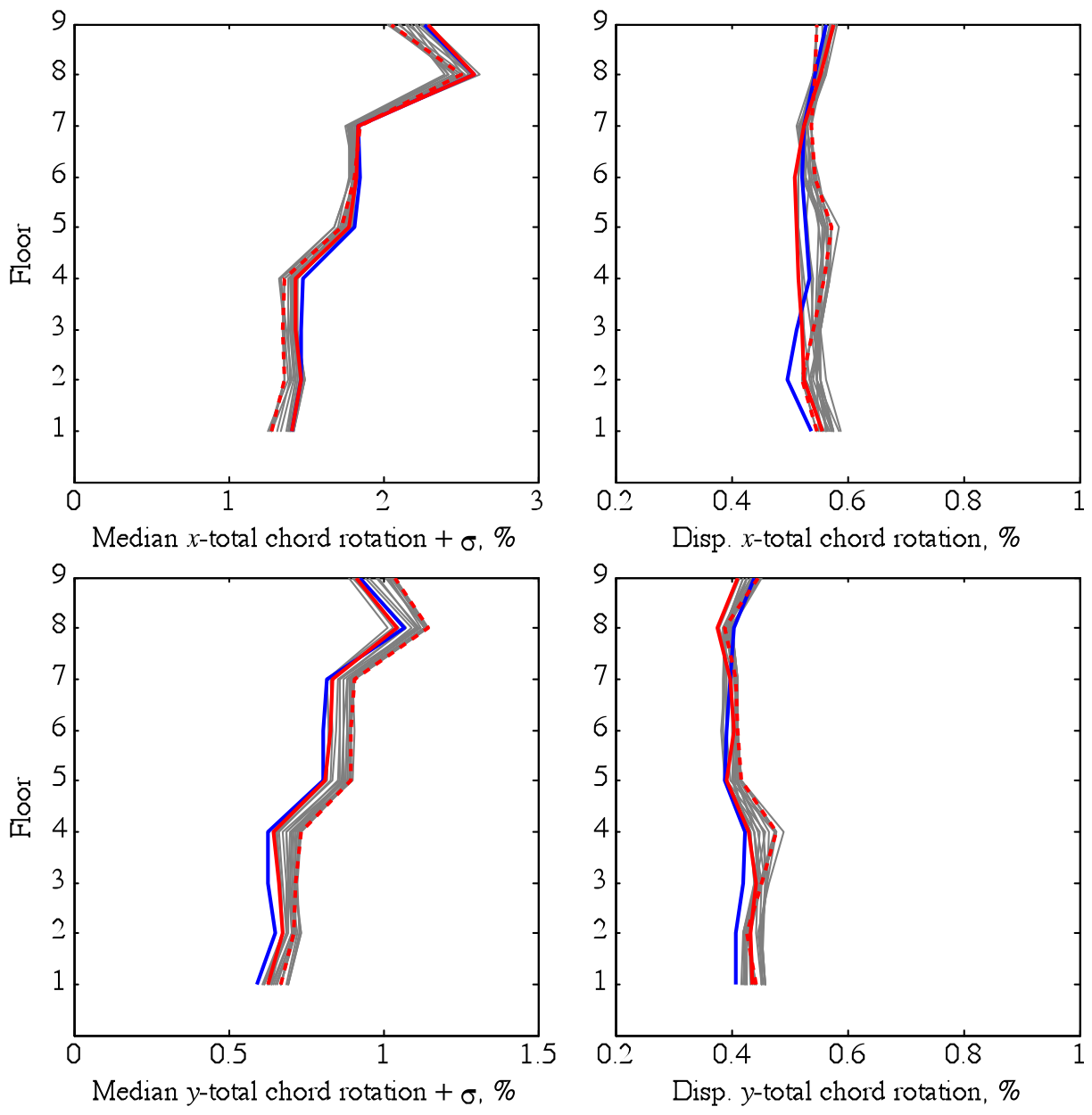

Chord rotation due to components rotated $\theta_{x}$ degrees

- -.. Chord rotation due to FN-FP components

Chord rotation due to MD components

Figure 65. Height-wise distribution of median $+\sigma$ and dispersion values of total chord rotation in the $x$ and $y$ directions for the nonlinear nine-story asymmetric-plan building. The gray, red, and blue lines show median $+\sigma$ and dispersion values of floor total accelerations due to bidirectional ground motions in arbitrary orientations in the fault-parallel and fault-normal directions (FN-FP) and in the maximum direction (MD), respectively. \%, percent; disp., displacement. 

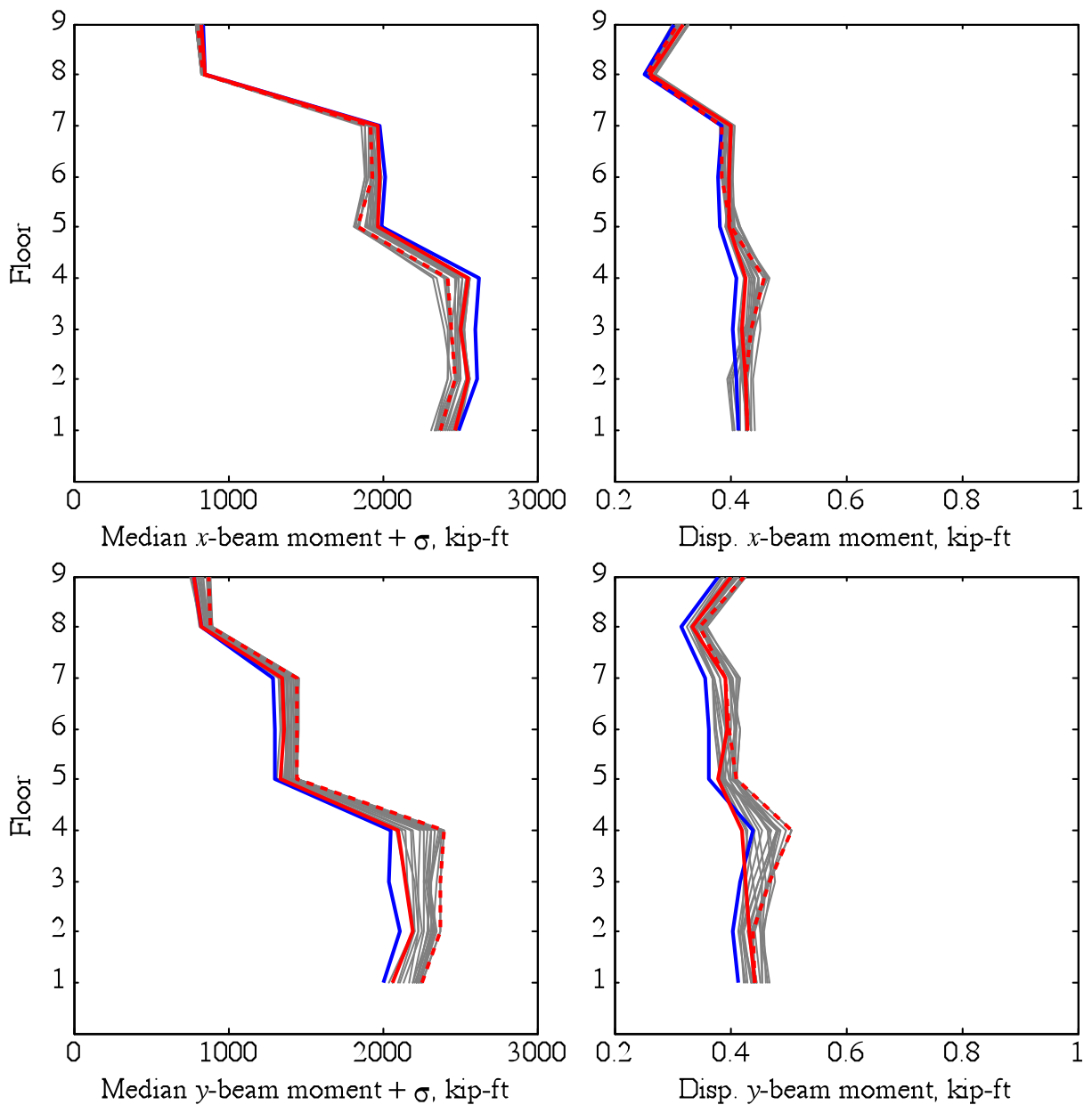

- Beam moment due to components rotated $\theta_{x}$ degrees

- -.. Beam moment due to FN-FP components

Beam moment due to MD components

Figure 66. Height-wise distribution of median $+\sigma$ and dispersion values of moments at the beams highlighted in figure 20 for the nonlinear nine-story asymmetric-plan building. The gray, red, and blue lines show median $+\sigma$ and dispersion values of beam moments due to bidirectional ground motions in arbitrary orientations in the fault-parallel and fault-normal directions (FN-FP) and in the maximum direction (MD), respectively. \%, percent; disp., displacement. 

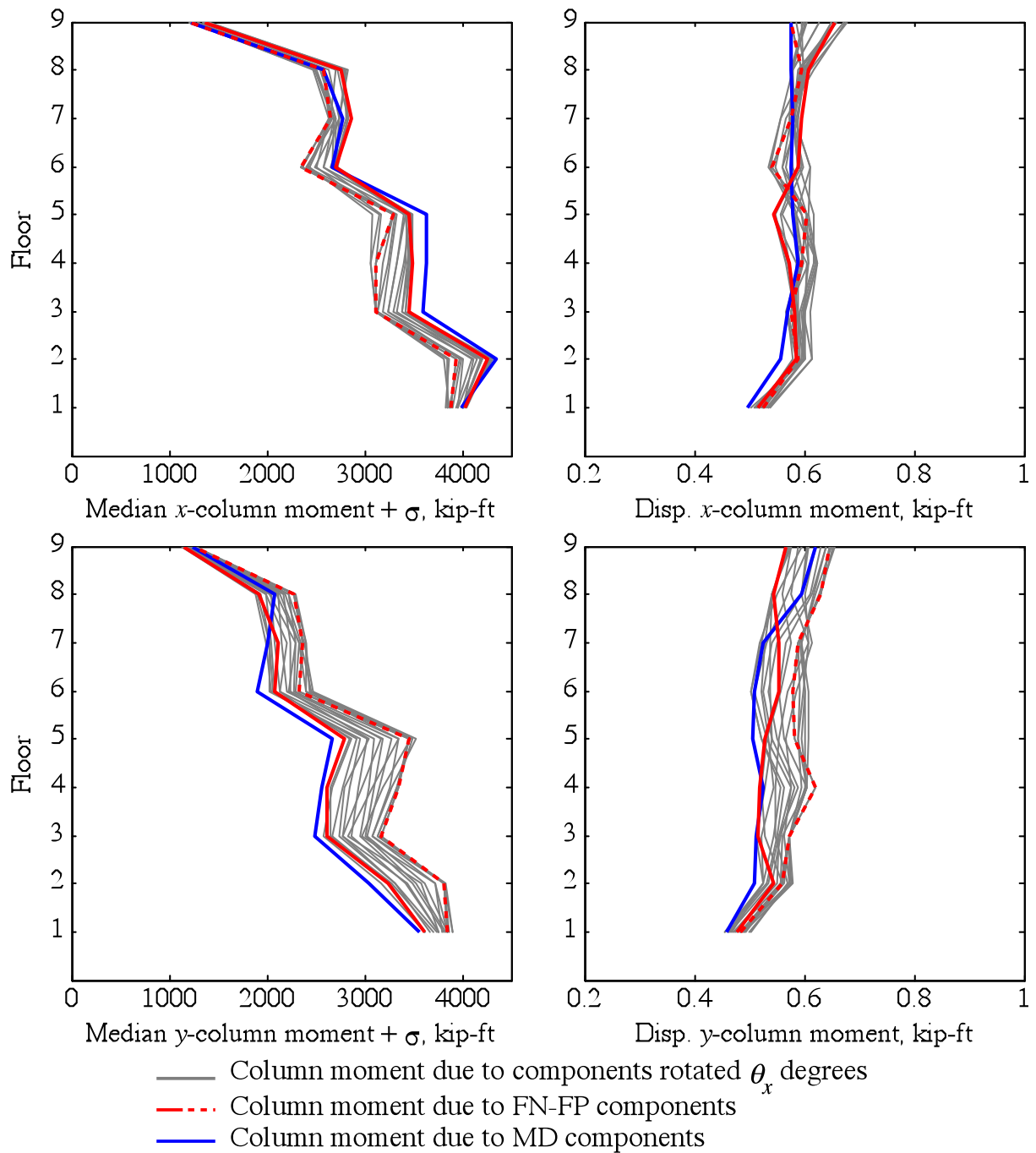

Figure 67. Height-wise distribution of median $+\sigma$ and dispersion values of moments at the columns highlighted in figure 20 for the nonlinear nine-story asymmetric-plan building. The gray, red, and blue lines show median $+\sigma$ and dispersion values of beam moments due to bidirectional ground motions in arbitrary orientations in the fault-parallel and fault-normal directions (FN-FP) and in the maximum direction (MD), respectively. \%, percent; disp., displacement. 

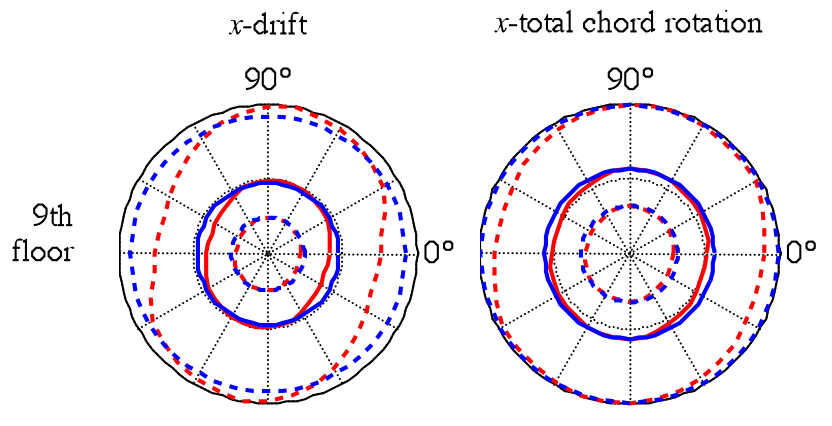

$x$-beam moment

$x$-column moment
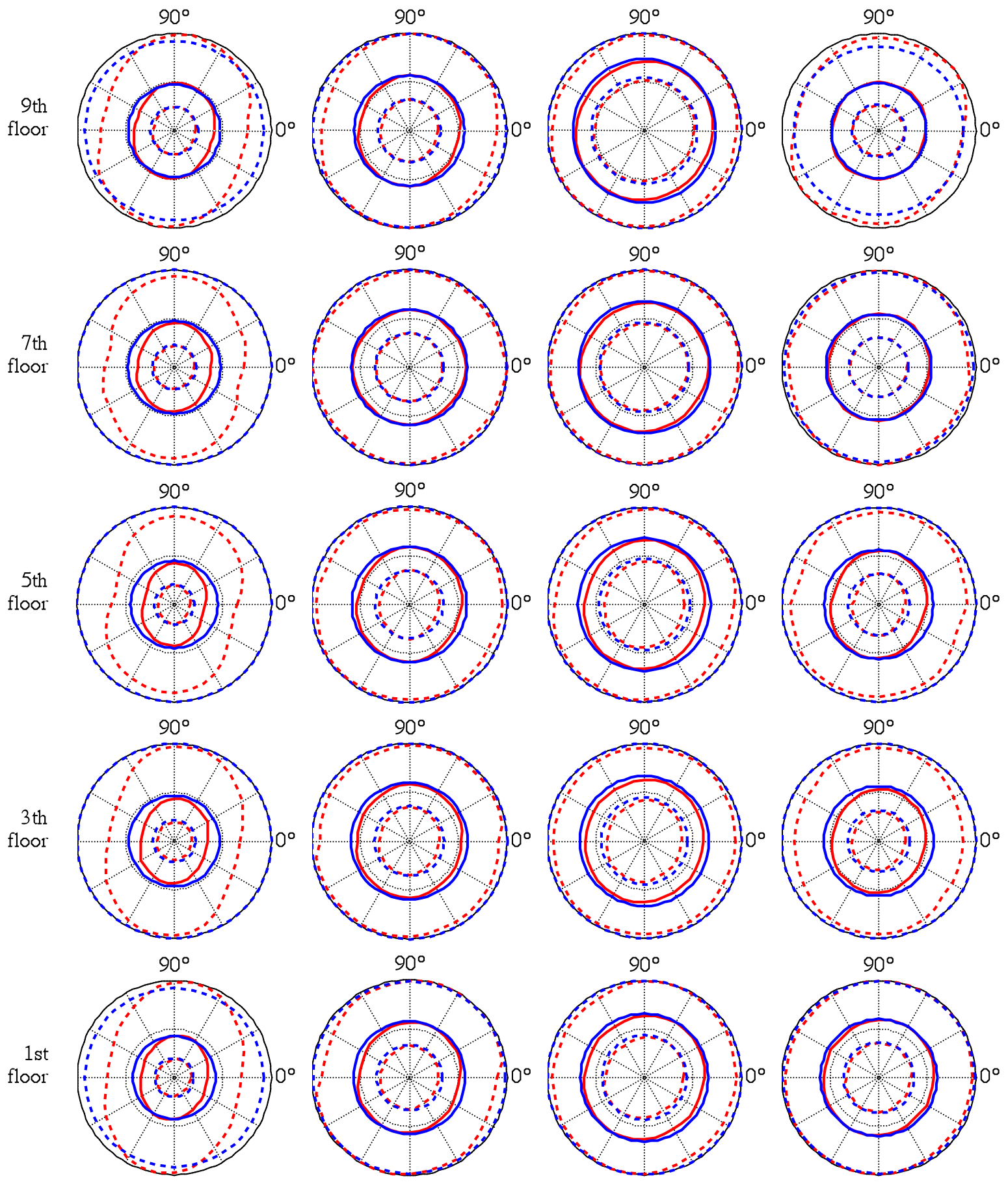

Figure 68. Median values of story drifts at the corner, total chord rotations, and internal forces in the $x$ direction as a function of the rotation angle, $\theta_{x}$, for the nonlinear nine-story asymmetric-plan building subjected to bidirectional loading. The red lines represent the median engineering demand parameter $(E D P)$ values $\pm \sigma$. The blue circles represent the median EDP values $\pm \sigma$ for the building subjected to bidirectional ground motions in the maximum direction. Solid lines are $50^{\text {th }}$ and dashed lines are $16^{\text {th }}$ and $84^{\text {th }}$ percentile EDPs. 

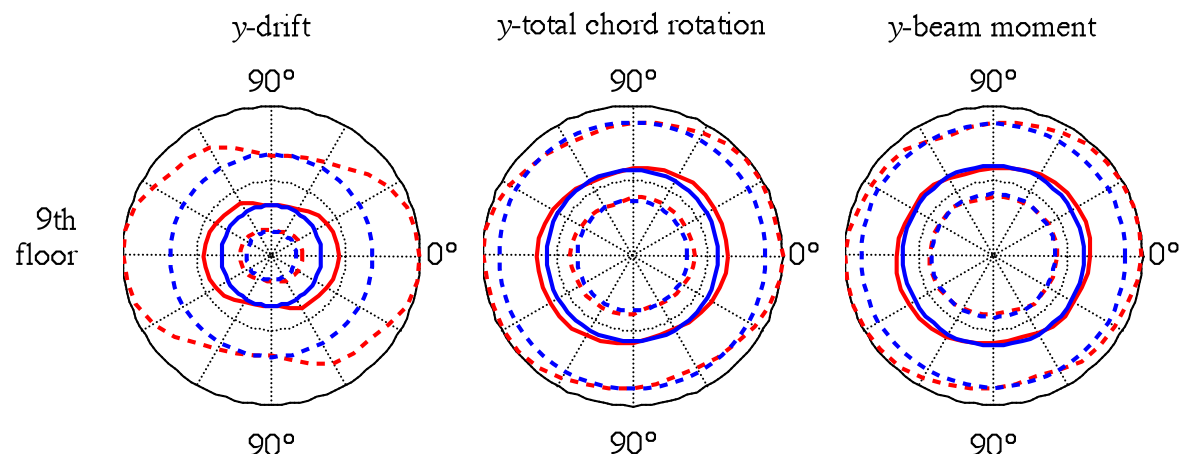

$y$-column moment
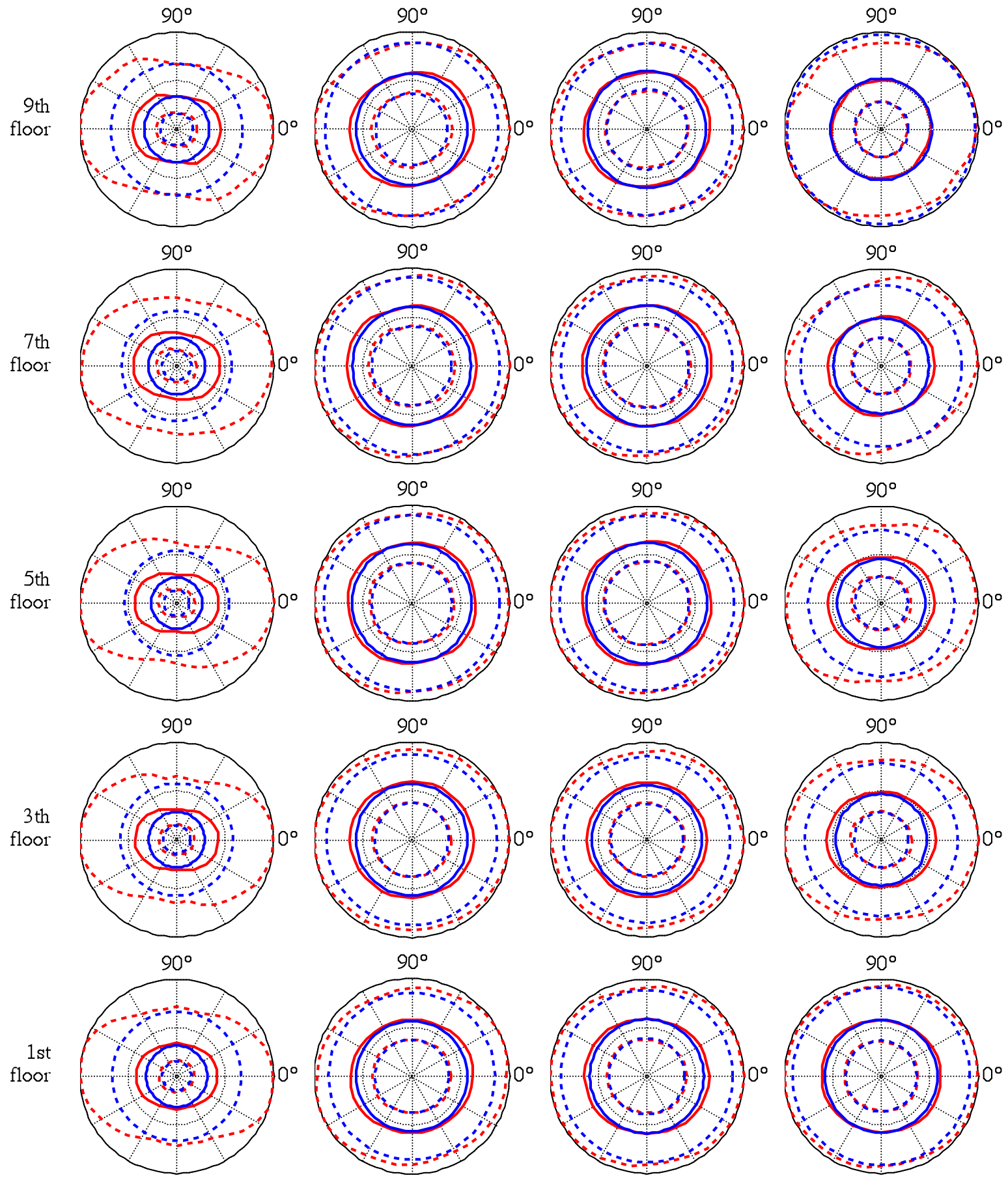

Figure 69. Median values of story drifts, total chord rotations, and internal forces in the $y$ direction as a function of the rotation angle, $\theta_{x}$, for the nonlinear nine-story asymmetric-plan building subjected to bidirectional loading. The red lines represent the median-EDP values $\pm \sigma$. The blue circles represent the median-EDP values $\pm \sigma$ for the building subjected to bidirectional ground motions in the maximum direction. Solid lines are $50^{\text {th }}$ and dashed lines are $16^{\text {th }}$ and $84^{\text {th }}$ percentile EDPs. 